\title{
Nielsen Theory of 2-valued maps on the Klein bottle
}

\author{
Bartira Maués \\ TESE APRESENTADA \\ $\mathrm{AO}$ \\ Instituto DE MATEMÁticA E EstatísticA \\ DA \\ Universidade DE SÃo PAUlo \\ PARA \\ OBTENÇÃO DO TÍTULO \\ $\mathrm{DE}$ \\ Mestre/Doutor em Ciências \\ Programa:Matemática Pura \\ Orientador: Prof. Dr. Daciberg Lima Gonçalves
}

Durante o desenvolvimento deste trabalho o autor recebeu auxílio financeiro da CAPES/CNPq/FAPESP

São Paulo, fevereiro de 2011 


\section{Nielsen Theory of 2-valued maps on the Klein bottle}

This is the original version of the thesis, as submitted to the thesis committee, written by Bartira Maués. 


\section{Resumo}

Maués, B. Teoria de Nielsen de funções à dois valores na garrafa de Klein. 2019, 44 f. Tese (Doutorado) - Instituto de Matemática e Estatística, Universidade de São Paulo, São Paulo, 2019.

Nesse trabalho estudamos funções (contínuas) à dois valores na garrafa de Klein usando teoria de tranças. Classificamos as classes de funções (à dois valores) com número de Nielsen zero em termos do grupo das tranças puras de duas cordas da garrafa de Klein. Damos condições necessárias para funções que não são split em termos do grupo total de tranças e calculamos o número de Nielsen das funções que não são split. Para variedades compactas $X$ e $Y$ estabelecemos uma conexção entre uma função à dois valores de $X$ em $Y$ e classes de funções de $\hat{X}$ em $Y$ que não tem a propriedade Borsuk-Ulam em respeito à transorfmação de Deck dada por um recobrimento específico $q: \hat{X} \rightarrow X$. Palavras-chave: funções multivaluadas, garrafa de Klein, número de Nielsen, Propriedade de Wecken, equações em grupos livres, tranças 


\section{Abstract}

\section{Maués, B. Nielsen Theory of 2-valued maps}

on the Klein bottle. 2019. 44 f. PhD Thesis - Instituto de Matemática e Estatística, Universidade de São Paulo, São Paulo, 2019.

In this work we study 2-valued maps on the Klein-bottle using braid theory. We provide a classification ofbased homotopy classes of split (2-valued) maps with Nielsen number zero in terms of the pure braid group with two strings on the Klein-bottle. We give necessary conditions, in terms of the total braid group, for maps that are non-split and compute their Nielsen number. For compact manifolds $X$ and $Y$ we establish a connection between 2-valued non-split maps from $X$ to $Y$, and classes of maps from $\hat{X}$ to $Y$ that have not the Borsuk-Ulam property in respect to the Deck trasformation given by a certain covering space $q: \hat{X} \rightarrow X$.

Keywords: multivalued maps, Klein-bottle, Nielsen number, Wecken property, equations in free groups, braids 


\section{Contents}

1 Introduction $\quad 1$

2 Braid Groups $\quad 5$

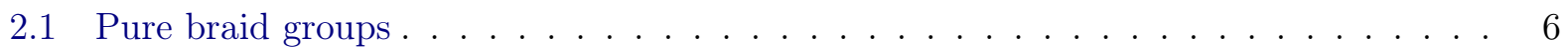

2.2 Total Braid Group $B_{2}(K) \ldots \ldots \ldots \ldots \ldots \ldots \ldots$

3 Nielsen fixed point theory on $n$-valued maps $\quad 15$

3.1 Multivalued maps and Configuration space . . . . . . . . . . . . . . . . . 15

3.2 Nielsen number of 2 -valued maps . . . . . . . . . . . . . . . . . . . . 16

3.2 .1 Split maps . . . . . . . . . . . . . . . . . . . . . . . . 16

$3.2 .2 \quad$ Non-split maps . . . . . . . . . . . . . . . . . . . . . . . . . . 17

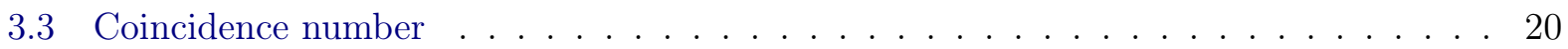

4 Homotopy classes of 2-valued split maps on the Klein bottle 23

4.1 Algebraic description and Nielsen number . . . . . . . . . . . . . . . . . 24

4.2 Solutions of an equation in the free group . . . . . . . . . . . . 30

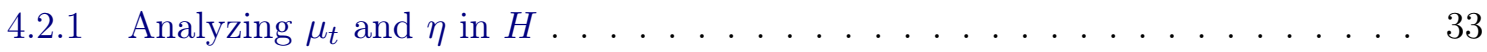

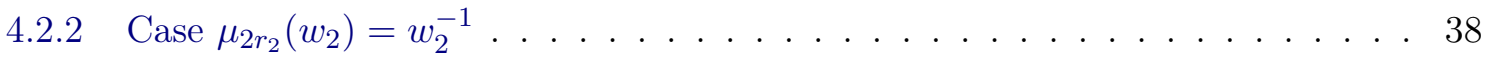

4.2 .3 Case $w_{2} \mu_{2 r_{2}+1}\left(w_{2}\right) \neq 1 \ldots \ldots \ldots \ldots \ldots \ldots \ldots$

4.3 Classification of based homotopy classes with Nielsen number zero . . . . . . . . . 57

5 Nielsen number of 2-valued non-split maps on the Klein bottle $\quad 61$

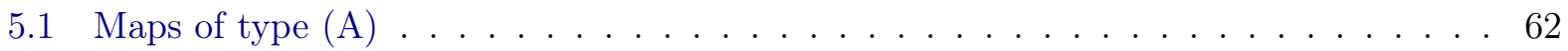

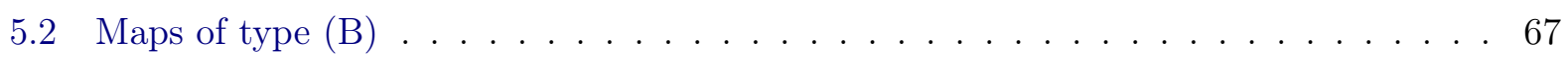

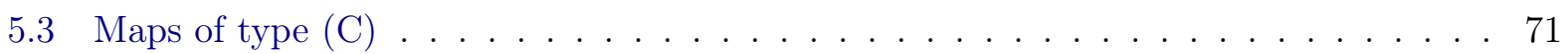

$\begin{array}{ll}\text { Bibliography } & 75\end{array}$

$\begin{array}{ll}\text { GRI Index } & 77\end{array}$ 
vi CONTENTS 


\section{Chapter 1}

\section{Introduction}

Some solutions for analytical problems can be solved by studying fixed point set of certain selfmaps $f: X \rightarrow X$, that is the set Fix $(f)=\{x \in X: f(x)=x\}$. If the Lefschetz number of a map is different to zero, then $f$ has at least one fixed point. Since the Lefschetz number of a map $f$ is defined based on the maps induced by $f$ on the homology groups it is a homotopical invariant. Hence if it is non-zero every map homotopic to $f$ has at least one fixed point. Another interesting question is how many fixed points a map homotopic to $f$ at least has. Nielsen studies this question and defines a number, now known as the Nielen number. This number is a homtopic invariant and a lower bound of the minimal number of fixed points of a map homotopic to $f$. Nielsens idea was to define certain classes of fixed points and denominate them as essential if the fixed point classes could not be removed by homotopy. A question of interest to Nielsen and that inspired this work is whether for a fixed space $X$ every selfmap $f: X \rightarrow X$ with Nielsen number zero is homotopic to a fixed point free map. This is a necessary condition for a space to be a Wecken space. The Nielsen number is the number of essential fixed point classes. A space $X$ is Wecken if for every map $f: X \rightarrow X$ there is a map $g$ homotopic to $f$ with $N(f)$ fixed points, in other words if $N(f)=M(f)$, where $M(f)=\min \{$ Fix $(g): g$ homotopic to $f\}$ is the minimal fixed point number of the class $[f]$. Wecken shows in 1942 that every triangulable compact manifold with dimension greater or equal to three is a Wecken space, see [Wec41]. At this time it is already known that the seven compact surfaces of non-negative Euler-characteristic, that is the sphere, the torus, the projective plane, the Klein bottle, the disk, the anulus and the Möbius strip, are Wecken spaces (see [Bro20] and [Hop27]). In 1984 B. Jiang shows that for every compact surface with negative Euler characteristic there is a self-map with Nielsen number equal to zero that is not deformable to a fixed point free map. That implies that a compact surface is a Wecken space if and only of its Euler characteristic is non-negative.

It is possible to ask these questions also for $n$-valued maps, which are a certain family of multivalued maps. A multivalued map $\Phi: X \rightarrow X$ is a correspondence that takes a point $x \in X$ to a non-empty set $\Phi(x) \subset Y$. An $n$-valued map is a multivalued map such that for every $x \in X$ the set $\Phi(x)=\left\{x_{1}, \ldots, x_{n}\right\}$ has cardinality $n$. The family of $n$-valued maps are specially interesting for they very similar to single valued-maps in regard to Nielsen fixed point theory. H. Schirmer defines a Nielsen number for $n$-valued maps on compact polyhedra and shows that every compact triangulable manifold (with or without boundary) with dimension at least three is a Wecken space (see [Sch85]). The projective plane has the Wecken property for $n$-valued maps (see [GG17]). In 2018 it was shown in [BCES19] that the sphere is Wecken for $n$-valued maps. There are some partial results for the torus (see [GG18]).

In this thesis we study 2-valued maps on the Klein bottle in order to give a (partial) classification, with the question in mind whether every 2 -valued map on the Klein bottle with Nielsen number zero is deformable to a fixed point free map. There is a natural correspondence between a 2 -valued maps from $X$ to $Y$ and a map from $X$ to the unordered configuration space $D_{2}(Y)$, which is the quotient $F_{2}(Y) / \mathcal{S}_{2}$ of the ordered configuration space $F_{2}(Y)=\left\{\left(y_{1}, y_{2}\right): y_{1} \neq y_{2}\right\}$ by the symmetric group 
$\mathcal{S}_{2}$ (see [GG17]). For a 2-valued map $\phi: X \multimap Y$ we denote the correspondent map $\Phi: X \rightarrow D_{2}(Y)$. If we set $X$ and $Y$ are the Klein bottle $K$, all spaces involved are $K(\pi, 1)$-spaces it follows that studying the homomorphisms between $\pi_{1}(K)$ and $\pi_{1}\left(D_{2}(K)\right)$. Now $\pi_{1}\left(D_{2}(K)\right)$ is $B_{2}(K)$, the braid group on two strands on the Klein bottle. In chapter 2 we recall a presentation of $B_{2}(K)$, the total braid group and of $P_{2}(K)$, the pure braid group and give another presentation of $B_{2}(K)$ that we use for the computations.

In chapter 3 we give a brief introduction on Nielsen theory for two valued maps between compact manifold $X$ and $Y$. A special case of (2-valued) maps are split maps, that is a map $\phi: X \rightarrow Y$ where there are maps $f_{1}, f_{2}: X \rightarrow Y$ such that $\phi(x)=\left\{f_{1}(x), f_{2}(x)\right\}$ for every $x \in X$. In this case its correspondent map $\Phi: X \rightarrow D_{2}(K)$ admits a lifting to $\Phi=\left(f_{1}, f_{2}\right): X \rightarrow F_{2}(Y)$ (see [GG18]). We denote this lift by $\hat{\Phi}$ from now on. For non-split maps we consider the subgroup $\Phi^{-1}\left(P_{2}(Y)\right)$ of $\pi_{1}(X)$, which induces a covering map $q: \hat{X} \rightarrow X$. Since this is a double covering there is a unique non-trivial deck-transformation $\delta: \hat{X} \rightarrow \hat{X}$. Then $\Phi \circ q: \hat{X} \rightarrow D_{2}(Y)$ admits a lifting $\hat{\Phi}=\left(\hat{f}_{1}, \hat{f}_{2}\right): \hat{X} \rightarrow F_{2}(Y)$, we say that $\phi$ admits a lift $\hat{\Phi}$. We show in Proposition 3.2 .5 that $\hat{f}_{2}=\hat{f}_{1} \circ \delta$ and $\hat{f}_{1}: \hat{X} \rightarrow Y$ does not have the Borsuk-Ulam property and for every pair $\left(\hat{g}_{1}, \hat{g}_{2}\right)$ that satisfies these conditions there is a 2-valued non-split map $\phi$ such that $\left(\hat{g}_{1}, \hat{g}_{2}\right)$ is the lift of $q \circ \Phi$. In [GG17] a formula for the Nielsen number for 2-valued non-split maps for orientable manifolds without boundary is given. We generalize this formula to non-orientable manifolds without boundary. This is done in Proposition 3.2.7 and uses the coincidence index for non-orientable manifolds defined in [GJ97].

In chapter 4 we study the based homotopy classes of split maps, which are in one-to-one correspondence to the homomorphisms $\pi_{1}(K) \rightarrow F_{2}(K)$. A homomorphism is defined by the image of its generators and preserves relations. It holds that if $\phi: K \multimap K$ is a 2-valued split map, then $\hat{\Phi}=\left(\hat{f}_{1}, \hat{f}_{2}\right)$ induces a homomorphism $\hat{\Phi}_{\#}: \pi_{1}(K) \rightarrow P_{2}(K)$ and for the images $\hat{\alpha}=\Phi_{\#}(\alpha)$ and $\hat{\beta}=\Phi_{\#}(\beta)$ of the generators of $\pi_{1}(K)=\left\langle\alpha \beta: \alpha \beta \alpha \beta^{-1}\right\rangle$ it holds the following equation

$$
\hat{\alpha} \hat{\beta} \hat{\alpha}=\hat{\beta} .
$$

Conversely a pair of braids $\hat{\alpha}, \hat{\beta}$ that satisfy equation (1.1) induces a map $\hat{\Phi}:\left(\hat{f}_{1}, \hat{f}_{2}\right): K \rightarrow F_{2}(K)$, that corresponds to a based homotopy class of a 2-valued map $\phi: K \rightarrow K$. Therefore we want to solve equation (1.1) in the pure braid group $P_{2}(K)$. This equation is not too easy to solve and in Proposition 4.1 .5 we narrow the pairs $(\hat{\alpha}, \hat{\beta})$ down by using that the coincidence set of $f_{1}$ and $f_{2}$ is empty, since this is a necessary condition for equation (1.1) to hold. In Theorem 4.1.6 we give an algebraic description of the homotopy classes of maps from $K$ to $F_{2}(K)$.

For a map $\phi=\left\{f_{1}, f_{2}\right\}$, that is for a pair $\hat{\alpha}, \hat{\beta} \in P_{2}(K)$ such that equation (1.1) holds, we compute the Nielsen number in terms of $\hat{\alpha}$ and $\hat{\beta}$ using the formula of H. Schirmer for $n$-valued split maps, i.e. $N(\phi)=N\left(f_{1}\right)+N\left(f_{2}\right)$. Setting the Nielsen number of $\phi$ equal to zero narrows equation (1.1) further down. We can consider the equation in each component of $F_{2} \rtimes_{\theta} \pi_{1}(K)$ and obtain an equation in the free group $F_{2}$ (see Theorem 4.1.8). By solving it we obtain a classification of 2-valued split maps with Nielsen number zero (see Theorem 4.3.1).

Chapter 5 is dedicated to study maps that are not split. As said before every 2-valued map $\phi: K \multimap K$ corresponds to a homomorphism $\Phi_{\#}: \pi_{1}(K) \rightarrow B_{2}(K)$. If $\phi$ is non-split, then $\hat{\alpha}=\Phi_{\#}(\alpha)$ and $\hat{\beta}=\Phi_{\#}(\beta)$ are not both pure braids, and be divide in three cases

(A) $\hat{\beta}$ is not pure,

(B) $\hat{\alpha}$ is not pure,

(C) both $\hat{\alpha}$ and $\hat{\beta}$ is not pure.

In each case we determine the correspondent covering space and compute in Propositions 5.1.4, 5.2.5 and 5.3.3 the Nielsen number using, for certain maps, a formula of the coincidence number given by D. Gonçalves and J. Guaschi (see Propositions 3.3.3 and 3.3.1). In case $(B)$ and $(C)$ the covering space is isomorphic to the Klein bottle and we can use the Borsuk-Ulam Property to 
determine all possible lifts which is done in Lema 5.2.3 and Proposition 5.2.4, for maps of type $(B)$ and in 5.3.1 and 5.3.2 for maps of type $(C)$. In case $(A)$ the covering space is the torus and we do not have a classification of the maps from the torus to the Klein bottle for the correspondent deck-transformation $\delta$. To narrow down the possible lifts $\hat{\Phi}=\left(\hat{f}_{1}, \hat{f}_{2}\right)$ and pairs of braids $\hat{\alpha}, \hat{\beta}$ of a map $\phi$ of type $(A)$ we use that the coincidence set of $f_{1}$ and $f_{2}$ is empty. The sum of these give us some necessary conditions for $\hat{\alpha}$ and $\hat{\beta}$ and the lift $\hat{\Phi}$.

D. Gonçalves and J. Guaschi provide an algebraic criterion involving braid groups to examine this question whether a map with Nielsen number zero is homotopic to a fixed point free map (see [GG18]). They show that, if $X$ is a compact surface without boundary and non-positive Euler characteristic, an 2-valued map $\phi: X \multimap X$ is homotopic to a fixed point free map if and only if there is a homomorphism from $\pi_{1}(X)$ to $B_{1,2}(X)$ such that the following diagram commutes up to homotopy

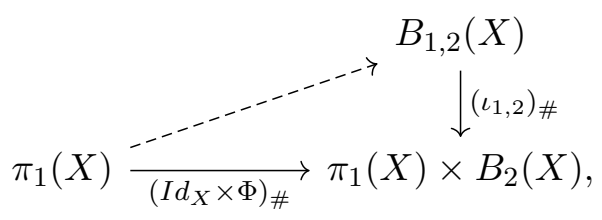

where $D_{1,2}(X)=F_{3}(X) /\{1\} \times \mathcal{S}_{2}$ is the quotient of the ordered configuration space $F_{3}(X)$ by the action of the subgroup $\{1\} \times \mathcal{S}_{2}$ of the symmetric group $\mathcal{S}_{3}$ and $\iota_{1,2}: D_{1,2}(X) \rightarrow X \times D_{2}(X)$ is the inclusion and $B_{1,2}(X)=\pi_{1}\left(D_{1,2}(X)\right)$. This holds for all 2-valued maps, but for split maps it is possible to give a criterion in terms of pure braid groups, where it is easier to make computations. If $X$ is as above, then a 2-valued split map $\phi: X \multimap X$ is homotopic to a fixed point free map if and only if there is a homomorphism $\pi_{1}(X) \rightarrow P_{3}(X)$ such that the following diagram commutes up to homotopy

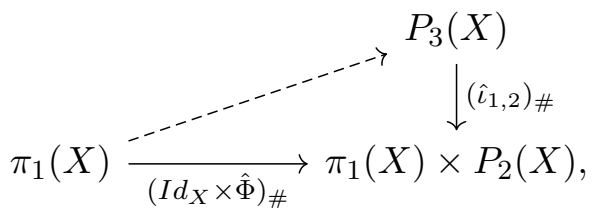

where $\hat{\iota}_{1,2}: F_{3} \rightarrow X \times F_{2}(X)$ is the inclusion map.

In this work we explicit some 2 -valued maps on the Klein bottle, which have Nielsen number equal to zero. The approach introduced above are a possible enrolment to decide whether they are deformable to fixed point free maps, which is an important part to verify if the Klein bottle is a Wecken space for 2-valued maps. Several of results obtained in this thesis are generalizable to $n$-valued maps in a quite straightforward way. Since those generalizations do not add anything substantial, we focused on 2-valued maps. 


\section{Chapter 2}

\section{Braid Groups}

For a topoogical space, the ordered configuration space of $X$ on $n$ points is defined by $F_{n}(X)=$ $\left\{\left(x_{1}, \ldots, x_{n}\right): x_{i} \neq x_{j}, i \neq j\right\}$. Considering the action of the permutation group $\mathcal{S}_{n}$ on $F_{n}(X)$ we define the unordered configuration space by $D_{n}(X)=F_{n}(X) / \mathcal{S}_{n}$. The elements in $D_{n}$ are unordered $n$-tuples and we write $\left\{x_{1}, \ldots, x_{n}\right\}$.

Fix $n$ pairwise different points $p_{1}, \ldots, p_{n}$ in $X$. The $n$-th braid group $B_{n}(X)$ is the fundamental group of the unordered configuration group $D_{n}(X)$, i.e. $B_{n}(X)=\pi_{1}\left(D_{n}(X),\left\{p_{1}, \ldots, p_{n}\right\}\right)$. The $n$-th pure braid group $P_{n}(X)$ is the fundamental group of the ordered configuration group $D_{n}(X)$, i.e. $P_{n}(X)=\pi_{1}\left(F_{n}(X),\left(p_{1}, \ldots, p_{n}\right)\right)$.

For every braid $\beta \in B_{n}(X)$ there are $n$ maps $\beta_{1}, \ldots, \beta_{n}: I \rightarrow X$ such that

$$
\beta(t)=\left\{\beta_{1}(t), \ldots, \beta_{n}(t)\right\}
$$

for every $t \in I$ and $\beta_{i}(t)=p_{i}$, where $i \in\{1, \ldots, n\}$. Consider the map that assigns a braid $\beta \in B_{n}(X)$ to a permutation $\sigma$, such that $\beta_{i}(1)=p_{\sigma(i)}$, for every $i \in\{1, \ldots, n\}$. This map naturally defines a short exact sequence.

$$
0 \longrightarrow P_{n}(X) \stackrel{i}{\longrightarrow} B_{n}(X) \stackrel{\tau}{\longrightarrow} \mathcal{S}_{n}(X) \longrightarrow 0 \text {. }
$$

Consider the homomorphism that is induced on the fundamental group by the projection $p$ : $F_{n+1}(X) \rightarrow F_{n}(X)$, which is defined by $p\left(x_{1}, \ldots, x_{n+1}\right)=\left(x_{1}, \ldots, x_{n}\right)$. Geometrically this map $p$ is the map that "forgets" the last coordinate. If $X$ is a compact surface without boundary Fadell and Neuwirth showed that this projection is a local trivial fibration in [FN62]. The fiber of a point $\left(p_{1}, \ldots, p_{n}\right)$ is isomorphic to $X \backslash\left\{p_{1}, \ldots, p_{n}\right\}$ and is considered as a subspace of $F_{n+1}(M)$ through the inclusion $i: F_{n+1}\left(X \backslash\left\{p_{1}, \ldots, p_{n}\right\}\right)$. This fibration induces the following short exact sequence

$$
0 \longrightarrow \pi_{1}\left(X \backslash\left\{p_{1}, \ldots, p_{n}\right\}\right) \stackrel{i_{\#}}{\longrightarrow} P_{n+1}(X) \stackrel{p_{\#}}{\longrightarrow} P_{n}(X) \longrightarrow 0,
$$

for $n \geq 1$ and $X$ not the sphere or the projective plane.

Given groups with the following presentation

$$
F=\langle X \mid R\rangle \text { and } H=\langle Y \mid S\rangle
$$

and a short exact sequence

$$
0 \longrightarrow F \stackrel{i}{\longrightarrow} G \stackrel{p}{\longrightarrow} H \longrightarrow 0 \text {. }
$$

it is possible to find a presentation of $G$. Set

$$
\tilde{X}=\{\tilde{x}=i(x): x \in X\}
$$


and let

$$
\tilde{R}=\{\tilde{r}=i(r): r \in R\} .
$$

be the set of words on the letters in $\tilde{X}$, where the letter $x$ in the word $r$ is replaced by $\tilde{x}=i(x)$. Let $\gamma: H \rightarrow G$ be a transversal function, i.e. $p \circ \gamma=i d_{H}$. Then set

$$
\tilde{Y}=\{\tilde{y}=\gamma(y): y \in Y\}
$$

and let $\tilde{s}$ be the words, obtained through the words $s \in S$ by replacing the letters $y$ by the letters $\tilde{y}$. For every word $s \in S$ we have that $\tilde{s}=\gamma(s) \in G$ and $p(\tilde{s})=s=1$. Therefore $\tilde{s} \in \operatorname{ker}(p)=i m(i)$ and since $i m(i)$ is generated by $\tilde{X}$, there exists a word $w_{s}$ with letters in $\tilde{X}$ such that $\tilde{s}=w_{s}$. Now set

$$
\tilde{S}=\left\{\tilde{s} w_{s}^{-1}: s \in S\right\}
$$

Note that since $\operatorname{im}(i)=\operatorname{ker}(p)$ is a normal subgroup of $G$, every conjugate $\tilde{x}^{-1} \tilde{y} \tilde{x}$ is contained in $i m(i)$ and therefore can be written as a word $w_{x, y}$ with letters in $\tilde{X}$. Set

$$
\tilde{T}=\left\{\left(\tilde{x}^{-1} \tilde{y} \tilde{x}\right) w_{x, y}^{-1}: x \in X, y \in Y\right\} .
$$

From [Joh97, Proposition 1, page 139] we have the following result.

Proposition 2.0.1. Using the notation above the group $G$ admits the following presentation:

$$
G=\langle\tilde{X}, \tilde{Y}: \tilde{R}, \tilde{S}, \tilde{T}\rangle
$$

In what follows we will denote the relators $\tilde{R}$ of type I, the relators $\tilde{S}$ of type II and the relators $\tilde{T}$ of type III.

\section{$2.1 \quad$ Pure braid groups}

We study the braid groups of the Klein bottle $K$. One presentation of the pure braid groups of $K$ is given by C. de Miranda e Pereiro.

Proposition 2.1.1. [DMeP15, Theorem 1.3.1.]

The pure braid group with $n$ strings admits the following presentation:

The set of generators is $\left\{\hat{a}_{i}, \hat{b}_{i}: i \in\{1, \ldots, n\}\right\} \cup\left\{c_{i, j}\right\}$, where

The defining relations are the following:

1. $\hat{a}_{i} \hat{a}_{j}=\hat{a}_{j} \hat{a}_{i},(1 \leq i<j \leq n)$;

2. $\hat{a}_{i}^{-1} \hat{b}_{j} \hat{a}_{i}=\hat{b}_{j} \hat{a}_{j} c_{i, j}^{-1} c_{i+1, j} \hat{a}_{j}^{-1},(1 \leq i<j \leq n)$;

3. $\hat{a}_{i}^{-1} c_{j, k} \hat{a}_{i}=\left\{\begin{array}{l}c_{j, k},(1 \leq i<j<k \leq n) \text { or }(1 \leq j<k<i \leq n), \\ \hat{a}_{k} c_{i+1, k}^{-1} c_{i, k} \hat{a}_{k}^{-1} c_{j, k} c_{i, k}^{-1} c_{i+1, k},(1 \leq j \leq i<k \leq n) ;\end{array}\right.$

4. $c_{i, l}^{-1} c_{j, k} c_{i, l}=\left\{\begin{array}{c}c_{j, k},(1 \leq i<l<j<k \leq n) \text { or }(1 \leq j \leq i<l<k \leq n), \\ c_{i, k} c_{l+1, k}^{-1} c_{l, k} c_{i, k}^{-1} c_{j, k} c_{l, k}^{-1} c_{l+1, k},(1 \leq i<j \leq l<k \leq n) ;\end{array}\right.$

5. $\prod_{j=i+1}^{n} c_{i, j} c_{i+1, j}^{-1}=\hat{b}_{i} c_{1, i} \hat{a}_{i}^{-1} \hat{b}_{i}^{-1} \hat{a}_{i}^{-1},(1 \leq i \leq n)$;

6. $\hat{b}_{i}^{-1} \hat{b}_{j} \hat{b}_{i}=\hat{b}_{j} c_{i, j} c_{i+1, j}^{-1},(1 \leq i<j \leq n)$;

7. $\hat{b}_{i}^{-1} \hat{a}_{j} \hat{b}_{i}=\hat{a}_{j} \hat{b}_{j} c_{i+1, j} c_{i, j}^{-1} \hat{b}_{j}^{-1},(1 \leq i<j \leq n)$; 
8. $\hat{b}_{i}^{-1} c_{j, k} \hat{b}_{i}=\left\{\begin{array}{l}c_{j, k},(1 \leq i<j<k \leq n) \text { or }(1 \leq j<k<i \leq n), \\ c_{i+1, k} c_{i, k}^{-1} c_{j, k} \hat{b}_{k} c_{i+1, k} c_{i, k}^{-1} \hat{b}_{k}^{-1},(1 \leq j \leq i<k \leq n)\end{array}\right.$

Here $c_{i, i}=1$ and $\prod_{j=n+1}^{n} c_{i, j} c_{i+1, j}^{-1}=1$. Note that from the identity 5 . it follows that $c_{1, n}=$ $\hat{b}_{n}^{-1} \hat{a}_{n} \hat{b}_{n} \hat{a}_{n}$, therefore $c_{1, n}$ could be eliminated from the generating set.

C. de Miranda e Pereiro also determines a section $\gamma$ for the short exact sequence (2.2).

$$
0 \longrightarrow \pi_{1}\left(K \backslash\left\{p_{1}, \ldots, p_{n}\right\}\right) \stackrel{i_{\#}}{\longrightarrow} P_{n+1}(K) \stackrel{p_{\#}}{\longrightarrow} P_{n}(K) \longrightarrow 0
$$

Proposition 2.1.2. [DMeP15, Proposition-2.2.1.] With the presentation given in 2.1.1 the following function $\gamma: P_{n}(K) \rightarrow P_{n+1}(K)$ defines a section for the short exact sequence (2.2).

$$
\begin{array}{rlr}
\gamma: P_{n}(K) & \rightarrow P_{n+1}(K) & \\
\hat{a}_{i} & \mapsto \hat{a}_{i}, & (1 \leq i<n), \\
\hat{a}_{n} & \mapsto \hat{a}_{n} \hat{a}_{n+1}, & (1 \leq i<n), \\
\hat{b}_{i} & \mapsto \hat{b}_{i}, & \\
\hat{b}_{n} & \mapsto \hat{b}_{n} \hat{b}_{n+1} c_{n, n+1}, & (1 \leq i<j \leq n-1), \\
c_{i, j} & \mapsto c_{i, j}, & (1 \leq i<n) . \\
c_{i, n} & \mapsto c_{i, n} c_{i, n+1} c_{n, n+1}^{-1}, &
\end{array}
$$

Therefore $P_{n+1}(K)$ can be written as a semi-direct product of $\pi_{1}\left(K \backslash\left\{p_{1}, \ldots, p_{n}\right\}\right)$ and $P_{n}(K)$.

\section{The pure braid group on two strings $P_{2}(K)$}

Setting $n=1$ the fundamental group of $K \backslash\left\{p_{1}\right\}$ is the fundamental group of the figure eight, which is $F_{2}\left(a_{2}, b_{2}\right)$ or $F_{2}$, the free group on two letters $a_{2}$ and $b_{2}$. Also $P_{i}(K)=\pi_{1}(K)=\left\langle a_{1}, b_{1}\right.$ : $\left.b_{1}^{-1} a_{1} b_{1} a_{1}\right\rangle$, hence the short exact sequence (2.2) is as follows:

$$
0 \longrightarrow F_{2} \stackrel{i_{\#}}{\longrightarrow} P_{2}(K) \underset{K_{-} \ldots-}{\stackrel{p_{\#}}{\longrightarrow}} \pi_{1}(K) \longrightarrow 0
$$

where $\gamma$ is the section given in Proposition 2.1.2. Using the identities of Proposition 2.1.1 the section $\gamma$ is given by

$$
\begin{aligned}
\gamma: \pi_{1}(K) & \longrightarrow P_{2}(K) \\
a_{1} & \longmapsto \hat{a}_{1} \hat{a}_{2}=\hat{a}_{2} \hat{a}_{1} \\
b_{1} & \longmapsto \hat{b}_{1} \hat{b}_{2} c_{1,2}=\hat{b}_{2} \hat{b}_{1}
\end{aligned}
$$

From Proposition 2.1.1 we know that pure braid group with 2 strings admits the following presentation:

The generating set is $\left\{\hat{a}_{1}, \hat{b}_{1}, \hat{a}_{2}, \hat{b}_{2}\right\}$ and the relations are
(a) $\hat{a}_{1} \hat{a}_{2}=\hat{a}_{2} \hat{a}_{1}$,
(b) $\hat{a}_{1}^{-1} \hat{b}_{2} \hat{a}_{1}=\hat{a}_{2}^{-1} \hat{b}_{2} \hat{a}_{2}^{-1}$,
(c) $\hat{b}_{1}^{-1} \hat{a}_{2} \hat{b}_{1}=\hat{a}_{2} \hat{b}_{2} \hat{a}_{2}^{-1} \hat{b}_{2}^{-1} \hat{a}_{2}^{-1}$,
(d) $\hat{b}_{1}^{-1} \hat{b}_{2} \hat{b}_{1}=\hat{a}_{2} \hat{b}_{2} \hat{a}_{2}$,
(e) $\hat{b}_{1} \hat{a}_{1}^{-1} \hat{b}_{1}^{-1} \hat{a}_{1}^{-1}=\hat{b}_{2}^{-1} \hat{a}_{2} \hat{b}_{2} \hat{a}_{2}$. 
The following relations, which are useful for our computations, hold in $P_{2}(K)$ as well.

Observation 2.1.3. In $P_{2}(K)$ the following holds:

(i) $\hat{a}_{1} \hat{b}_{2} \hat{a}_{1}^{-1}=\hat{a}_{2} \hat{b}_{2} \hat{a}_{2}$.

In fact from relation (b) it follows

$$
\hat{b}_{2}=\hat{a}_{1} \hat{a}_{2}^{-1} \hat{b}_{2} \hat{a}_{2}^{-1} \hat{a}_{1}^{-1}=\hat{a}_{2}^{-1} \hat{a}_{1} \hat{b}_{2} \hat{a}_{1}^{-1} \hat{a}_{2}^{-1} .
$$

(ii) $\hat{b}_{1} \hat{a}_{2} \hat{b}_{1}^{-1}=\hat{b}_{2}^{-1} \hat{a}_{2}^{-1} \hat{b}_{2}$.

From relation (d) it follows that

$$
\hat{b}_{2}=\hat{b}_{1} \hat{a}_{2} \hat{b}_{2} \hat{a}_{2} \hat{b}_{1}^{-1}
$$

Thus equation (c) implies

$$
\hat{a}_{2}^{-1}=\hat{b}_{1} \hat{a}_{2} \hat{b}_{2} \hat{a}_{2} \hat{b}_{2}^{-1} \hat{a}_{2}^{-1} \hat{b}_{1}^{-1}=\left(\hat{b}_{1} \hat{a}_{2} \hat{b}_{2} \hat{a}_{2} \hat{b}_{1}^{-1}\right)\left(\hat{b}_{1} \hat{a}_{2} \hat{b}_{1}^{-1}\right)\left(\hat{b}_{1} \hat{a}_{2}^{-1} \hat{b}_{2}^{-1} \hat{a}_{2}^{-1} \hat{b}_{1}^{-1}\right)=\hat{b}_{2}\left(\hat{b}_{1} \hat{a}_{2} \hat{b}_{1}^{-1}\right) \hat{b}_{2}^{-1} .
$$

(iii) $\hat{b}_{1} \hat{b}_{2} \hat{b}_{1}^{-1}=\hat{b}_{2}^{-1} \hat{a}_{2} \hat{b}_{2} \hat{a}_{2} \hat{b}_{2}$.

Since, from relation (c) it follows

$$
\hat{b}_{2}=\hat{b}_{1} \hat{a}_{2} \hat{b}_{2} \hat{a}_{2} \hat{b}_{1}^{-1}=\left(\hat{b}_{2}^{-1} \hat{a}_{2}^{-1} \hat{b}_{2}\right)\left(\hat{b}_{1} \hat{b}_{2} \hat{b}_{1}^{-1}\right)\left(\hat{b}_{2}^{-1} \hat{a}_{2}^{-1} \hat{b}_{2}\right) .
$$

Therefore

$$
\hat{b}_{1} \hat{b}_{2} \hat{b}_{1}^{-1}=\left(\hat{b}_{2}^{-1} \hat{a}_{2} \hat{b}_{2}\right) \hat{b}_{2}\left(\hat{b}_{2}^{-1} \hat{a}_{2} \hat{b}_{2}\right)=\hat{b}_{2}^{-1} \hat{a}_{2} \hat{b}_{2} \hat{a}_{2} \hat{b}_{2} .
$$

Corollary 2.1.3.1. $P_{2}(K)$ is isomorphic to $F_{2} \rtimes_{\theta_{1}} \pi_{1}(K)$, where the section $\gamma$ from the short exact sequence (2.3) induces the homomorphism $\theta_{1}: \pi_{1}(K) \rightarrow \operatorname{Aut}\left(F_{2}\right)$ that is given by

$$
\begin{aligned}
\theta_{1}\left(a_{1}\right): F_{2} & \rightarrow F_{2} \\
a_{2} & \mapsto a_{2} \\
b_{2} & \mapsto a_{2}^{2} b_{2}
\end{aligned}
$$

and

$$
\begin{aligned}
\theta_{1}\left(b_{1}\right): F_{2} & \rightarrow F_{2} \\
a_{2} & \mapsto a_{2}^{-1} \\
b_{2} & \mapsto a_{2} b_{2} a_{2} .
\end{aligned}
$$

The isomorphism is given by

$$
\begin{aligned}
\Gamma: F_{2} \rtimes_{\theta_{1}} \pi_{1}(K) & \longrightarrow P_{2}(K) \\
a_{1} & \longmapsto \hat{a}_{2} \hat{a}_{1} \\
b_{1} & \longmapsto \hat{b}_{2} \hat{b}_{1} \\
a_{2} & \longmapsto \hat{a}_{2} \\
b_{2} & \longmapsto \hat{b}_{2} .
\end{aligned}
$$


Proof. The homomorphism $\theta_{1}: \pi_{1}(K) \rightarrow \operatorname{Aut}\left(F_{2}\right)$ is given through the section $\gamma$ by

$$
\begin{array}{r}
\theta_{1}\left(a_{1}\right)\left(a_{2}\right)=i_{\#}^{-1}\left(\gamma\left(a_{1}\right) i_{\#}\left(a_{2}\right) \gamma\left(a_{1}^{-1}\right)\right)=i_{\#}^{-1}\left(\hat{a}_{2} \hat{a}_{1} \hat{a}_{2} \hat{a}_{1}^{-1} \hat{a}_{2}^{-1}\right)=i_{\#}^{-1}\left(\hat{a}_{2}\right)=a_{2} \\
\theta_{1}\left(a_{1}\right)\left(b_{2}\right)=i_{\#}^{-1}\left(\hat{a}_{2} \hat{a}_{1} \hat{b}_{2} \hat{a}_{1}^{-1} \hat{a}_{2}^{-1}\right)=i_{\#}^{-1}\left(\hat{a}_{2} \hat{a}_{2} \hat{b}_{2} \hat{a}_{2} \hat{a}_{2}^{-1}\right)=i_{\#}^{-1}\left(\hat{a}_{2}^{2} \hat{b}_{2}\right)=a_{2}^{2} b_{2} \\
\theta_{1}\left(b_{1}\right)\left(a_{2}\right)=i_{\#}^{-1}\left(\hat{b}_{2} \hat{b}_{1} \hat{a}_{2} \hat{b}_{1}^{-1} \hat{b}_{2}^{-1}\right)=i_{\#}^{-1}\left(\hat{b}_{2} \hat{b}_{2}^{-1} \hat{a}_{2}^{-1} \hat{b}_{2} \hat{b}_{2}^{-1}\right)=a_{2}^{-1} \\
\theta_{1}\left(b_{1}\right)\left(b_{2}\right)=i_{\#}^{-1}\left(\hat{b}_{2} \hat{b}_{1} \hat{b}_{2} \hat{b}_{1}^{-1} \hat{b}_{2}^{-1}\right)=i_{\#}^{-1}\left(\hat{b}_{2} \hat{b}_{2}^{-1} \hat{a}_{2} \hat{b}_{2} \hat{a}_{2} \hat{b}_{2} \hat{b}_{2}^{-1}\right)=i_{\#}^{-1}\left(\hat{a}_{2} \hat{b}_{2} \hat{a}_{2}\right)=a_{2} b_{2} a_{2} .
\end{array}
$$

Here we used the presentation given in Proposition 2.1.1 and the identities computed in observation 2.1.3. The isomorphism is given by

$$
\begin{aligned}
\Gamma: F_{2} \rtimes_{\theta_{1}} \pi_{1}(K) & \longrightarrow P_{2}(K) \\
a_{1} & \longmapsto \gamma\left(a_{1}\right)=\hat{a}_{2} \hat{a}_{1} \\
b_{1} & \longmapsto \gamma\left(b_{1}\right)=\hat{b}_{2} \hat{b}_{1} \\
a_{2} & \longmapsto i_{\#}\left(a_{2}\right)=\hat{a}_{2} \\
b_{2} & \longmapsto i_{\#}\left(b_{2}\right)=\hat{b}_{2} .
\end{aligned}
$$

Recall that an element in the semi-direct product is written by a pair. We say that a pair $(w, x) \in F_{2} \rtimes_{\theta_{1}} \pi_{1}(K)$ is in its normal form, if $w \in F_{2}$ and $x=a_{1}^{r} b_{1}^{s}$ is given in $\pi_{1}(K)$ in normal form.

Lemma 2.1.4. It holds for every $r, s \in \mathbb{Z}$ that

$$
\begin{aligned}
\theta_{1}\left(a_{1}^{r} b_{1}^{s}\right): F_{2} & \longrightarrow F_{2} \\
a_{2} & \longmapsto a_{2}^{(-1)^{s}}, \\
b_{2} & \longmapsto a_{2}^{2 r+\frac{1}{2}\left(1-(-1)^{s}\right)} b_{2} a_{2}^{\frac{1}{2}\left(1-(-1)^{s}\right)} .
\end{aligned}
$$

Proof. First note that $\theta_{1}\left(b_{1}^{2}\right)=i d_{F_{2}}$. In fact

$$
\begin{array}{r}
\theta_{1}\left(b_{1}^{2}\right)\left(a_{2}\right)=\theta_{1}\left(b_{1}\right)\left(a_{2}^{-1}\right)=a_{2} \\
\theta_{1}\left(b_{1}^{2}\right)\left(b_{2}\right)=\theta_{1}\left(b_{1}\right)\left(a_{2} b_{2} a_{2}\right)=a_{2}^{-1} a_{2} b_{2} a_{2} a_{2}^{-1}=b_{2} .
\end{array}
$$

Also, since $b_{1}^{2} a_{1}=a_{1} b_{1}^{2}$, it holds that $b_{1}^{2}$ is in the center of $F_{2} \rtimes_{\theta_{1}} \pi_{1}(K)$. Hence, for every $s \in \mathbb{Z}$, it holds $\theta_{1}\left(a_{1}^{r} b_{1}^{2 s}\right)=\theta_{1}\left(a_{1}^{r}\right)$. Inductively we can show that $\theta_{1}\left(a_{1}^{r}\right)\left(b_{2}\right)=a_{2}^{2 r} b_{2}$, therefore it holds for every $r, s \in \mathbb{Z}$

$$
\begin{aligned}
\theta_{1}\left(a_{1}^{r} b_{1}^{2 s}\right): F_{2} & \longrightarrow F_{2} \\
a_{2} & \longmapsto a_{2}, \\
b_{2} & \longmapsto a_{2}^{2 r} b_{2} .
\end{aligned}
$$

As well as for every $r, s \in \mathbb{Z}$ holds

$$
\theta_{1}\left(a_{1}^{r} b_{1}^{2 s+1}\right)=\theta_{1}\left(a_{1}^{r} b_{1}\right) \theta_{1}\left(b_{1}^{2} s\right)=\theta_{1}\left(a_{1}^{r} b_{1}\right) .
$$

From

$$
\begin{aligned}
& \theta_{1}\left(a_{1}^{r} b_{1}\right)\left(a_{2}\right)=\theta_{1}\left(a_{1}^{r}\right)\left(a_{2}^{-1}\right)=a_{2}^{-1} \\
& \theta_{1}\left(a_{1}^{r} b_{1}\right)\left(b_{2}\right)=\theta_{1}\left(a_{1}^{r}\right)\left(a_{2} b_{2} a_{2}\right)=a_{2} a_{2}^{2 r} b_{2} a_{2}=a_{2}^{2 r+1} b_{2} a_{2} .
\end{aligned}
$$


it follows for every $r, s \in \mathbb{Z}$ that

$$
\begin{aligned}
\theta_{1}\left(a_{1}^{r} b_{1}^{2 s+1}\right): F_{2} & \longrightarrow F_{2} \\
a_{2} & \longmapsto a_{2}^{-1}, \\
b_{2} & \longmapsto a_{2}^{2 r+1} b_{2} a_{2} .
\end{aligned}
$$

Observation 2.1.5. The homomorphisms $\theta_{1}\left(a_{1}\right)$ and $\theta_{1}\left(b_{1}\right)$ take the normal subgroup $\left\langle\left\langle b_{2}^{-1} a_{2} b_{2} a_{2}\right\rangle\right\rangle$ to itself, i.e; $\theta_{1}\left(a_{1}\right)\left(\left\langle\left\langle b_{2}^{-1} a_{2} b_{2} a_{2}\right\rangle\right\rangle\right) \subseteq\left\langle\left\langle b_{2}^{-1} a_{2} b_{2} a_{2}\right\rangle\right\rangle$ and $\theta_{1}\left(b_{1}\right)\left(\left\langle\left\langle b_{2}^{-1} a_{2} b_{2} a_{2}\right\rangle\right\rangle\right) \subseteq\left\langle\left\langle b_{2}^{-1} a_{2} b_{2} a_{2}\right\rangle\right\rangle$. Indeed

$$
\begin{aligned}
& \theta_{1}\left(a_{1}\right)\left(b_{2}^{-1} a_{2} b_{2} a_{2}\right)=b_{2}^{-1} a_{2}^{-2} a_{2} a_{2}^{2} b_{2} a_{2}=\left(b_{2}^{-1} a_{2} b_{2} a_{2}\right), \\
& \theta_{1}\left(b_{1}\right)\left(b_{2}^{-1} a_{2} b_{2} a_{2}\right)=a_{2}^{-1} b_{2}^{-1} a_{2}^{-1} a_{2}^{-1} a_{2} b_{2} a_{2} a_{2}^{-1}=\left(b_{2}^{-1} a_{2} b_{2} a_{2}\right)^{-1} .
\end{aligned}
$$

Note that $\theta_{1}\left(a_{1}\right)$ and $\theta_{1}\left(b_{1}\right)$ induce homomorphisms on the quotient

$$
\pi_{1}(K)=F_{2} /\left\langle\left\langle b_{2}^{-1} a_{2} b_{2} a_{2}\right\rangle\right\rangle .
$$

Therefore, the following diagram commutes,

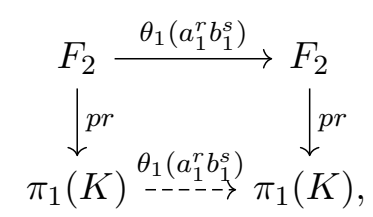

where $p r: F_{2} \rightarrow F_{2} /\left\langle\left\langle b_{2}^{-1} a_{2} b_{2} a_{2}\right\rangle\right\rangle=\pi_{1}(K)$ is the projection. It holds that

$$
\begin{aligned}
\theta_{1}\left(a_{1}^{r} b_{1}^{s}\right): \pi_{1}(K) & \longrightarrow \pi_{1}(K) \\
a_{2} & \longmapsto a_{2}^{(-1)^{s}} \\
b_{2} & \longmapsto a_{2}^{2 r} b_{2} .
\end{aligned}
$$

\subsection{Total Braid Group $B_{2}(K)$}

For the total braid group consider the presentation in the following Theorem.

Theorem 2.2.1. [DMeP15, Theorem 1.4.1] The $n$-th total braid group admits the following presentation.

The generating set is $\left\{a, b, \sigma_{1}, \ldots, \sigma_{n-1}\right\}$, and the defining relations are

1. $\sigma_{i} \sigma_{i+1} \sigma_{i}=\sigma_{i+1} \sigma_{i} \sigma_{i+1}(1 \leq i<n)$;

2. $\sigma_{j} \sigma_{i}=\sigma_{i} \sigma_{j},(2 \leq|i-j|)$;

3. $a \sigma_{j}=\sigma_{j} a,(2 \leq j)$;

4. $b \sigma_{j}=\sigma_{j} b,(2 \leq j)$;

5. $b^{-1} \sigma_{1} a=\sigma_{1} a \sigma_{1} b^{-1} \sigma_{1}$;

6. $a\left(\sigma_{1} a \sigma_{1}\right)=\left(\sigma_{1} a \sigma_{1}\right) a$;

7. $b\left(\sigma_{1}^{-1} b \sigma_{1}\right)=\left(\sigma_{1}^{-1} b \sigma_{1}^{-1}\right) b$;

8. $\sigma_{1} \sigma_{2} \cdot \ldots \cdot \sigma_{n-1}^{2} \cdot \ldots \cdot \sigma_{2} \sigma_{1}=b a^{-1} b^{-1} a^{-1}$ 
The generators of the subgroup $P_{2}(K)$, which are $a_{1}, b_{1}, a_{2}$ and $b_{2}$ are given, in this presentation for $B_{2}(K)$, by $a, b, \sigma_{1} a \sigma_{1}$ and $\sigma_{1}^{-1} b \sigma_{1}^{-1}$, respectively. In this work we work with another presentation, where the semi-direct product $F_{2} \rtimes_{\theta_{1}} \pi_{1}(K)$ appears. Consider the short exact sequence (2.1), given by the natural map to the symmetric group with $n=2$,

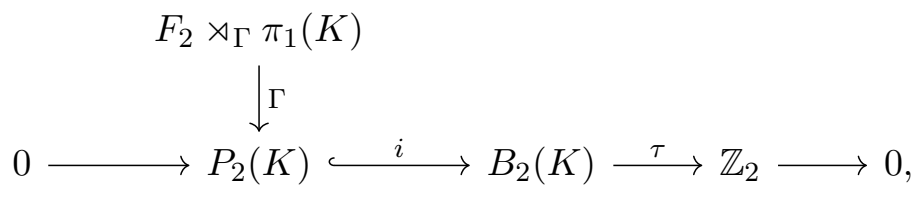

where $\tau$ is the map given in 2.1, that is the kernel of $\tau$ are the pure braids. Also $\Gamma$ is the isomorphism given by Corollary 2.1.3.1. Then $(i \circ \Gamma)$ is given by

$$
\begin{aligned}
(i \circ \Gamma): F_{2} \rtimes_{\theta_{1}} \pi_{1}(K) & \longrightarrow B_{2}(K) \\
a_{1} & \longmapsto \sigma_{1} a \sigma_{1} a \\
b_{1} & \longmapsto \sigma_{1}^{-1} b \sigma_{1}^{-1} b \\
a_{2} & \longmapsto \sigma_{1} a \sigma_{1} \\
b_{2} & \longmapsto \sigma_{1}^{-1} b \sigma_{1}^{-1} .
\end{aligned}
$$

Then $B_{2}(K)$ admits the following presentation:

Theorem 2.2.2. The braid group with two strings admits the following presentation.

The generating set is $\left\{a_{1}, b_{1}, a_{2}, b_{2}, \sigma\right\}$ and the defining relations are

1. $a_{1} a_{2}=a_{2} a_{1}$;

2. $a_{1} b_{2} a_{1}^{-1}=a_{2}^{2} b_{2}$;

3. $b_{1} a_{2} b_{1}^{-1}=a_{2}^{-1}$;

4. $b_{1} b_{2} b_{1}^{-1}=a_{2} b_{2} a_{2}$;

5. $a_{1} b_{1} a_{1} b_{1}^{-1}=1$;

6. $\sigma^{2}=b_{2}^{-1} a_{2} b_{2} a_{2}$;

7. $\sigma a_{1} \sigma^{-1}=a_{1}$;

8. $\sigma b_{1} \sigma^{-1}=b_{2}^{-1} a_{2} b_{2} a_{2} b_{1}$;

9. $\sigma a_{2} \sigma^{-1}=b_{2}^{-1} a_{2} b_{2} a_{1}$;

10. $\sigma b_{2} \sigma^{-1}=b_{2}^{-2} a_{2} b_{2} a_{2} b_{1}$;

Proof. Using the short exact sequence

$$
0 \longrightarrow F_{2} \rtimes_{\theta_{1}} \pi_{1}(K) \stackrel{(i \circ \Gamma)}{\longrightarrow} B_{2}(K) \stackrel{\tau}{\longrightarrow} \mathbb{Z}_{2} \longrightarrow 0,
$$

and Proposition 2.0.1 it follows that the generating set for $B_{2}(K)$ is $\left\{(i \circ \Gamma)\left(a_{1}\right),(i \circ \Gamma)\left(b_{1}\right),(i \circ\right.$ $\left.\Gamma)\left(a_{2}\right),(i \circ \Gamma)\left(b_{2}\right), \sigma\right\}$, where $\sigma$ is a transversal of $\tau$. Since $\pi(\sigma)=1$ we can choose $\sigma$ being $\sigma_{1}$. The first five relations are of type I, given by the relations in $F_{2} \rtimes_{\theta_{1}} \pi_{1}(K)$, see Corollary 2.1.3.1.

There is one defining relation in $\mathbb{Z}_{2}$ hence there is only one relation of type II. From relation 8. in 2.2.1 it follows that $b^{-1} \sigma^{2} a b a=1$, hence

$$
\sigma^{2}=\sigma b^{-1} \sigma^{2} a b a \sigma=\left(\sigma b^{-1} \sigma\right)(\sigma a \sigma)\left(\sigma^{-1} b \sigma^{-1}\right)(\sigma a \sigma)=(i \circ \Gamma)\left(b_{2}^{-1} a_{2} b_{2} a_{2}\right) .
$$


The relations 7 . to 10 . are of type III and are computed by conjugating the generators of $F_{2} \rtimes_{\theta_{1}} \pi_{1}(K)$. Here we use the relations of Theorem 2.2.1, as well as the relations of type I and II.

$$
\begin{aligned}
\sigma(i \circ \Gamma)\left(a_{1}\right) \sigma^{-1} & =\sigma(\sigma a \sigma a) \sigma^{-1}=\sigma(a \sigma a \sigma) \sigma^{-1}=\sigma a \sigma a=(i \circ \Gamma)\left(a_{1}\right), \\
\sigma(i \circ \Gamma)\left(b_{1}\right) \sigma^{-1} & =\sigma\left(\sigma^{-1} b \sigma^{-1} b\right) \sigma^{-1}=\sigma\left(b \sigma^{-1} b \sigma\right) \sigma^{-1}=\sigma b \sigma^{-1} b=\sigma^{2}\left(\sigma^{-1} b \sigma^{-1} b\right)=(i \circ \Gamma)\left(b_{2}^{-1} a_{2} b_{2} a_{2}\right), \\
\sigma(i \circ \Gamma)\left(a_{2}\right) \sigma^{-1} & =\sigma(\sigma a \sigma) \sigma^{-1}=\sigma^{2} a=\sigma^{2}(\sigma a \sigma)^{-1}(\sigma a \sigma a)=(i \circ \Gamma)\left(\left(b_{2}^{-1} a_{2} b_{2} a_{2}\right) a_{2}^{-1} a_{1}\right) \\
& =(i \circ \Gamma)\left(b_{2}^{-1} a_{2} b_{2} a_{1}\right), \\
\sigma(i \circ \Gamma)\left(b_{2}\right) \sigma^{-1} & =\sigma\left(\sigma^{-1} b \sigma^{-1}\right) \sigma^{-1}=b \sigma^{-2}=\left(\sigma^{-1} b \sigma^{-1}\right)^{-1}\left(\sigma^{-1} b \sigma^{-1} b\right) \sigma^{-2} \\
& =(i \circ \Gamma)\left(b_{2}^{-1} b_{1}\left(a_{2}^{-1} b_{2}^{-1} a_{2}^{-1} b_{2}\right)\right)=(i \circ \Gamma)\left(b_{2}^{-1}\left(a_{2}\left(a_{2}^{-1} b_{2}^{-1} a_{2}^{-1}\right) a_{2}\left(a_{2} b_{2} a_{2}\right)\right) b_{1}\right) \\
& =(i \circ \Gamma)\left(b_{2}^{-2} a_{2} b_{2} a_{2} b_{1}\right) .
\end{aligned}
$$

We identify $a_{1}, b_{1}, a_{2}$ and $b_{2}$ with $(i \circ \Gamma)\left(a_{1}\right),(i \circ \Gamma)\left(b_{1}\right),(i \circ \Gamma)\left(a_{2}\right)$ and $(i \circ \Gamma)\left(b_{2}\right)$ respectively.

Observation 2.2.3. Note that for $a_{i}, b_{i}$ the relations given in Corollary 2.1.3.1 and Lemma 2.1.4 hold.

The next lemma shows an identities that is useful for further computations.

Lemma 2.2.4. For $s \in \mathbb{Z}$ it holds

$$
\text { 1. } \sigma b_{1}^{s} \sigma^{-1}=\left(b_{2}^{-1} a_{2} b_{2} a_{2}\right)^{\frac{1}{2}\left(1-(-1)^{s}\right)} b_{1}^{s} \text {. }
$$

Proof. Note that $\sigma b_{1}^{s} \sigma^{-1}=\left(b_{2}^{-1} a_{2} b_{2} a_{2} b_{1}\right)^{s}$. The following holds, where we use relations 4 and 5 of Theorem 2.2.2

$$
\left(b_{2}^{-1} a_{2} b_{2} a_{2} b_{1}\right)^{2}=\left(b_{2}^{-1} a_{2} b_{2} a_{2} b_{1}\right)\left(b_{2}^{-1} a_{2} b_{2} a_{2} b_{1}\right)=\left(b_{2}^{-1} a_{2} b_{2} a_{2}\right)\left(a_{2} b_{2} a_{2}\right)^{-1} a_{2}^{-1}\left(a_{2} b_{2} a_{2}\right) a_{2}^{-1} b_{1}^{2}=b_{1}^{2} .
$$

Since $b_{1}^{2}$ commutes with $a_{2}$ and $b_{2}$ it follows for $s \in \mathbb{Z}$

$$
\begin{aligned}
\left(b_{2}^{-1} a_{2} b_{2} a_{2} b_{1}\right)^{2 s} & =\left[\left(b_{2}^{-1} a_{2} b_{2} a_{2} b_{1}\right)^{2}\right]^{s}=b_{1}^{2 s} \\
\left(b_{2}^{-1} a_{2} b_{2} a_{2} b_{1}\right)^{2 s+1} & =\left(b_{2}^{-1} a_{2} b_{2} a_{2} b_{1}\right)\left(b_{2}^{-1} a_{2} b_{2} a_{2} b_{1}\right)^{2 s}=\left(b_{2}^{-1} a_{2} b_{2} a_{2}\right) b_{1}^{2 s+1} .
\end{aligned}
$$

From Theorem 2.2.2 it follows that every element $\alpha$ in $B_{2}(K)$ can be written in the form $\alpha=$ $w a_{1}^{r} b_{1}^{s} \sigma^{\epsilon}$, where $w$ is a word in the free group $F_{2}$ on two generators, $a_{1}^{r} b_{1}^{s} \in K=\left\langle a_{1}, b_{1}: b_{1}^{-1} a_{1} b_{1} a_{1}\right\rangle$ and $\epsilon$ is either zero or one. We call this form the normal form in $B_{2}(K)$ and it holds that two elements are equal if their normal form is equal. Note that $\epsilon$ is zero if and only if $\alpha \in F_{2} \rtimes_{\theta_{1}} \pi_{1}(K)$, that is, a pure braid. To compute the normal form we use the relations of the presentation, to spare us some computations later we compute in the next lemma the normal form of a multiplication between two certain braids.

Lemma 2.2.5. Let $\alpha=w_{1} a_{1}^{r_{1}} b_{1}^{s_{1}} \sigma$ and $\beta=w_{2} a_{1}^{r_{2}} b_{1}^{s_{2}} \sigma$ be two elements in $B_{2}(K)$ with $\operatorname{pr}\left(w_{i}\right)=$ $a^{m_{i}} b^{n_{i}}$, where pr: $F_{2} \rightarrow F_{2} /\left\langle a_{2} b_{2} a_{2} b_{2}^{-1}\right\rangle$ is the projection. Then

$$
\alpha \beta=w a_{1}^{r_{1}+(-1)^{s_{1}} m_{2}+(-1)^{s_{1}+n_{2}} r_{2}} b_{1}^{s_{1}+n_{2}+s_{2}},
$$

where $w \in F_{2}$ such that $\operatorname{pr}(w)=a_{2}^{m_{1}-(-1)^{s_{1}+m_{1}} m_{2}}$.

Proof. Recall that by Observation 2.1.5 the map $p r$ and the homomorphism $\theta\left(a_{1}^{r} b_{1}^{s}\right)$ commute for every $r, s \in \mathbb{Z}$. Hence $p r \times i d: F_{2} \rtimes_{\theta_{1}} \pi_{1}(K) \rightarrow F_{2} /\left\langle a_{2} b_{2} a_{2} b_{2}^{-1}\right\rangle \rtimes_{\theta_{1}} \pi_{1}(K)$ is a homomorphism. We 
compute the normal form of $(p r \times i d)(\alpha \beta)$. Note first that

$$
\begin{aligned}
(p r \times i d)\left(\sigma w_{2}\left(a_{2}, b_{2}\right) \sigma^{-1}\right) & =(p r \times i d)\left(w_{2}\left(\sigma a_{2} \sigma^{-1}, \sigma b_{2} \sigma^{-1}\right)\right) \\
& =(p r \times i d)\left(w_{2}\left(b_{2}^{-1} a_{2} b_{2} a_{1}, b_{2}^{-1} a_{2} b_{2} a_{2} b_{1}\right)\right) \\
& =(p r \times i d)\left(w_{2}\left(a_{2}^{-1} a_{1}, b_{1}\right)\right) \\
& =a_{2}^{-m_{2}} a_{1}^{m_{2}} b_{1}^{n_{2}} .
\end{aligned}
$$

The last step follows from the fact that $b_{1}$ acts on $a_{2}$ as on $a_{1}$, i.e. $b_{1} a_{i}=a_{i}^{-1} b_{1}$ for $i \in\{1,2\}$. Therefore

$$
\begin{aligned}
(p r \times i d)(\alpha \beta) & =(p r \times i d)\left(w_{1} a_{1}^{r_{1}} b_{1}^{s_{1}} w_{2} a_{1}^{r_{2}} b_{1}^{s_{2}}\right) \\
& =(p r \times i d)\left(w_{1} a_{1}^{r_{1}} b_{1}^{s_{1}}\right)(p r \times i d)\left(\sigma w_{2} \sigma^{-1}\right)(p r \times i d)\left(\left(a_{1}\right)^{r_{2}}\left(b_{2}^{-1} a_{2} b_{2} a_{2} b_{1}\right)^{s_{2}}\left(b_{2}^{-1} a_{2} b_{2} a_{2}\right)\right) \\
& =\left(a_{2}^{m_{1}} b_{2}^{m_{1}}\right)\left(a_{1}^{r_{1}} b_{1}^{s_{1}}\right)\left(a_{2}^{-m_{2}} a_{1}^{m_{2}} b_{1}^{n_{2}}\right)\left(\left(a_{1}\right)^{r_{2}} b_{1}^{s_{2}}\right) \\
& =\left(a_{2}^{m_{1}-(-1)^{s_{1}+m_{1}} m_{2}} b_{2}^{m_{1}}\right)\left(a_{1}^{r_{1}+(-1)^{s_{1}} m_{2}+(-1)^{s_{1}+n_{2}} r_{2}} b_{1}^{s_{1}+n_{2}+s_{2}}\right) .
\end{aligned}
$$

Therefore

$$
\alpha \beta=w\left(a_{1}^{r_{1}+(-1)^{s_{1}} m_{2}+(-1)^{s_{1}+n_{2}} r_{2}} b_{1}^{s_{1}+n_{2}+s_{2}}\right),
$$

where $\operatorname{pr}(w)=\left(a_{2}^{m_{1}-(-1)^{s_{1}+m_{1}} m_{2}} b_{2}^{m_{1}}\right)$. 


\section{Chapter 3}

\section{Nielsen fixed point theory on $n$-valued maps}

In this chapter we give a brief introduction to Nielsen fixed point theory for multivalued maps. Furthermore we a observe that a formula for computing the Nielsen number for 2-valued maps developed in [GG17] for orientable manifolds also holds for nonorientable manifolds (see Proposition 3.2.7). Also we make a connection between the 2-valued maps and homotopy classes of maps which do not satisfies the Borsuk-Ulam property, as discussed in Proposition 3.2.5.

\subsection{Multivalued maps and Configuration space}

A multifunction between two topological spaces $X$ and $Y$ is, according to chapter II of [Ber97], a correspondence $\phi$ that associates to every $x \in X$ a subset of $Y$. For a multifunction $\phi$ between $X$ and $Y$ we use the notation $\phi: X \multimap Y$. A multifunction $\phi: X \multimap Y$ is upper-semi-continuous if $\phi(x)$ for all $x \in X$ and if for each open set $V \subset Y$ with $\phi(x) \subset V$ there exists an open neighbourhood of $x$ such that $\phi(U) \subset V$ and lower-semi-continuous if for every $x \in X$ and every open set $V \subset Y$ with $\phi(x) \subset V$ there exists an open neighbourhood $U$ of $x$ such that for every $z \in U$ it holds that $\phi(z) \cap U \neq \emptyset$. A multifunction is continuous if it is both upper-semi-continuous and lowersemi-continuous. We refer to a continuous multifunction as multimap. A multifunction $\phi$ is called $n$-valued if for each $x \in X$ the subset $\phi(x)$ in $Y$ consists of exactly $n$ points. From now on we only consider (continuous) $n$-valued maps.

A homotopy between two $n$-valued maps $\hat{\Phi}: X \multimap Y$ and $\phi_{2}: X \multimap Y$ is an $n$-valued map $H: X \times I \multimap Y$ such that $H(x, 0)=\hat{\Phi}(x)$ and $H(x, 1)=\phi_{2}(x)$ for every $x \in X$.

We say that an $n$-valued function $\phi: X \multimap Y$ splits (into disctinct maps) if there are $n$ (simplevalued) continuous maps $f_{1}, \ldots f_{n}: X \rightarrow Y$ such that $\phi(x)=\left\{f_{1}(x), \ldots, f_{n}(x)\right\}$ for every $x \in X$. If this is the case we write $\phi=\left\{f_{1}, \ldots, f_{n}\right\}$. Note that if an $n$-valued map is split, then it is continuous.

One crucial tool to define Nielsen theory for $n$-valued maps is the Splitting Lemma, proofed by H. Schirmer.

Lemma 3.1.1 (Splitting Lemma). [Sch84a, Lemma 1] Let $X$ and $Y$ be compact Hausdorff. If $X$ is path connected and simply connected and $\Phi: X \multimap Y$ a (continuous) n-valued map, then $\Phi$ splits into $n$ distinct maps.

Denote the (ordered) configuration space of $Y$ defined by

$$
F_{n}(Y)=Y^{n} \backslash\left\{\left(y_{1}, \ldots, y_{n}\right): y_{i} \neq y_{j}, i \neq j\right\} .
$$

Consider the free action of the symmetric group $\mathcal{S}_{n}$ given by

$$
\begin{aligned}
\mathcal{S}_{n} \times F_{n}(Y) & \rightarrow F_{n}(Y) \\
\left(\sigma,\left(y_{1}, \ldots, y_{n}\right)\right) & \mapsto\left(y_{\sigma(1)}, \ldots, y_{\sigma(n)}\right) .
\end{aligned}
$$


We define the unordered configuration space of $Y$ as the quotient $D_{n}(Y)=F_{n}(Y) / \mathcal{S}_{n}$ and denote the quotient map by $\pi: F_{n}(Y) \rightarrow D_{n}(Y)$. Note that there is a natural bijection between the $n$ point subsets of a space $Y$ and the unordered configuration space $D_{n}(Y)$. This induces a bijective correspondence between $n$-valued functions from $X$ to $Y$ and functions from $X$ to $D_{n}(Y)$, see for example [GG18] From now on we write $\Phi$ for the function from $X$ to $Y$ that corresponds to the $n$-valued function $\phi: X \multimap Y$. R. Brown and D. L. Gonçalves show in [BG18, Corollary 4.1] that if $X$ and $Y$ are metric spaces, an $n$-valued function $\phi: X \multimap Y$ is continuous if and only if the correspondent map $\Phi: X \rightarrow D_{n}(Y)$ is continuous.

We say that a map $\hat{\Phi}: X \rightarrow F_{n}(Y)$ is a lift of a $n$-valued map, if the following diagram commutes.

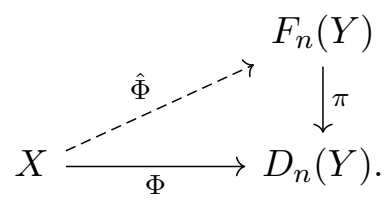

Lemma 3.1.2. [GG18, page 8] Let $X$ and $Y$ be metric spaces. An n-valued map $\phi$ admits a lift if and only if $\phi$ is split.

Observation 3.1.3. Since $\left(F_{n}(Y), \pi\right)$ is a covering space of $D_{n}(Y)$ the map $\phi$ admits a lift if and only if $\Phi$ admits a lifting, but this is equivalent to $\Phi_{\#}\left(\pi_{1}(X)\right) \subset \pi\left(\pi_{1}\left(F_{n}(Y)\right)\right)$ (see [Mas67, Chapter $V$ Theorem 5.1]). Recall that $\pi_{1}\left(D_{n}(Y)\right)$ is the total braid group $B_{n}(Y)$ and $\pi_{1}\left(F_{n}(Y)\right)$ is the pure braid group $P_{n}(Y)$. Therefore $\phi: X \multimap Y$ admits a lift if and only if $\Phi_{\#}$ sends every element to a pure braid. Hence by the previous Lemma $\phi$ splits if and only if $\Phi_{\#}\left(\pi_{1}(X)\right) \subset P_{n}(Y)$, see [BG18, Theorem 3.1].

\subsection{Nielsen number of 2-valued maps}

H. Schirmer studies $n$-valued maps on compact polyhedra. She defines in [Sch84b] an index, Nielsen classes and number and shows in [Sch85] that every $n$-valued map $\phi: X \multimap X$ is homotopic to a map $\hat{\phi}$ that has $N(\phi)$ fixed points. A fixed point of an $n$-valued map $\phi: X \multimap X$ is a point $x \in X$ such that $x \in \phi(x)$, that is Fix $(\phi)=\{x \in X: x \in \phi(x)\}$. In regard to Nielsen fixed point theory $n$-valued maps behave a lot like single valued maps. To define an equivalence relation in Fix $(\phi)$ let $x_{1}$ and $x_{2}$ be fixed points of $\phi$ and let $\lambda: I \rightarrow X$ be a path between $x_{1}$ and $x_{2}$. Since $I$ satisfies the conditions of the Splitting Lemma 3.1.1 there are $n$ (single-valued) maps $g_{1}, \ldots, g_{n}: \lambda(I) \rightarrow X$ such that $\phi_{\mid \lambda(I)}=\left\{g_{1}, \ldots, g_{n}\right\}$. We say that $x_{1}$ and $x_{2}$ are equivalent if there is an $i \in\{1, \ldots, n\}$ such that $g_{i} \circ \lambda$ is a path from $x_{1}$ to $x_{2}$ that is homotopic to $\lambda$.

Using analogies of the Simplicial Approximation Theorem and the Hopf Theorem H. Schirmer shows that, if $X$ is a compact polyhedra, every $n$-valued map $\phi: X \multimap X$ is homotopic to a map $\hat{\phi}: K \multimap K$ such that set Fix $(\hat{\phi})$ is finite and every fixed point is isolated (see [Sch84a, Theorem $6])$.

We start defining an index for $\hat{\phi}$, for that suppose that $x \in F i x(\hat{\phi})$. Since $x$ is an isolated fixed point there is a contractible open neighbourhood $U \subset X$ of $x$ such that $U \cap \operatorname{Fix}(\phi)=\{x\}$. By the Splitting Lemma there are maps $f_{1}, \ldots, f_{n}: U \rightarrow X$ such that $\hat{\phi}_{\mid U}=\left\{f_{1}, \ldots, f_{n}\right\}$. Since $x \in \hat{\phi}(x)=\left\{f_{1}(x), \ldots, f_{n}(x)\right\}$ there is exactly one $i \in\{1, \ldots, n\}$ such that $f_{i}(x)=x$. Define the index of $\hat{\phi}$ as $\operatorname{ind}(\hat{\phi}, x)=\operatorname{ind}\left(f_{i}, x\right)$. Let $V$ be an open set with $F i x(\hat{\phi}) \cap V=\left\{x_{1}, \ldots, x_{m}\right\}$, then define the fixed point index of $\hat{\phi}$ on $V$ as ind $(\hat{\phi}, V)=\sum_{j=1}^{m} i n d\left(f_{i}, x_{j}\right)$. With this definition the ind is a homotopy invariant [Sch84b, Lemma 3.2] and fulfils the axioms necessary for being an index.

\subsubsection{Split maps}

For a split map H. Schirmer provides a formula for the Nielsen number as stated in the following Proposition. 
Proposition 3.2.1. [Sch84b, Corollary 7.2] Let $X$ be a compact polyhedron and let $\phi=\left\{f_{1}, \ldots, f_{n}\right\}$ : $X \multimap X$ be an $n$-valued split map. Then the Nielsen number of $\phi$ is given by

$$
N(\phi)=\sum_{i=1}^{n} N\left(f_{i}\right) .
$$

Let $K$ be the Klein-bottle with fundamental group $\pi_{1}(K)=\langle\alpha, \beta: \alpha \beta \alpha=\beta\rangle$, then we can separate a map on the Klein bottle in two types, as suggested in the following lemma. For a proof see for example [GK06, Lemma3.1.].

Lemma 3.2.2. There are two types of self-maps on the Klein-bottle

$$
\begin{array}{cc}
f \text { is of type }(I) \text { if } & f \text { is of type }(I I) \text { if } \\
f_{\#}: \alpha \longmapsto \alpha^{r} & f_{\#}: \alpha \longmapsto 1 \\
\beta \longmapsto \alpha^{s} \beta^{2 t+1}, & \beta \longmapsto \alpha^{s} \beta^{2 t},
\end{array}
$$

where $r, s, t \in \mathbb{Z}$.

Since the Klein-bottle is a $K(\pi, 1)$ space $f: K \rightarrow K$ is completely given by the homomorphism induced on the fundamental group. We write $f=(r, s, t)$ for a map $f: K \rightarrow K$ such that

$$
\begin{aligned}
f_{\#}: \pi_{1}(K) & \longrightarrow \pi_{1}(K) \\
\alpha & \longmapsto \alpha^{r} \\
\beta & \longmapsto \alpha^{s} \beta^{t} .
\end{aligned}
$$

The next proposition states provides a formula for the Nielsen number of a selfmap on the Klein bottle, see for example [KLY08, Lemma 2.3.] for a proof.

Proposition 3.2.3. Let $f: K \rightarrow K$ be given by $f=(r, s, t)$, then the Nielsen number of $f$ is

$$
N(f)= \begin{cases}|(t-1) r| & \text { if } t \text { is odd and } r \neq 0 \\ |t-1| & \text { otherwise. }\end{cases}
$$

\subsubsection{Non-split maps}

D. L. Gonçalves and J. Guaschi give a formula for the Nielsen number of $n$-valued maps on connected, closed orientable manifolds (see [GG17]). We follow this idea for 2-valued maps to generlize for connected, closed manifolds. Let $X$ be a connected closed manifold and let $\phi: X \multimap$ $X$ be a 2-valued map that is not split. Consider the correspondent map $\Phi: X \rightarrow D_{2}(X)$. By Observation $3.1 .3 \mathrm{im}\left(\Phi_{\#}\right) \not \subset P_{2}(X)$, thus $H=\Phi_{\#}^{-1}\left(P_{2}(X)\right)$ is a proper subset of $\pi_{1}(X)$. Also $X$ admits a universal covering, therefore there is a covering $q: \tilde{X} \rightarrow X$ correspondent to the subgroup $H$. Since $P_{2}(X)$ is a normal subgroup of $B_{2}(X)$ of index two $H$ is a normal subgroup of $\pi_{1}(X)$ of index two and $q: \tilde{X} \rightarrow X$ is a double covering. Indeed consider the homomorphism $\tau \circ \Phi_{\#}: \pi_{1}(X) \rightarrow \mathcal{S}_{2}$, where $\tau: B_{2}(X) \rightarrow \mathcal{S}_{2}$ is the map that associates a braid to a permutation as seen in 2.1. Since the kernel of $\tau$ is $P_{2}(X)$ it holds that $H=\Phi_{\#}^{-1}\left(P_{2}(X)\right)$ is the kernel of $\tau \circ \Phi_{\#}$. Also $\Phi_{\#}\left(\pi_{1}(K)\right)$ is not contained in $P_{2}(X)$ split, hence $\tau \circ \Phi_{\#}$ is surjective and induces an isomorphism $\Gamma: \pi_{1}(K) / H \rightarrow \mathcal{S}_{2}$. Let $\delta \in \pi_{1}(K) / H$ that is associated to $(12) \in \mathcal{S}_{2}$, then $\delta$ is the (unique) Deck transformation that takes a point $\tilde{x}_{1}$ of the fiber $q^{-1}(x)=\left\{\tilde{x}_{1}, \tilde{x}_{2}\right\}$ of $x \in X$ to $\tilde{x}_{2}$, the other point of the fiber.

Also it holds that $(\Phi \circ q)_{\#}(\tilde{X}) \subset P_{2}(X)$, therefore there is a lifting $\hat{\Phi}: X \rightarrow F_{2}(Y)$ of $\Phi \circ q$.

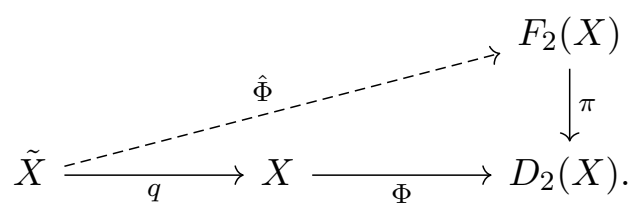


From Proposition 16 of [GG18] it follows that there $\Phi \circ q$ admits exactly two liftings of $\Phi \circ q$, if $\hat{\Phi}=\left(\hat{f}_{1}, \hat{f}_{2}\right)$ is a lifting, then $(12) \hat{\Phi}=\left(\hat{f}_{2}, \hat{f}_{1}\right)$ is the other one. Fix the base points $x_{0} \in K$ and $\left.\left\{p_{1}, p_{2}\right\} \in D_{(} K\right)$ with $\Phi\left(x_{0}\right)=\left\{p_{1}, p_{2}\right\}$ and $\tilde{x}_{0} \in q^{-1}\left(x_{0}\right)$. Then there is a unique lifting that takes $\tilde{x}_{0}$ to $\left(p_{1}, p_{2}\right)$. Without loss of generalities we consider, if not otherwise stated, $\hat{\Phi}$ to be this lifting.

Observation 3.2.4. Let $\phi, \Phi, q: \tilde{X} \rightarrow X$ and $\delta: \tilde{X} \rightarrow \tilde{X}$ be as above. Consider the covering $\pi: F_{2}(X) \rightarrow D_{2}(X)=F_{2}(X) / \mathcal{S}_{2}$ has exactly one non-trivial deck, which is (12). Using the fact that $q$ and $\pi$ are covering maps and $\hat{\Phi}=\left(\hat{f}_{1}, \hat{f}_{2}\right)$ is a lifting of $\Phi \circ q$ it follows that $\hat{\Phi}$ is equivariant, that is we have

$$
\hat{\Phi}(\delta \cdot \tilde{x})=\Gamma(\delta) \cdot \hat{\Phi}(\tilde{x}) .
$$

Thus

$$
\begin{aligned}
\left(\hat{f}_{1}(\delta \cdot \tilde{x}), \hat{f}_{2}(\delta \cdot \tilde{x})\right) & =\hat{\Phi}(\delta \cdot \tilde{x}) \\
& =(12) \cdot \hat{\Phi}(\tilde{x}) \\
& =(12) \cdot\left(\hat{f}_{1}(\tilde{x}), \hat{f}_{2}(\tilde{x})\right) \\
& =\left(\hat{f}_{2}(\tilde{x}), \hat{f}_{1}(\tilde{x})\right) .
\end{aligned}
$$

Therefore $\hat{f}_{1}(\delta \cdot \tilde{x})=\hat{f}_{2}(\tilde{x})$ for every $\tilde{x} \in \tilde{X}$.

Let $\tau_{0}: X \rightarrow X$ be a free involution. We say that a free homotopy class $[f] \in[X, Y]$ has the Borsuk-Ulam Property with respect to $\tau_{0}$ if and only if for every $g \in[f]$ there exists an $x \in X$ such that $g(x)=\left(g \circ \tau_{0}\right)(x)$. Hence, for a homotopy class $[f] \in[X, Y]$ the Borsuk-Ulam Property does not hold in respect to $\tau_{0}$ if and only if there is a map $f_{1} \in[f]$ such that $\operatorname{Coin}\left(f_{1}, f_{1} \circ \tau_{0}\right)=\emptyset$.

Proposition 3.2.5. Let $\Phi: X \rightarrow D_{2}(Y)$ be a map, $q: \tilde{X} \rightarrow X$ be the covering space induced by the subgroup $\Phi_{\#}^{-1}\left(P_{2}(Y)\right) \subsetneq \pi_{1}(X)$ with Deck transformation $\delta: \tilde{X} \rightarrow \tilde{X}$ and let $\hat{\Phi}=\left(\hat{f}_{1}, \hat{f}_{2}\right): \tilde{X} \rightarrow$ $F_{2}(Y)$ be a lifting of $\Phi \circ q$. Then

$$
\hat{f}_{2}=\hat{f}_{1} \circ \delta
$$

and $\left[\hat{f}_{1}\right]$ does not have the Borsuk-Ulam Property in respect to $\delta$.

Conversely if $[\hat{f}] \in[\tilde{X}, Y]$ is a homotopy class that does not have the Borsuk-Ulam Property, then there is a map $\hat{f}_{1} \in[\hat{f}]$ and a map $\Phi: X \rightarrow D_{2}(Y)$ such that $\left(\hat{f}_{1}, \hat{f}_{1} \circ \delta\right): \tilde{X} \rightarrow F_{2}(Y)$ is a lifting of $\Phi \circ q$.

Proof. For the first part it follows from Observation 3.2.4 that $\hat{f}_{2}=\hat{f}_{1} \circ \delta$. Also, since $\left(\hat{f}_{1}, \hat{f}_{2}\right)$ : $\tilde{X} \rightarrow F_{2}(Y)$ is a map it follows that $\hat{f}_{1}$ and $\hat{f}_{2}$ do not coincide. Hence $\hat{f}_{1}(\tilde{x}) \neq\left(\hat{f}_{1} \circ q\right)(\tilde{x})$ for every $\tilde{x} \in \tilde{X}$ and the class $\left[\hat{f}_{1}\right]$ does not have the Borsuk-Ulam Propery in respect to $\delta$.

For the converse suppose that the class $[\hat{f}] \in[X, Y]$ does not have the Borsuk-Ulam Property in respect to $\delta$. Then there is a map $\hat{f}_{1} \in[\hat{f}]$, such that $\hat{f}_{1}(\tilde{x}) \neq\left(\hat{f}_{1} \circ \delta\right)(\tilde{x})$ for every $\tilde{x} \in \tilde{X}$, this is $\operatorname{Coin}\left(\hat{f}_{1}, \hat{f}_{1} \circ \delta\right)=\emptyset$. Set $\hat{\Phi}=\left(\hat{f}_{1}, \hat{f}_{1} \circ \delta\right)$, then $\hat{\Phi}$ is an equivariant map from $\tilde{X}$ to $F_{2}(Y)$. Indeed for every $\tilde{x} \in \tilde{X}$

$$
\begin{aligned}
\hat{\Phi}(\delta(\tilde{x})) & =\left(\hat{f}_{1}(\delta(\tilde{x})),\left(\hat{f}_{1} \circ \delta\right)(\delta(\tilde{x}))\right) \\
& \left.=\left(\hat{(} f_{1} \circ \delta\right)(\tilde{x}), \hat{f}_{1}(\tilde{x})\right) \\
& =(12) \hat{\Phi}(\tilde{x}) .
\end{aligned}
$$

Thus $\hat{\Phi}$ induces a map $\Phi: \tilde{X} \rightarrow D_{2}(Y)$ on the quotients and is a lifting of $\Phi \circ q$.

Since we are in non-orientable spaces a notion that is needed is of orientation-true maps. Let $X, Y$ be connected manifolds, we say a map $g: X \rightarrow Y$ is orientation-true, if given any loop $\gamma: I \rightarrow X$ the loop $g \circ \gamma: I \rightarrow Y$ preserves (reverses) orientation if and only if $\gamma$ preserves 
(reverses) orientation (see [BFGJ05, page 406]). As an example consider $X$ and $Y$ to be surfaces, then $f$ is orientation-true if it takes loops that go over a Möbius strip to loops that go over a Möbius strip and loops that don't to loops that don't.

Consider two maps $f, g: X \rightarrow Y$ with $g$ orientation-true, then the coincidence index for the pair $f, g$ of an isolated point is an integer defined as the local index of the pair $f, g$ [GJ97, Definition 5.1.]. entation-true guarantees that there is a consistent global choice of local orientations.

Lemma 3.2.6. A covering map $q \tilde{X} \rightarrow X$ is orientation-true.

Proof. Let $\tilde{x} \in X$ and let $\tilde{U} \subseteq \tilde{X}$ be an open neighbourhood small enough such that $\tilde{U}$ is homeomorphic to $U=q(\tilde{U})$ with a choosen orientation. Also let $\gamma: I \rightarrow \tilde{X}$ be a loop starting on $\tilde{x}$. The loop $\gamma$ transports $\tilde{U}$, as $q \circ \gamma$ transports $U$ and since $\tilde{U}$ and $U$ are homeomorphic, either the orientation changes for both, or for neither.

Corollary 23 of [GG17] implies that $N(\phi)=N\left(q, f_{1}\right)=N\left(q, f_{2}\right)$ for a non-split 2-valued map $\phi: X \multimap X$ with $X$ a connected closed orientable manifolds. The next Proposition is a generalization of this corollary to connected closed manifolds not necessarily orientable.

Proposition 3.2.7. Let $X$ be a closed connected manifold and let $\phi: X \multimap X$ be a non-split 2valued map with $q: \tilde{X} \rightarrow X$ the covering space defined by the subgroup $H$ as above and $\hat{\Phi}=\left(\hat{f}_{1}, \hat{f}_{2}\right)$ be the lifting of $\Phi \circ q$. Then

$$
N(\phi)=N\left(q, \hat{f}_{1}\right)=N\left(q, \hat{f}_{2}\right) .
$$

Proof. From Proposition 17 of [GG18] implies that $q$ restricts to a surjection

$$
q: \operatorname{Coin}\left(q, \hat{f}_{1}\right) \cup \operatorname{Coin}\left(q, \hat{f}_{2}\right) \rightarrow F i x(\phi) .
$$

Let $x \in \operatorname{Fix}(\phi)$ and $\tilde{x}_{1}, \tilde{x}_{2} \in q^{-1}(x)$. Then there exist $i, j \in\{1,2\}$ such that $\tilde{x}_{j} \in \operatorname{Coin}\left(q, \hat{f}_{i}\right)$. Let us say, without loss of generality, that $\tilde{x}_{1} \in \operatorname{Coin}\left(q, \hat{f}_{1}\right)$. We know from Observation 3.2.4 that $\hat{f}_{1}(\delta \cdot \tilde{x})=\hat{f}_{2}(\tilde{x})$ for every $\tilde{x} \in \tilde{X}$. Also $\delta \cdot \tilde{x}_{2}=\tilde{x}_{1}$, thus

$$
\hat{f}_{2}\left(\tilde{x}_{2}\right)=\hat{f}_{1}\left(\delta \cdot \tilde{x}_{2}\right)=\hat{f}_{1}\left(\tilde{x}_{1}\right)=q\left(\tilde{x}_{1}\right)=x,
$$

hence $\tilde{x}_{2} \in \operatorname{Coin}\left(q, \hat{f}_{2}\right)$. Therefore $\operatorname{Coin}\left(q, \hat{f}_{1}\right) \cap q^{-1}(x)$ and $\operatorname{Coin}\left(q, \hat{f}_{2}\right) \cap q^{-1}(x)$ are disjoint sets, both containing exactly one point and their union is $\left\{\tilde{x}_{1}, \tilde{x}_{2}\right\}$. Hence $q$ restricts to a bijection $q: \operatorname{Coin}\left(q, \hat{f}_{i}\right) \rightarrow \operatorname{Fix}(\phi)$ for both $i=1$ and $i=2$.

If two elements $\tilde{y}_{1}, \tilde{y}_{2}$ of $\operatorname{Coin}\left(q, \hat{f}_{i}\right)$, where $i \in\{1,2\}$, are in the same Nielsen coincidence, then by Lemma 18 of [GG17] $q\left(\tilde{y}_{1}\right)$ and $q\left(\tilde{y}_{2}\right)$ are in the same fixed point class of $\phi$. Furthermore, fixing an arbitrary $\tilde{x}_{0} \in \operatorname{Coin}\left(q, \hat{f}_{i}\right) \cap q^{-1}\left(x_{0}\right)$ restricts to a surjection $q:\left[\tilde{x}_{0}\right] \rightarrow\left[x_{0}\right]$ between the Nielsen coincidence class $\left[\tilde{x}_{0}\right]$ of $q$ and $\hat{f}_{i}$ and the Nielsen fixed point class $\left[x_{0}\right]$ of $\phi$. Since $q: \operatorname{Coin}\left(q, \hat{f}_{i}\right) \rightarrow \operatorname{Fix}(\phi)$ is bijective it follows that $q:\left[\tilde{x}_{0}\right] \rightarrow\left[x_{0}\right]$ is bijective also.

Let $i \in\{1,2\}, x \in F i x(\phi)$ be an isolated fixed point of $\phi$ and let $\tilde{x} \in q^{-1}(x) \cap \operatorname{Coin}\left(q, \hat{f}_{i}\right)$. Then there is a contractible neighbourhood $U \subset X$ of $x$ such that $F i x(\phi) \cap U=\{x\}$ and $q^{-1}(U)=\tilde{U} \cup \tilde{V}$, where $\tilde{U}$ and $\tilde{V}$ are disjoint open subsets of $\tilde{X}$ homeomorphic to $U$ by $q$. Suppose without loss of generality that $\tilde{x} \in \tilde{U}$. Now $U$ satisfies the conditions of the Splitting Lemma 3.1.1 and therefore there exist two maps $g_{1}, g_{2}: U \rightarrow X$ such that $\left.\phi\right|_{U}=\left\{g_{1}, g_{2}\right\}$. Since $\hat{\Phi}=\left(\hat{f}_{1}, \hat{f}_{2}\right): \tilde{X} \rightarrow F_{2}(X)$ is the lifting of $\Phi \circ q: \tilde{X} \rightarrow F_{2}(X)$ we have for every $y \in U$ that

$$
\left\{\hat{f}_{1}(y), \hat{f}_{2}(y)\right\}=\pi\left(\left(\hat{f}_{1}(y), \hat{f}_{2}(y)\right)\right)=\left\{\left(g_{1} \circ q\right)(y),\left(g_{2} \circ q\right)(y)\right\} .
$$

Suppose without loss of generality that $\left.g_{j} \circ q\right|_{\tilde{U}}=\left.\left(\hat{f}_{j}\right)\right|_{\tilde{U}}$, where $j \in\{1,2\}$. As $q$ restricts to a homeomorphism to $\tilde{U}$ we can write $g_{j}=\hat{f}_{j} \circ\left(\left.q\right|_{\tilde{U}}\right)^{-1}$. Since $\tilde{x} \in q^{-1}(x) \cap \operatorname{Coin}\left(q, f_{i}\right)$ it follows that $x \in \operatorname{Fix}\left(g_{i}\right)$. By definition of the fixed point index

$$
\operatorname{ind}(\phi, U)=\operatorname{ind}\left(g_{i}, U\right)=\operatorname{ind}\left(f_{i} \circ\left(\left.q\right|_{\tilde{U}}\right)^{-1}, U\right),
$$


which is exactly the local coincidence index of the pair $\left(q, \hat{f}_{i}\right)$ in $\tilde{U}$, where the local orientation in $\tilde{U}$ is determine by the local homeomorphism $q$. Now $q: \tilde{X} \rightarrow X$ is a covering map and by Lemma 3.2.6 is orientation-true. Thus the local index extends globally, that is the index of the class is the sum of the indices of all coincidence points contained in the class. Therefore the essential coincidence classes of the pair $q, \hat{f}_{i}$ correspond to the essential fixed point classes of $\phi$, implying that the Nielsen coincidence number $N\left(q, \hat{f}_{1}\right)$ and the Nielsen number $N(\phi)$ are equal.

It is possible to give a formula for the Nielsen number of an $n$-valued map $\phi$, where $n$ is greater than 2, in terms of Nielsen coincidence theory, generalizing Theorem 6 of [GG17].

\subsection{Coincidence number}

The 2-sheathed covering spaces of the Klein-bottle are the torus and the Klein bottle. So in order to compute the Nielsen fixed point number of a 2-valued non-split self-map on the Klein bottle it is necessary to compute the Nielsen coincidence number of maps from the Klein bottle to the Klein bottle and maps from the Torus to the Klein bottle. Also, every map $\left(f_{1}, f_{2}\right): X \rightarrow F_{2}(Y)$ satisfies that $f_{1}$ and $f_{2}$ do not coincide and consequently have Nielsen coincidence number zero.

To compute the Nielsen coincidence number of two maps on the Klein bottle $f_{1}$ and $f_{2}$ we use the formula D. L. Gonçalves and M. R. Kelly provide. Recall that by Lemma 3.2.2 a map $f: K \rightarrow K$ on the Klein bottle has two types and is given by $f=(r, p, q)$.

Proposition 3.3.1. [GK06] For two maps $f_{1}, f_{2}: K \rightarrow K$ given by

$$
\begin{aligned}
\left(f_{i}\right)_{\#}: \alpha & \rightarrow \alpha^{r_{i}} \\
\beta & \rightarrow \alpha^{s_{i}} \beta^{t_{i}} .
\end{aligned}
$$

Then the Nielsen coincidence number is given by

$$
N\left(f_{1}, f_{2}\right)=\left|t_{1}-t_{2}\right| \max \left\{\left|r_{1}\right|,\left|r_{2}\right|\right\} .
$$

Let $T$ be the Torus with fundamental group $\pi_{1}(T)=\langle a, b: a b=b a\rangle$.

Lemma 3.3.2. [GK08, Lemma 2.1] There are four types of homotopy classes $[f] \in[T, K]$ defined by the following induced homomorphisms from $\pi_{1}(T)$ to $\pi_{1}(K)$

$$
\begin{aligned}
& \text { Type I) } \\
& \text { Type IIa) } \\
& \text { Type IIb) } \\
& \text { Type IIc) }
\end{aligned}
$$

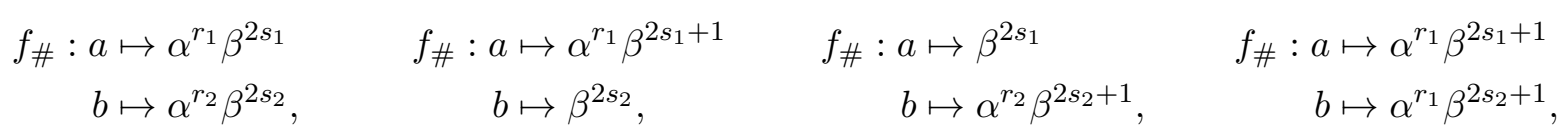

Proposition 3.3.3. [GK08, Lemma 2.1] Let $f, g: T \rightarrow K$ be two maps with induced homomorphism

$$
\begin{aligned}
f_{\#}: \pi_{1}(T) & \rightarrow \pi_{1}(K) \\
a & \mapsto \alpha^{r_{1}} \beta^{s_{1}} \\
b & \mapsto \alpha^{r_{2}} \beta^{s_{2}}, \\
g_{\#}: \pi_{1}(T) & \rightarrow \pi_{1}(K) \\
a & \mapsto \alpha^{t_{1}} \beta^{v_{1}} \\
b & \mapsto \alpha^{t_{2}} \beta^{v_{2}},
\end{aligned}
$$

then the Nielsen coincidence number $N(f, g)$ is given as follows

I) If both maps are of type I), then

$$
N(f, g)=\frac{1}{2}\left|\left(r_{1}-t_{1}\right)\left(s_{2}-v_{2}\right)-\left(r_{2}-t_{2}\right)\left(s_{1}-v_{1}\right)\right|+\frac{1}{2}\left|\left(r_{1}+t_{1}\right)\left(s_{2}-v_{2}\right)-\left(r_{2}+t_{2}\right)\left(s_{1}-v_{1}\right)\right| .
$$


II) If $g$ is of type I) and

(a) $f$ of type IIa) or IIb), then

$$
N(f, g)=\left|t_{1}\left(v_{2}-s_{2}\right)-t_{2}\left(v_{1}-s_{1}\right)\right|,
$$

c) if $f$ is of type IIc), then

$$
N(f, g)=2\left|t_{1}\left(v_{2}-s_{2}\right)-t_{2}\left(v_{1}-s_{1}\right)\right|,
$$

III) If $g$ is of type II), then $N(f, g)=0$. 


\section{Chapter 4}

\section{Homotopy classes of 2-valued split maps on the Klein bottle}

In this chapter we study 2-valued split maps, following the approach given by D. L. Gonçalves and J. Guaschi [GG18]. We first describe the homotopy classes of these maps in an algebraic way using the pure braid group and compute the Nielsen number. For maps with Nielsen number zero we provide a classification of the basedd homotpy classes.

As seen in chapter 3 there is a natural bijection between the set of 2-valued maps on $K$ and the set of continuous maps from $K$ on $D_{2}(K)$. This correspondence respects continuity and homotopy. Hence classifying the based homotopy classes of 2-valued maps on the Klein bottle $\left[\left(K, x_{0}\right),\left(K, x_{0}\right)\right]_{0}$ is the same as classifying $\left[\left(K, x_{0}\right),\left(D_{2}(K),\left(p_{1}, p_{2}\right)\right)\right]_{0}$, the set of homotopy classes of continuous maps between the based spaces $\left(K, x_{0}\right)$ and $\left(D_{2}(K),\left(p_{1}, p_{2}\right)\right)$. Since the Klein bottle $K$ is a CW complex and $D_{2}(K)$ is a $K(\pi, 1)$-space every homomorphism $\pi_{1}\left(K, x_{0}\right) \rightarrow \pi_{1}\left(D_{2}(K),\left\{p_{1}, p_{2}\right\}\right)$ is induced by a map $\left(K, x_{0}\right) \rightarrow\left(D_{2}(K),\left\{p_{1}, p_{2}\right\}\right)$ that is unique up to homotopy fixing $x_{0}$ (see for example A.Hatcher [Hat02, Page 90]). Note that the fundamental group of the unordered configuration space $\pi_{1}\left(D_{2}(K),\left\{p_{1}, p_{2}\right\}\right)$ is the total braid group $B_{2}(K)$. So there is a bijection between the set of based homotopy classes $\left[\left(K, x_{0}\right),\left(D_{2}(K),\left\{p_{1}, p_{2}\right\}\right)\right]_{0}$ and the set of homomorphisms $\operatorname{Hom}\left(\pi_{1}\left(K, x_{0}\right), B_{2}(K)\right)$, i.e.

$$
\left.\left[\left(K, x_{0}\right),\left(D_{2}(K),\left\{p_{1}, p_{2}\right\}\right)\right]_{0} \approx \operatorname{Hom}\left(\pi_{1}\left(K, x_{0}\right), B_{2}(K)\right)\right)
$$

Then the free homotopy classes are the quotient by conjugacy, where two homomorphisms $\varphi_{1}, \varphi_{2}$ are equivalent through conjugacy if there is a class of a loop $\delta \in \pi_{1}\left(D_{2}(K),\left\{p_{1}, p_{2}\right\}\right)=B_{2}(K)$ such that $\varphi_{1}=\delta * \varphi_{2} * \delta^{-1}$, where $\left(\delta * \varphi_{2} * \delta^{-1}\right)(\gamma)=\delta * \varphi_{2}(\gamma) * \delta^{-1}$.

In case that $\phi: K \multimap K$ is a split map, we can work on the pure braid group $P_{2}(K)$, instead of the total braid-group $B_{2}(K)$. In fact in chapter 3 we see that the map $\Phi: K \rightarrow D_{2}(K)$ with $\Phi\left(x_{0}\right)=\left\{p_{1}, p_{2}\right\}$ correspondent to $\phi$ admits a unique lift $\hat{\Phi}: K \rightarrow F_{2}(K)$ with $\hat{\Phi}\left(x_{0}\right)=\left(p_{1}, p_{2}\right)$. Therefore a first step to classify the homotopy classes of split maps on $K$ is to classify the homotpy classes of maps from $K$ to $F_{2}(K)$. The space $F_{2}(K)$ is also $K(\pi, 1)$ and it follows analogously that

$$
\begin{gathered}
{\left[\left(K, x_{0}\right),\left(D_{2}(K),\left\{p_{1}, p_{2}\right\}\right)\right]_{0} \approx \operatorname{Hom}\left(\pi_{1}\left(K, x_{0}\right),\left(B_{2}(K),\left\{p_{1}, p_{2}\right)\right\}\right) .} \\
{\left[K, F_{2}(K)\right] \approx \operatorname{Hom}\left(\pi_{1}(K), P_{2}(K)\right) / \sim,}
\end{gathered}
$$

Therefore we want to find the homomorphisms between $\pi_{1}\left(K, x_{0}\right)=\left\langle\alpha, \beta: \alpha \beta \alpha \beta^{-1}\right\rangle$ and $P_{2}(K)$. A homomorphism is determined by its image on the generators, in this case $\alpha$ and $\beta$. Also the relations have to be respected. In other words $\varphi: \pi_{1}(K) \rightarrow B_{2}(K)\left(P_{2}(K)\right.$, respectively) is a homomorphism, if and only if

$$
\varphi(\alpha) \varphi(\beta) \varphi(\alpha)=\varphi(\beta)
$$


Hence we want to find the pairs of braids $\hat{\alpha}, \hat{\beta}$ in $B_{2}(K)$ (or in $P_{2}(K)$ ), such that

$$
\hat{\alpha} \hat{\beta} \hat{\alpha}=\hat{\beta} .
$$

This is an algebraic problem, that can be stated by its own.

Assuming that $\hat{\alpha}$ and $\hat{\beta}$ are pure braids we give some partial solutions to this equation, restricting to the geometric cases that interests us the most. One restriction comes from supposing that $\phi$ has Nielsen number zero.

\subsection{Algebraic description and Nielsen number}

We start with some necessary conditions that equation (4.2) in $P_{2}(K)$ implies, to then study the classification of homotopy classes of maps from $K$ to $F_{2}(K)$ with Nielsen number zero. We identify $P_{2}(K)$ with $F_{2} \rtimes_{\theta_{1}} \pi_{1}(K)$ as in Corollary 2.1.3.1.

The first necessary conditions for solutions of equation 4.2 are obtained by considering the equation in each component of the semidirect product.

Proposition 4.1.1. Let $\hat{\alpha}=w_{1} a_{1}^{r_{1}} b_{1}^{s_{1}}$ and $\hat{\beta}=w_{2} a_{1}^{r_{2}} b_{1}^{s_{2}}$ be in $F_{2}\left(a_{2}, b_{2}\right) \rtimes_{\theta_{1}} \pi_{1}(K)$. Then

$$
\hat{\alpha} \hat{\beta} \hat{\alpha}=\hat{\beta}
$$

if and only if $s_{1}=0$ and one of the following cases holds

a) $s_{2}$ is even, $r_{1}=0$ and the following equation holds in $F_{2}$

$$
w_{2}=w_{1} w_{2} \theta_{1}\left(a_{1}^{r_{2}}\right)\left(w_{1}\right) .
$$

b) $s_{2}$ is odd and the following equation holds in $F_{2}$

$$
w_{2}=w_{1} \theta_{1}\left(a_{1}^{r_{1}}\right)\left(w_{2}\right) \theta_{1}\left(a_{1}^{r_{1}+r_{2}} b_{1}\right)\left(w_{1}\right) .
$$

Proof. Suppose that $\hat{\alpha} \hat{\beta} \hat{\alpha}=\hat{\beta}$, then

$$
\begin{aligned}
w_{2} a_{1}^{r_{2}} b_{1}^{s_{2}} & =\hat{\beta}=\hat{\alpha} \hat{\beta} \hat{\alpha}=w_{1} a_{1}^{r_{1}} b_{1}^{s_{1}} w_{2} a_{1}^{r_{2}} b_{1}^{s_{2}} w_{1} a_{1}^{r_{1}} b_{1}^{s_{1}} \\
& =w_{1} \theta_{1}\left(a_{1}^{r_{1}} b_{1}^{s_{1}}\right)\left(w_{2}\right) \theta_{1}\left(a_{1}^{r_{1}} b_{1}^{s_{1}} a_{1}^{r_{2}} b_{1}^{s_{2}}\right)\left(w_{1}\right) a_{1}^{r_{1}} b_{1}^{s_{1}} a_{1}^{r_{2}} b_{1}^{s_{2}} a_{1}^{r_{1}} b_{1}^{s_{1}} .
\end{aligned}
$$

In the semi-direct product $F_{2} \rtimes_{\theta_{1}} \pi_{1}(K)$ an element is equal to another if the components are equal. Thus in $F_{2}$ the following equation holds:

$$
w_{2}=w_{1} \theta_{1}\left(a_{1}^{r_{1}} b_{1}^{s_{1}}\right)\left(w_{2}\right) \theta_{1}\left(a_{1}^{r_{1}+(-1)^{s_{1}} r_{2}} b_{1}^{s_{1}+s_{2}}\right)\left(w_{1}\right) .
$$

In $\pi_{1}(K)$ we have

$$
a_{1}^{r_{2}} b_{1}^{s_{2}}=a_{1}^{r_{1}} b_{1}^{s_{1}} a_{1}^{r_{2}} b_{1}^{s_{2}} a_{1}^{r_{1}} b_{1}^{s_{1}}=a_{1}^{r_{1}+(-1)^{s_{1}} r_{2}+(-1)^{s_{1}+s_{2}} r_{1}} b_{1}^{2 s_{1}+s_{2}} .
$$

Written in the normal form in $\pi_{1}(K)$, two elements are equal in $\pi_{1}(K)$, if and only if the exponents of $a_{1}$ and $b_{1}$ are equal. Therefore from the exponents of $b_{2}$ it follows that $s_{2}=2 s_{1}+s_{2}$ thus $s_{1}=0$. From the exponents of $a_{2}$, it follows

$$
r_{2}=r_{1}+(-1)^{s_{1}} r_{2}+(-1)^{s_{1}+s_{2}} r_{1}=\left(1+(-1)^{s_{2}}\right) r_{1}+r_{2} .
$$

Therefore there are two cases, either $s_{2}$ is even and in this case $r_{1}=0$, or $s_{2}$ is odd.

If $s_{2}$ is even and consequently $r_{1}=0$ then, using Lemma 2.1.4, the equation (4.5) is equal to

$$
w_{2}=w_{1} w_{2} \theta_{1}\left(a_{1}^{r_{2}}\right)\left(w_{1}\right) .
$$


If $s_{2}$ is odd, then again from Lemma 2.1.4 it follows that equation (4.5) can be rewritten as

$$
w_{2}=w_{1} \theta_{1}\left(a_{1}^{r_{1}}\right)\left(w_{2}\right) \theta_{1}\left(a_{1}^{r_{1}+r_{2}} b_{1}\right)\left(w_{1}\right) .
$$

The converse follows from similar arguments.

To obtain more information about equations (4.3) and (4.4) we study this equation in a quotient of $F_{2}$. Consider the projection to the abelianization of the free group, which is the free abelian group isomorphic to $\mathbb{Z} \oplus \mathbb{Z}$.

$$
\begin{aligned}
A b: F_{2}\left(a_{2}, b_{2}\right) & \rightarrow \mathbb{Z} \oplus \mathbb{Z} \\
w & \rightarrow\left(|w|^{a_{2}},|w|^{b_{2}}\right),
\end{aligned}
$$

where $|w|^{a_{2}}$ and $|w|^{b_{2}}$ are the sums of the exponents of $a_{2}$ and $b_{2}$, respectively. That is, if

$$
w=\sum_{i=1}^{l} a_{2}^{x_{i}} b_{2}^{y_{i}},
$$

then

$$
|w|^{a_{2}}=\sum_{i=1}^{l} x_{i}
$$

and

$$
|w|^{b_{2}}=\sum_{i=1}^{l} y_{i} .
$$

Since $A b$ is a homomorphism the equations (4.3) and (4.4) have to hold still, after applying $A b$. This gives us more information about the equations.

Proposition 4.1.2. Let $w_{1}, w_{2} \in F_{2}$ satisfy equation (4.3), that is

$$
w_{2}=w_{1} w_{2} \theta_{1}\left(a_{1}^{r_{2}}\right)\left(w_{1}\right) .
$$

Then $\left|w_{1}\right|^{b_{2}}=0$ and $\left|w_{1}\right|^{a_{2}}=0$.

Proof. It follows from Lemma 2.1.4 that $\theta\left(a_{1}^{r_{2}}\right)\left(a_{2}\right)=a_{2}$ and $\theta\left(a_{1}^{r_{2}}\right)\left(b_{2}\right)=a_{2}^{2 r_{2}} b_{2}$. Since $\theta\left(a_{1}^{r_{2}}\right)$ is a homomorphism it holds that

$$
A b\left(\theta\left(a_{1}^{r_{2}}\right)\left(w_{1}\right)\right)=\left(\left|w_{1}\right|^{a_{2}}+2 r_{2}\left|w_{1}\right|^{b_{2}},\left|w_{1}\right|^{b_{2}}\right) .
$$

Hence applying $A b$ on equation (4.3) implies

$$
\begin{aligned}
\left(\left|w_{2}\right|^{a_{2}},\left|w_{2}\right|^{b_{2}}\right) & =A b\left(w_{2}\right) \\
& =A b\left(w_{1} w_{2} \theta_{1}\left(a_{1}^{r_{2}}\right)\left(w_{1}\right)\right) \\
& \left.=A b\left(w_{1}\right)+A b\left(w_{2}\right)+A b\left(\theta_{1}\left(a_{1}^{r_{2}}\right)\left(w_{1}\right)\right)\right) \\
& =\left(\left|w_{1}\right|^{a_{2}},\left|w_{1}\right|^{b_{2}}\right)+\left(\left|w_{2}\right|^{a_{2}},\left|w_{2}\right|^{b_{2}}\right)+\left(\left|w_{1}\right|^{a_{2}}+2 r_{2}\left|w_{1}\right|^{b_{2}},\left|w_{1}\right|^{b_{2}}\right) \\
& =\left(2\left|w_{1}\right|^{a_{2}}+\left|w_{2}\right|^{a_{2}}+2 r_{2}\left|w_{1}\right|^{b_{2}}, 2\left|w_{1}\right|^{b_{2}}+\left|w_{2}\right|^{b_{2}}\right) .
\end{aligned}
$$

Therefore $\left|w_{1}\right|^{b_{2}}=0$ and $\left|w_{1}\right|^{a_{2}}=0$.

Proposition 4.1.3. Let $w_{1}, w_{2} \in F_{2}$ satisfy equation (4.4), that is

$$
w_{2}=w_{1} \theta_{1}\left(a_{1}^{r_{1}}\right)\left(w_{2}\right) \theta_{1}\left(a_{1}^{r_{1}+r_{2}} b_{1}\right)\left(w_{1}\right),
$$


then $\left|w_{1}\right|^{b_{2}}=0$ and either $\left|w_{2}\right|^{b_{2}}=0$ or $r_{1}=0$.

Proof. As in the proof of Proposition 4.1.2 we have

$$
A b\left(\theta\left(a_{1}^{r_{1}}\right)\left(w_{2}\right)\right)=\left(\left|w_{2}\right|^{a_{2}}+2 r_{1}\left|w_{2}\right|^{b_{2}},\left|w_{2}\right|^{b_{2}}\right) .
$$

Using Lemma 2.1.4, it follows that $\theta\left(a_{1}^{r_{1}+r_{2}} b_{1}\right)\left(a_{2}\right)=a_{2}^{-1}$ and $\theta\left(a_{1}^{r_{1}+r_{2}} b_{1}\right)\left(b_{2}\right)=a_{2}^{2\left(r_{1}+r_{2}\right)+1} b_{2} a_{2}$. Therefore it holds

$$
A b\left(\theta\left(a_{1}^{r_{1}+r_{2}} b_{1}\right)\left(w_{1}\right)\right)=\left(-\left|w_{1}\right|^{a_{2}}+2\left(r_{1}+r_{2}+1\right)\left|w_{1}\right|^{b_{2}},\left|w_{1}\right|^{b_{2}}\right) .
$$

Applying the homomorphism $A b$ on equation (4.4) implies

$$
\begin{aligned}
\left(\left|w_{2}\right|^{a_{2}},\left|w_{2}\right|^{b_{2}}\right) & =A b\left(w_{2}\right) \\
& =A b\left(w_{1}\right) A b\left(\theta_{1}\left(a_{1}^{r_{1}}\right)\left(w_{2}\right)\right) A b\left(\theta_{1}\left(a_{1}^{r_{1}+r_{2}} b_{1}\right)\left(w_{1}\right)\right) \\
& =\left(\left|w_{1}\right|^{a_{2}},\left|w_{1}\right|^{b_{2}}\right)+\left(\left|w_{2}\right|^{a_{2}}+2 r_{1}\left|w_{2}\right|^{b_{2}},\left|w_{2}\right|^{b_{2}}\right)+\left(2\left(r_{1}+r_{2}+1\right)\left|w_{1}\right|^{b_{2}}-\left|w_{1}\right|^{a_{2}},\left|w_{1}\right|^{b_{2}}\right) \\
& =\left(\left|w_{2}\right|^{a_{2}}+2 r_{1}\left|w_{2}\right|^{b_{2}}+2\left(r_{1}+r_{2}+1\right)\left|w_{1}\right|^{b_{2}}, 2\left|w_{1}\right|^{b_{2}}+\left|w_{2}\right|^{b_{2}}\right) .
\end{aligned}
$$

Therefore $\left|w_{1}\right|^{b_{2}}=0$ and $r_{1}\left|w_{2}\right|^{b_{2}}=0$.

Solving the equations (4.3) and (4.4) is enough to solve equation (4.2) in $P_{2}(K)$ and therefore enough to classify the split maps from the Klein bottle $K$ to the ordered configuration space $F_{2}(K)$. Some solutions are straightforward, for example setting $w_{1}=1, r_{1}=0$ and taking an arbitrary $w_{2} \in F_{2}$ is a solution of equation (4.4). Finding all solutions of these equations ((4.3) and (4.4)) in the free group $F_{2}$ can be a lot of work, therefore we try to find further restrictions for these two equations, using the fact that they describe a map from $K$ to $F_{2}(K)$.

Let $\hat{\Phi}: K \rightarrow F_{2}(K)$ be a continuous map. Then there are maps $f_{1}, f_{2}: K \rightarrow K$ such that for every $x \in K$ it holds that $\hat{\Phi}(x)=\left(f_{1}(x), f_{2}(x)\right)$, that is $f_{1}=p r_{1} \circ \hat{\Phi}$ and $f_{2}=p r_{2} \circ \hat{\Phi}$, where $p r_{1}$ and $p r_{2}$ are the projections to the first, and to the second component, respectively. Since the image of $\hat{\Phi}$ is in $F_{2}(K)$ it holds that $f_{1}$ and $f_{2}$ cannot coincide at any point, i.e. $\operatorname{Coin}\left(f_{1}, f_{2}\right)=\emptyset$. Consider the induced map $\hat{\Phi}_{\#}: \pi_{1}(K) \rightarrow P_{2}(K)$. Suppose that the induced map $\hat{\Phi}_{\#}$ is given by

$$
\begin{aligned}
\hat{\Phi}_{\#}: \pi_{1}\left(K, p_{0}\right) & \longrightarrow F_{2} \rtimes_{\theta_{1}} \pi_{1}(K) \\
\alpha & \longmapsto \hat{\alpha} \\
\beta & \longmapsto \hat{\beta},
\end{aligned}
$$

where $\pi_{1}\left(K, p_{0}\right)=\langle\alpha, \beta: \alpha \beta \alpha=\beta\rangle$ with base point $p_{0}$. The elements $\hat{\alpha}$ and $\hat{\beta}$ are elements of $F_{2} \rtimes_{\theta_{1}} \pi_{1}(K)$ and therefore have the form

$$
\begin{aligned}
& \hat{\alpha}=w_{1} a_{1}^{r_{1}} b_{1}^{s_{1}} \\
& \hat{\beta}=w_{2} a_{1}^{r_{2}} b_{1}^{s_{2}},
\end{aligned}
$$

for some words $w_{1}, w_{2} \in F_{2}$ and integers $r_{1}, s_{1}, r_{2}, s_{2} \in \mathbb{Z}$. Since $\hat{\Phi}_{\#}$ is a homomorphism the equation (4.2) holds, i.e.

$$
\hat{\alpha} \hat{\beta} \hat{\alpha}=\hat{\beta} .
$$

Therefore Proposition 4.1.1 implies $s_{1}=0$ and either $s_{2}$ is even, $r_{1}=0$ and it holds

$$
w_{2}=w_{1} w_{2} \theta_{1}\left(a_{1}^{r_{2}}\right)\left(w_{1}\right),
$$


or $s_{2}$ is odd and it holds

$$
w_{2}=w_{1} \theta_{1}\left(a_{1}^{r_{1}}\right)\left(w_{2}\right) \theta_{1}\left(a_{1}^{r_{1}+r_{2}} b_{1}\right)\left(w_{1}\right) .
$$

Actually (4.1) guarantees that these conditions are not only necessary for $\hat{\Phi}$ to be a map from $K$ to $F_{2}(K)$, but also sufficient. Thus they ensure that $f_{1}$ and $f_{2}$ are coincidence-free. Nevertheless it is useful to take in account that $f_{1}$ and $f_{2}$ are coincidence-free, as this can give us information that could help us solve equations (4.3) and (4.4). Since $\operatorname{Coin}\left(f_{1}, f_{2}\right)=\emptyset$, the Nielsen coincidence number $N\left(f_{1}, f_{2}\right)$ is zero, hence we compute $N\left(f_{1}, f_{2}\right)$ in terms of $w_{1}, w_{2}, r_{1}, r_{2}$ and $s_{2}$ and set it zero. Before we can do that we determine $\left(f_{1}\right)_{\#}$ and $\left(f_{2}\right)_{\#}$. For that, note that $\hat{\Phi}_{\#}: \pi_{1}(K) \rightarrow P_{2}(K)$, using the isomorphism between $P_{2}$ and $F_{2} \rtimes_{\theta_{1}} \pi_{1}(K)$ given by Corollary 2.1.3.1, is actually

$$
\begin{aligned}
\hat{\Phi}_{\#}: \pi_{1}(K) & \longrightarrow P_{2}(K) \\
\alpha & \longmapsto w_{1}\left(a_{2} a_{1}\right)^{r_{1}} \\
\beta & \longmapsto w_{2}\left(a_{2} a_{1}\right)^{r_{2}}\left(b_{2} b_{1}\right)^{s_{2}} .
\end{aligned}
$$

Here $a_{1}$ and $b_{1}$ start and end at point $p_{1}$, where $a_{2}$ and $b_{2}$ start and end at $p_{2}$. The projections $p r_{1}$ and $p r_{2}$ "forget" the second and the first component, respectively. Therefore

$$
\begin{aligned}
\left(f_{1}\right)_{\#}=\left(p r_{1} \circ \hat{\Phi}\right)_{\#}: \pi_{1}\left(K, p_{0}\right) & \longrightarrow \pi_{1}\left(K, p_{1}\right) \\
\alpha & \longmapsto a_{1}^{r_{1}} \\
\beta & \longmapsto a_{1}^{r_{2}} b_{1}^{s_{2}}, \\
\left(f_{2}\right)_{\#}=\left(p r_{2} \circ \hat{\Phi}\right)_{\#}: \pi_{1}\left(K, p_{0}\right) & \longrightarrow \pi_{1}\left(K, p_{2}\right) \\
\alpha & \longmapsto \pi\left(w_{1}\right) a_{2}^{r_{1}} \\
\beta & \longmapsto \pi\left(w_{2}\right) a_{2}^{r_{2}} b_{2}^{s_{2}},
\end{aligned}
$$

where $\pi: F_{2} \rightarrow F_{2} \backslash\left\langle a_{2} b_{2} a_{2} b_{2}^{-1}\right\rangle=\pi_{1}(K)$ is the projection from the free group on two elements to the fundamental group of the Klein bottle.

Since $p_{0}, p_{1}$ and $p_{2}$ are pairwise different, $\pi_{1}\left(K, p_{0}\right)=\left\langle\alpha, \beta: \alpha \beta \alpha \beta^{-1}\right\rangle$ is isomorphic, but not equal to $\pi_{1}\left(K, p_{1}\right)=\left\langle a_{1}, b_{1}: a_{1} b_{1} a_{1} b_{1}^{-1}\right\rangle$ or to $\pi_{1}\left(K, p_{2}\right)=\left\langle a_{2}, b_{2}: a_{2} b_{2} a_{2} b_{2}^{-1}\right\rangle$. But it is possible to choose $p_{0}, p_{1}$ and $p_{2}$ and two isomorphisms, one that sends $\alpha$ and $\beta$ to $a_{1}$ and $b_{1}$, respectively and another one that sends $\alpha$ and $\beta$ to $a_{2}$ and $b_{2}$, respectively. Therefore it does not matters that $\pi_{1}\left(K, p_{0}\right), \pi_{1}\left(K, p_{1}\right)$ and $\pi_{1}\left(K, p_{2}\right)$ are pairwise distinct and we will write only $\pi_{1}(K)$ for all three.

To be able to write $\left(f_{2}\right)_{\#}(\alpha)$ and $\left(f_{2}\right)_{\#}(\beta)$ in their normal forms in $\pi_{1}\left(K, p_{2}\right)$ set $n_{1}, n_{2}, m_{1}, m_{2} \in$ $\mathbb{Z}$ such that

$$
\begin{aligned}
& \pi\left(w_{1}\right)=a_{2}^{m_{1}} b_{2}^{n_{1}}, \\
& \pi\left(w_{2}\right)=a_{2}^{m_{2}} b_{2}^{n_{2}} .
\end{aligned}
$$

Note that $n_{1}=\left|w_{1}\right|^{b_{2}}$ and $n_{2}=\left|w_{2}\right|^{b_{2}}$. Also, either equation (4.3) or equation (4.4) holds, therefore from the Propositions 4.1.2 and 4.1.3 it follows that $n_{1}=\left|w_{1}\right|^{b_{2}}=0$. Hence

$$
\begin{aligned}
\left(f_{2}\right)_{\#}: \pi_{1}(K) & \longrightarrow \pi_{1}(K) \\
\alpha & \longmapsto a_{2}^{m_{1}+r_{1}} \\
\beta & \longmapsto a_{2}^{m_{2}+(-1)^{n_{2}} r_{2}} b_{2}^{n_{2}+s_{2}} .
\end{aligned}
$$

Both $\left(f_{1}\right)_{\#}$ and $\left(f_{2}\right)_{\#}$ are homomorphisms on $\pi_{1}(K)$ and $f_{1}$ and $f_{2}$ are either of type $(I)$ or type $(I I)$, see Lemma 3.2.2. If $f_{1}$ and $f_{2}$ are of type $(I)$ it follows that $\left(f_{1}\right)_{\#}(\alpha)=1$ and $\left(f_{2}\right)_{\#}(\alpha)=1$ respectively.

Lemma 4.1.4. Let $\hat{\Phi}: K \rightarrow F_{2}(K)$ be a map and let $w_{1}, w_{2} \in F_{2}, r_{1}, r_{2}, s_{2} \in \mathbb{Z}$ be such that the 
induced map is

$$
\begin{aligned}
\hat{\Phi}_{\#}: \pi_{1}\left(K, p_{0}\right) & \longrightarrow F_{2} \rtimes_{\theta_{1}} \pi_{1}(K) \\
\alpha & \longmapsto \hat{\alpha}=w_{1} a_{1}^{r_{1}} \\
\beta & \longmapsto \hat{\beta}=w_{2} a_{1}^{r_{2}} b_{1}^{s_{2}} .
\end{aligned}
$$

If $s_{2}$ is even, then $r_{1}=0$, and if $s_{2}$ and $n_{2}$ are either both even or both odd, then $m_{1}=-r_{1}$.

Proof. Consider the maps $f_{1}=p r_{1} \circ \hat{\Phi}$ and $f_{2}=p r_{2} \circ \hat{\Phi}$. Then $\left(f_{1}\right)_{\#}$ and $\left(f_{2}\right)_{\#}$ are either of type $(I)$ or of type $(I I)$. From Proposition 4.1 .1 we already know that if $s_{2}$ is even, then $r_{1}=0$, but it follows also from the fact that in case that $s_{2}$ is even, $f_{1}$ is of type $(I)$, therefore $\left(f_{1}\right)_{\#}(\alpha)=1$. If $s_{2}$ and $n_{2}$ are both even or both odd, then $f_{2}$ is of type $(I)$, hence $\left(f_{2}\right)_{\#}(\alpha)=1$ and it follows that $m_{1}=-r_{1}$.

Computing the Nielsen coincidence number and setting it zero guarantees the following necessary conditions for maps from $K$ to $F_{2}(K)$.

Proposition 4.1.5. Let $\hat{\Phi}: K \rightarrow F_{2}(K)$ be a map and let $w_{1}, w_{2} \in F_{2}, r_{1}, r_{2}, s_{2} \in \mathbb{Z}$ be such that the induced map is

$$
\begin{aligned}
\hat{\Phi}_{\#}: \pi_{1}\left(K, p_{0}\right) & \longrightarrow F_{2} \rtimes_{\theta_{1}} \pi_{1}(K) \\
\alpha & \longmapsto \hat{\alpha}=w_{1} a_{1}^{r_{1}} \\
\beta & \longmapsto \hat{\beta}=w_{2} a_{1}^{r_{2}} b_{1}^{s_{2}},
\end{aligned}
$$

Then either $n_{2}$ is zero, or both $r_{1}$ and $m_{1}$ are zero.

Proof. Consider the maps $f_{1}=p r_{1} \circ \hat{\Phi}$ and $f_{2}=p r_{2} \circ \hat{\Phi}$. Then

$$
\begin{aligned}
\left(f_{1}\right)_{\#}: \pi_{1}(K) & \longrightarrow \pi_{1}(K) \\
\alpha & \longmapsto a_{2}^{r_{1}} \\
\beta & \longmapsto a_{2}^{r_{2}} b_{2}^{s_{2}}, \\
\left(f_{2}\right)_{\#}: \pi_{1}(K) & \longrightarrow \pi_{1}(K) \\
\alpha & \longmapsto a_{2}^{m_{1}+r_{1}} \\
\beta & \longmapsto a_{2}^{m_{2}+(-1)^{n_{2}} r_{2}} b_{2}^{n_{2}+s_{2}} .
\end{aligned}
$$

The maps $f_{1}$ and $f_{2}$ are coincidance-free, therefore usingthe formula of the Nielsen number given in Proposition3.3.1,

$$
\begin{aligned}
0=N\left(f_{1}, f_{2}\right) & =\left|s_{2}-\left(n_{2}+s_{2}\right)\right| \max \left\{\left|r_{1}\right|,\left|m_{1}+r_{1}\right|\right\} \\
& =\left|n_{2}\right| \max \left\{\left|r_{1}\right|,\left|m_{1}+r_{1}\right|\right\} .
\end{aligned}
$$

Hence either $n_{2}$ is zero, or both $r_{1}$ and $m_{1}$ are zero.

In summary, Propositions 4.1.1, 4.1.2, 4.1.3, 4.1.5 and Lemma 4.1.4 provide the following Theorem.

Theorem 4.1.6. Let $\hat{\Phi}: K \rightarrow F_{2}(K)$, then there are words $w_{1}$ and $w_{2}$ in the free group $F_{2}$ and integers $r_{1}, r_{2}, s_{2} \in \mathbb{Z}$ such that

$$
\begin{aligned}
\hat{\Phi}_{\#}: \pi_{1}\left(K, p_{0}\right) & \longrightarrow F_{2} \rtimes_{\theta_{1}} \pi_{1}(K) \\
\alpha & \longmapsto \hat{\alpha}=w_{1} a_{1}^{r_{1}} \\
\beta & \longmapsto \hat{\beta}=w_{2} a_{1}^{r_{2}} b_{1}^{s_{2}} .
\end{aligned}
$$

Also one of the following cases holds 
a) $s_{2}$ is even, $n_{1}=\left|w_{1}\right|^{b_{2}}=0,\left|w_{1}\right|^{a_{2}}=0, r_{1}=m_{1}=0$ and the following equation holds in $F_{2}$ :

$$
w_{2}=w_{1} w_{2} \theta_{1}\left(a_{1}^{r_{2}}\right)\left(w_{1}\right) \text {. }
$$

b) $s_{2}$ is odd, $n_{1}=\left|w_{1}\right|^{b_{2}}=0$, either $n_{2}=\left|w_{2}\right|^{b_{2}}=0$ or $r_{1}=m_{1}=0$ and the following equation holds in $F_{2}$ :

$$
w_{2}=w_{1} \theta_{1}\left(a_{1}^{r_{1}}\right)\left(w_{2}\right) \theta_{1}\left(a_{1}^{r_{1}+r_{2}} b_{1}\right)\left(w_{1}\right)
$$

Even though we have not classified the maps from $K$ to $F_{2}(K)$, that is we don't know for which $r_{1}, r_{2}, s_{2} \in \mathbb{Z}$ and $w_{1}, w_{2} \in F_{2}$ a map from $K$ to $F_{2}(K)$ exists such that

$$
\begin{aligned}
\pi_{1}\left(K, p_{0}\right) & \longrightarrow F_{2} \rtimes_{\theta_{1}} \pi_{1}(K) \\
\alpha & \longmapsto w_{1} a_{1}^{r_{1}} \\
\beta & \longmapsto w_{2} a_{1}^{r_{2}} b_{1}^{s_{2}}
\end{aligned}
$$

is its induced map, it is possible to compute the Nielsen number assuming that we have a map.

Theorem 4.1.7. Let $\phi: K \multimap K$ be a 2-valued map and $\hat{\Phi}: K \rightarrow F_{2}(K)$ its lift, then there are words $w_{1}$ and $w_{2}$ in the free group $F_{2}$ and integers $r_{1}, r_{2}, s_{2} \in \mathbb{Z}$ such that

$$
\begin{aligned}
\hat{\Phi}_{\#}: \pi_{1}\left(K, p_{0}\right) & \longrightarrow F_{2} \rtimes_{\theta_{1}} \pi_{1}(K) \\
\alpha & \longmapsto \hat{\alpha}=w_{1} a_{1}^{r_{1}} \\
\beta & \longmapsto \hat{\beta}=w_{2} a_{1}^{r_{2}} b_{1}^{s_{2}} .
\end{aligned}
$$

Then the Nielsen number of $\phi$ is given by

$N(\phi)=\left\{\begin{array}{ll}\left|\left(s_{2}-1\right) r_{1}\right|+\left|\left(s_{2}+n_{2}-1\right)\left(m_{1}+r_{1}\right)\right| & s_{2}, n_{2} \text { odd and } r_{1}, m_{1}+r_{1} \neq 0, \\ \left|s_{2}-1\right|+\left|\left(s_{2}+n_{2}-1\right)\left(m_{1}+r_{1}\right)\right| & n_{2} \text { odd, } m_{1}+r_{1} \neq 0 \text { and if } s_{2} \text { odd, then } r_{1}=0, \\ \left|\left(s_{2}-1\right) r_{1}\right|+\left|\left(s_{2}+n_{2}-1\right)\right| & s_{2} \text { odd, } r_{1} \neq 0 \text { and if } n_{2} \text { odd, then } m_{1}=-r_{1}, \\ \left|s_{2}-1\right|+\left|\left(s_{2}+n_{2}-1\right)\right| & \text { otherwise. }\end{array}\right\}$

Proof. Since the Nielsen number of $\hat{\Phi}$ is given by $N(\hat{\Phi})=N\left(f_{1}\right)+N\left(f_{2}\right)$, (see Proposition 3.2.1) we compute the Nielsen number of $f_{1}$ and $f_{2}$. Start by the Nielsen number of $f_{1}$.

$$
N\left(f_{1}\right)= \begin{cases}\left|\left(s_{2}-1\right) r_{1}\right| & \text { if } s_{2} \text { is odd and } r_{1} \neq 0 \\ \left|s_{2}-1\right| & \text { otherwise. }\end{cases}
$$

Computing the Nielsen number of $f_{2}$ we obtain the following,

$$
N\left(f_{2}\right)=\left\{\begin{array}{cc}
\left|\left(s_{2}+n_{2}-1\right)\left(m_{1}+r_{1}\right)\right| & \text { if } n_{2}+s_{2} \text { is odd and } m_{1}+r_{1} \neq 0 \\
\left|\left(s_{2}+n_{2}-1\right)\right| & \text { otherwise }
\end{array}\right.
$$

Theorem 4.1.8. Let $\phi: K \multimap K$ be a 2-valued map with Nielsen number zero. Then the lift of the correspondent map $\hat{\Phi}: K \rightarrow F_{2}(K)$ induces a homomorphism

$$
\begin{aligned}
\hat{\Phi}_{\#}: \pi_{1}\left(K, p_{0}\right) & \longrightarrow F_{2} \rtimes_{\theta_{1}} \pi_{1}(K) \\
\alpha & \longmapsto \hat{\alpha}=w_{1} a_{1}^{r_{1}} \\
\beta & \longmapsto \hat{\beta}=w_{2} a_{1}^{r_{2}} b_{1},
\end{aligned}
$$

where $w_{1}$ and $w_{2}$ are wordsin the free group $F_{2}$ and $r_{1}, r_{2} \in \mathbb{Z}$ such that $n_{1}=\left|w_{1}\right|^{b_{2}}=0, n_{2}=$ 
$\left|w_{2}\right|^{b_{2}}=0$ and the Equation (4.4) holds in $F_{2}$, i.e.

$$
w_{2}=w_{1} \theta_{1}\left(a_{1}^{r_{1}}\right)\left(w_{2}\right) \theta_{1}\left(a_{1}^{r_{1}+r_{2}} b_{1}\right)\left(w_{1}\right) .
$$

The converse also holds, that is, if $w_{1}, w_{2} \in F_{2}$ with $n_{1}=\left|w_{1}\right|^{b_{2}}=0, n_{2}=\left|w_{2}\right|^{b_{2}}=0$ such that Equation (4.4) holds, then there is a 2-valued map $\phi: K \multimap K$ with Nielsen number zero such that the induced homomorphism $\hat{\Phi}_{\#}$ is given as in (4.6).

Proof. From Theorem 4.1.6 it follows that

$$
\begin{aligned}
\hat{\Phi}_{\#}: \pi_{1}\left(K, p_{0}\right) & \longrightarrow F_{2} \rtimes_{\theta_{1}} \pi_{1}(K) \\
\alpha & \longmapsto \hat{\alpha}=w_{1} a_{1}^{r_{1}} \\
\beta & \longmapsto \hat{\beta}=w_{2} a_{1}^{r_{2}} b_{1}^{s_{2}} .
\end{aligned}
$$

If we set the Nielsen number of $\hat{\Phi}$ zero, Theorem 4.1 .7 implies that $\left|s_{2}-1\right|=0$, hence $s_{2}=1$ is odd. Also $\left|\left(s_{2}+n_{2}-1\right)\right|=0$, which implies that $n_{2}=0$. Therefore, since $s_{2}$ is odd, item $\mathrm{b}$ ) of Theorem 4.1.6 holds, i.e. $n_{1}=\left|w_{1}\right|^{b_{2}}=0$, either $n_{2}=\left|w_{2}\right|^{b_{2}}=0$ or $r_{1}=m_{1}=0$ and equation (4.4) holds in $F_{2}$, which is

$$
w_{2}=w_{1} \theta_{1}\left(a_{1}^{r_{1}}\right)\left(w_{2}\right) \theta_{1}\left(a_{1}^{r_{1}+r_{2}} b_{1}\right)\left(w_{1}\right) .
$$

Since $n_{2}=0$ already, the integers $r_{1}$ and $m_{1}$ can be different from zero.

For the converse note that if Equation (4.4) holds, then by Proposition 4.1.1 it holds that $\hat{\alpha} \hat{\beta} \hat{\alpha}=\hat{\beta}$. Thus

$$
\begin{aligned}
\pi_{1}\left(K, p_{0}\right) & \longrightarrow F_{2} \rtimes_{\theta_{1}} \pi_{1}(K) \\
\alpha & \longmapsto \hat{\alpha}=w_{1} a_{1}^{r_{1}} \\
\beta & \longmapsto \hat{\beta}=w_{2} a_{1}^{r_{2}} b_{1},
\end{aligned}
$$

is a homomorphism and there is a 2 -valued map $\phi: K \multimap K$ such that the lift of its correspondent map $\hat{\Phi}: K \rightarrow F_{2}(K)$ induces this homomorphism. By Theorem 4.1.7 the Nielsen number of $\phi$ is zero.

Therefore, in order to classify the homotopy classes of split 2-valued maps on the Klein bottle with Nielsen number zero we need to solve the equation (4.4) in $F_{2}\left(a_{2}, b_{2}\right)$, where $\left|w_{2}\right|^{b_{2}}=0$.

\subsection{Solutions of an equation in the free group}

This section is dedicated in solving equation (4.4), i.e.

$$
w_{2}=w_{1} \theta_{1}\left(a_{1}^{r_{1}}\right)\left(w_{2}\right) \theta_{1}\left(a_{1}^{r_{1}+r_{2}} b_{1}\right)\left(w_{1}\right)
$$

in the subgroup $H=\left\{w \in F_{2}\left(a_{2}, b_{2}\right):|w|^{b_{2}}=0\right\}$ of $F_{2}\left(a_{2}, b_{2}\right)$ for arbitrary $r_{1}, r_{2} \in \mathbb{Z}$.

Lemma 4.2.1. Let $w_{1}, w_{2} \in F_{2}$ satisfy equation (4.4), that is

$$
w_{2}=w_{1} \theta_{1}\left(a_{1}^{r_{1}}\right)\left(w_{2}\right) \theta_{1}\left(a_{1}^{r_{1}+r_{2}} b_{1}\right)\left(w_{1}\right),
$$

then

$$
w_{1}=\left(w_{2} \theta_{1}\left(a_{1}^{r_{2}} b_{1}\right)\left(w_{2}\right)\right)^{-1} w_{1} \theta_{1}\left(a_{1}^{r_{1}}\right)\left(w_{2} \theta_{1}\left(a_{1}^{r_{2}} b_{1}\right)\left(w_{2}\right)\right) .
$$

Proof. Apply $\theta_{1}\left(a_{1}^{r_{1}+r_{2}} b_{1}\right)$ on the following equation

$$
\theta_{1}\left(a_{1}^{r_{1}+r_{2}} b_{1}\right)\left(w_{1}\right)=\theta_{1}\left(a_{1}^{r_{1}}\right)\left(w_{2}^{-1}\right) w_{1}^{-1} w_{2},
$$


which is a equivalent to equation (4.4). Then, since $\theta_{1}$ is a homomorphism which is inverse to itself and from the relations in the Klein bottle $\left\langle a_{1}, b_{1}, a_{1} b_{1} a_{1}=b_{1}\right\rangle$ it follows

$$
\begin{aligned}
w_{1} & =\theta_{1}^{2}\left(a_{1}^{r_{1}+r_{2}} b_{1}\right)\left(w_{1}\right) \\
& =\theta_{1}\left(a_{1}^{r_{1}+r_{2}} b_{1}\right)\left(\theta_{1}\left(a_{1}^{r_{1}}\right)\left(w_{2}^{-1}\right) w_{1}^{-1} w_{2}\right) \\
& =\theta_{1}\left(a_{1}^{r_{2}} b_{1}\right)\left(w_{2}^{-1}\right) \theta_{1}\left(a_{1}^{r_{1}+r_{2}} b_{1}\right)\left(w_{1}^{-1}\right) \theta_{1}\left(a_{1}^{r_{1}+r_{2}} b_{1}\right)\left(w_{2}\right) .
\end{aligned}
$$

Since from equation equation (4.4) we know that

$$
\theta_{1}\left(a_{1}^{r_{1}+r_{2}} b_{1}\right)\left(w_{1}^{-1}\right)=w_{2}^{-1} w_{1} \theta_{1}\left(a_{1}^{r_{1}}\right)\left(w_{2}\right) .
$$

it follows

$$
\begin{aligned}
w_{1} & =\theta_{1}\left(a_{1}^{r_{2}} b_{1}\right)\left(w_{2}^{-1}\right)\left[w_{2}^{-1} w_{1} \theta_{1}\left(a_{1}^{r_{1}}\right)\left(w_{2}\right)\right] \theta_{1}\left(a_{1}^{r_{1}+r_{2}} b_{1}\right)\left(w_{2}\right) \\
& =\left[w_{2} \theta_{1}\left(a_{1}^{r_{2}} b_{1}\right)\left(w_{2}\right)\right]^{-1} w_{1}\left[\theta_{1}\left(a_{1}^{r_{1}}\right) w_{2} \theta_{1}\left(a_{1}^{r_{2}} b_{1}\right)\left(w_{2}\right)\right] .
\end{aligned}
$$

Recall that, for every $r_{1}$ and every $\hat{t} \in \mathbb{Z}$ it holds that

$$
\begin{aligned}
\theta_{1}\left(a_{1}^{r_{1}}\right): F_{2} & \longrightarrow F_{2} \\
a_{2} & \longmapsto a_{2} \\
b_{2} & \longmapsto a_{2}^{2 r_{1}} b_{2} \\
\theta_{1}\left(a_{1}^{\hat{t}} b_{1}\right): F_{2} & \longrightarrow F_{2} \\
a_{2} & \longmapsto a_{2}^{-1} \\
b_{2} & \longmapsto a_{2}^{2(\hat{t})+1} b_{2} a_{2} .
\end{aligned}
$$

To simplify the notation, we denote for every $r_{1} \in \mathbb{Z}$ and $t=2 \hat{t}+1$, where $\hat{t} \in \mathbb{Z}$ the automorphisms

$$
\begin{aligned}
\eta & =\theta_{1}\left(a_{1}^{r_{1}}\right), \\
\mu_{t} & =\theta_{1}\left(a_{1}^{\hat{t}} b_{1}\right) .
\end{aligned}
$$

Three automorphisms that are used frequently in the next pages are $\eta, \mu_{2 r_{1}+1}$ and $\mu_{\left(r_{1}+r_{2}\right)+1}$. Since $r_{1}$ and $r_{2}$ are arbitrary elements in $\mathbb{Z}$ and $\mu_{2 r_{1}+1}$ is different to $\mu_{2\left(r_{1}+r_{2}\right)+1}$ whenever $r_{2} \neq 1$, we define and study $\mu_{t}$ for any odd $t \in \mathbb{Z}$.

An important fact to note is that both $\eta$ and $\mu_{t}$ take words of $H$ into $H$, since $|w|^{b_{2}}=|\eta(w)|^{b_{2}}=$ $\left|\mu_{t}(w)\right|^{b_{2}}$. Therefore we can consider their restrictions to $H$, which we will denote, abusing the notation, by $\eta$ and $\mu_{t}$. From Lemma 2.1.4 it follows

a) $\theta_{1}\left(a_{1}^{-r_{1}}\right) \circ \theta_{1}\left(a_{1}^{r_{1}+r_{2}} b_{1}\right)=\theta_{1}\left(a_{1}^{r_{1}+r_{2}} b_{1}\right) \circ \theta_{1}\left(a_{1}^{r_{1}}\right)=\theta_{1}\left(a_{1}^{r_{2}} b_{1}\right)$

b) $\theta_{1}\left(a_{1}^{\hat{t}} b_{1}\right)^{2}=i d$, for all $\hat{t} \in \mathbb{Z}$.

and therefore for every odd $t \in \mathbb{Z}$

$$
\begin{aligned}
\mu_{t}^{2} & =i d_{F_{2}} \\
\eta^{-1} \circ \mu_{t} & =\mu_{t} \circ \eta=\mu_{t-2 r_{1}}, \\
\eta \circ \mu_{t} & =\mu_{t} \circ \eta^{-1}=\mu_{t+2 r_{1}} .
\end{aligned}
$$

Now (4.4) can be written as follows

$$
w_{2}=w_{1} \eta\left(w_{2}\right) \mu_{r}\left(w_{1}\right),
$$


where $r=2\left(r_{1}+r_{2}\right)+1$ This is equivalent to

$$
\mu_{r}\left(w_{1}\right)=\eta\left(w_{2}^{-1}\right) w_{1}^{-1} w_{2} .
$$

Writing Lemma 4.2.1 with this notation gives us the following statement.

Corollary 4.2.1.1. Let $w_{1}, w_{2} \in F_{2}$ satisfy equation (4.11), i.e.

$$
\mu_{r}\left(w_{1}\right)=\eta\left(w_{2}^{-1}\right) w_{1}^{-1} w_{2},
$$

then

$$
w_{1}=\left(w_{2} \mu_{2 r_{2}+1}\left(w_{2}\right)\right)^{-1} w_{1} \eta\left(w_{2} \mu_{2 r_{2}+1}\left(w_{2}\right)\right) .
$$

Therefore for $w_{1}$ and $w_{2}$ to satisfy equation (4.11) equation (4.12) has to hold. Hence the idea is to find the solutions of equation (4.12) first and then study equation (4.11), restricting to the solutions obtained.

One family that satisfies equation (4.12) in a very straigthforward way is $w_{1}$ and $w_{2}$, such that $w_{1}$ is arbitrary and for $w_{2}$ it holds that

$$
w_{2} \mu_{2 r_{2}+1}\left(w_{2}\right)=1 \text {. }
$$

Suppose that this is the case, then the following Proposition holds.

Proposition 4.2.2. Let $w_{2} \in F_{2}$ with $\left(\mu_{2 r_{2}+1}\right)\left(w_{2}\right)=w_{2}^{-1}$.

If $w_{1}$ and $w_{2}$ satisfy equation (4.11), i.e.

$$
\mu_{r}\left(w_{1}\right)=\eta\left(w_{2}^{-1}\right) w_{1}^{-1} w_{2},
$$

then there is a word $u$ in $H$ with $\mu_{r}(u)=u^{-1}$ such that $w_{1}=w_{2} u$.

Conversely, if $w_{1}=w_{2} u$ for any word $u \in H$ with $\mu_{r}(u)=u^{-1}$, then

$$
\mu_{r}\left(w_{1}\right)=\eta\left(w_{2}^{-1}\right) w_{1}^{-1} w_{2} .
$$

Proof. If we apply $\mu_{r}$ to equation (4.4) we get, using that $\mu_{2 r_{2}+1}\left(w_{2}^{-1}\right)=w_{2}$, that

$$
w_{1}=\mu_{r}^{2}\left(w_{1}\right)=\left(\mu_{r} \circ \eta\right)\left(w_{2}^{-1}\right) \mu_{r}\left(w_{1}^{-1}\right) \mu_{r}\left(w_{2}\right)=\mu_{2 r_{2}+1}\left(w_{2}^{-1}\right) \mu_{r}\left(w_{1}^{-1}\right) \mu_{r}\left(w_{2}\right)=w_{2} \mu_{r}\left(w_{1}^{-1} w_{2}\right) .
$$

Therefore

$$
\mu\left(w_{1}^{-1} w_{2}\right)=w_{2}^{-1} w_{1}=\left(w_{1}^{-1} w_{2}\right)^{-1}
$$

and consequently

$$
\mu\left(w_{2}^{-1} w_{1}\right)=\left(w_{2}^{-1} w_{1}\right)^{-1}
$$

Set $u=w_{2}^{-1} w_{1} \in H$. Conversely, let $u, w_{1} \in H$ be words such that $\mu(u)=u^{-1}$ and $w_{1}=w_{2} u$. Then, using $\mu_{2 r_{2}+1}\left(w_{2}\right)=w_{2}^{-1}$, we get that

$$
\begin{aligned}
\eta\left(w_{2}^{-1}\right) w_{1}^{-1} w_{2} & =\eta\left(\mu_{2 r_{2}+1}\left(w_{2}\right)\right)\left(w_{2} u\right)^{-1} w_{2} \\
& =\mu_{r}\left(w_{2}\right) u^{-1} w_{2}^{-1} w_{2} \\
& =\mu_{r}\left(w_{2}\right) u^{-1} \\
& =\mu_{r}\left(w_{2}\right) \mu_{r}(u) \\
& =\mu_{r}\left(w_{1}\right) .
\end{aligned}
$$


Therefore in case that $\left(\mu_{2 r_{2}+1}\right)\left(w_{2}\right)=w_{2}^{-1}$ it is sufficient to solve the following equation in $H$ for $t$ equal to $2 r_{1}+1$ and $2\left(r_{1}+r_{2}\right)+1$

$$
\mu_{t}(w)=w^{-1}
$$

Section 4.2.2 is dedicated in solving equation (4.13) for every odd $t$.

To find the other solutions of Equation (4.12) suppose that $v=w_{2} \mu_{2 r_{2}+1}\left(w_{2}\right)$ is not the empty word. So we write equation (4.12), i.e.

$$
w_{1}=\left(w_{2} \mu_{2 r_{2}+1}\left(w_{2}\right)\right)^{-1} w_{1} \eta\left(w_{2} \mu_{2 r_{2}+1}\left(w_{2}\right)\right),
$$

as a system of two equations

$$
\begin{aligned}
w_{1} & =v^{-1} w_{1} \eta(v) \\
v & =w_{2} \mu_{2 r_{2}+1}\left(w_{2}\right),
\end{aligned}
$$

where $v, w_{1}, w_{2} \in H$ with $v \neq 1$. Equation (4.14) is equivalent to

$$
\eta(v)=w_{1}^{-1} v w_{1}
$$

hence, to solve this equation we seek the words in $H$ that are taken to a conjugate by $\eta$. Also, applying $\mu_{2 r_{2}+1}$ on equation (4.15) and using that $\mu_{2 r_{2}+1}\left(w_{2}\right)=w_{2}^{-1} v$ implies

$$
\mu_{2 r_{2}+1}(v)=\mu_{2 r_{2}+1}\left(w_{2}\right) w_{2}=w_{2}^{-1} v w_{2} .
$$

Hence equation (4.12), and consequently equation (4.11) implies that the following system of equations in $H$ holds,

$$
\begin{aligned}
\eta(v) & =w_{1}^{-1} v w_{1}, \\
\mu_{2 r_{2}+1}(v) & =w_{2}^{-1} v w_{2},
\end{aligned}
$$

where $v \neq 1$.

Lemma 4.2.3. Let $w_{2} \in F_{2}$ with $\left(\mu_{2 r_{2}+1}\right)\left(w_{2}\right) w_{2} \neq 1$ and let $w_{1}$ and $w_{2}$ satisfy equation (4.11), i.e.

$$
\mu_{r}\left(w_{1}\right)=\eta\left(w_{2}^{-1}\right) w_{1}^{-1} w_{2} .
$$

Let also $v=\left(\mu_{2 r_{2}+1}\right)\left(w_{2}\right) w_{2}$. Then the following system of equation holds in $H$,

$$
\begin{aligned}
\eta(v) & =w_{1}^{-1} v w_{1}, \\
\mu_{2 r_{2}+1}(v) & =w_{2}^{-1} v w_{2}, .
\end{aligned}
$$

Hence solving this system of equations for $w_{1}, w_{2}$ and $v$ in $H$ provides necessary conditions for $w_{1}$ and $w_{2}$ to satisfy the equation $(4.11)$, in case $w_{2}\left(\mu_{2 r_{2}+1}\right)\left(w_{2}\right)$ is not empty. We compute the solutions of this system in section 4.2.3 and actually show that these conditions are indeed sufficient.

To proceed as above indicated it is necessary to do some technical computations. We write a base for $H$ and compute how $\mu_{r}, \eta$ and $\mu_{2 r_{2}+1}=\mu_{r} \circ \eta$ act on elements of this base.

\subsubsection{Analyzing $\mu_{t}$ and $\eta$ in $H$}

Since both $w_{1}$ and $w_{2}$ are contained in the subgroup $H=\left\{w \in F_{2}:|w|^{b_{2}}=0\right\}$ of $F_{2}$ we work on $H$ and is essential to know a base for $H$, i.e. a generating set $\mathcal{B}$ of $H$, such that every word in $H$ can be written uniquely as a product of elements of $\mathcal{B}$. To do so we use the Reidermeister-Schreier Method (see for example [LS15] or [MKS04]). 
Proposition 4.2.4. Let $\mathcal{B}=\left\{C_{n}: n \in \mathbb{Z}\right\}$, where $C_{n}=b_{2}^{n} a_{2} b_{2}^{-n}$. Then $H=\left\{w \in F_{2}:|w|^{b_{2}}=0\right\}$ is the free group on the letters $C_{n}$, where $n \in \mathbb{Z}$.

Proof. Consider the homomorphism from $F_{2}$ to $\mathbb{Z}$ given by

$$
\begin{aligned}
F_{2} \longrightarrow \mathbb{Z} \\
a_{2} \longmapsto 0, \\
b_{2} \longmapsto 1 .
\end{aligned}
$$

Then $H$ is the kernel of this surjective map, thus $F_{2} \backslash H$ is isomorfic to $\mathbb{Z}$. As Schreier transversal we choose the set $T=\left\{b_{2}^{n}: n \in \mathbb{Z}\right\}$, which is a set of representatives of the right cosets of $H$ in $F_{2}$ such that every initial segment of an element of $T$ itself belongs to $T$. It holds that $H b_{2}^{n} a_{2}=H b_{2}^{n}$, this is $b_{2}^{n}$ is the representative of $b_{2}^{n} a_{2}$. Of course $b_{2}^{n}$ is already a representative. Therefore, after the Reidermeister-Schreier method it follows that $H$ has the following representation:

$$
H=\left\langle b_{2}^{n} a_{2} b_{2}^{-n}: n \in \mathbb{Z}\right\rangle .
$$

It is useful to know how $\mu_{t}$ and $\eta$ act on the words $C_{n}=b_{2}^{n} a_{2} b_{2}^{-n}$. But before we do this, to facilitate the notation, set for every integer $t, n \in \mathbb{Z}$

$$
\zeta_{n}(t)=\left\{\begin{array}{ll}
C_{0}^{t} C_{1}^{t} \cdot \ldots \cdot C_{n-1}^{t} & \text { if } n>0 \\
C_{-1}^{-t} \cdot \ldots \cdot C_{n+1}^{-t} & \text { if } n<-1 \\
1 & \text { if } n=0 \text { or } n=-1 .
\end{array}\right\}
$$

Note that if $t=0$, then $\zeta_{n}(t)=0$. To facilitate we use the notation $\zeta_{n}^{-1}(k)$ instead of $\left(\zeta_{n}(k)\right)^{-1}$. Note the following technical results for $C_{n}$ and $\zeta_{n}(t)$.

Lemma 4.2.5. The following formulas hold.

i) For every integer $n \in \mathbb{Z}$

$$
\begin{aligned}
& b_{2} C_{n} b_{2}^{-1}=C_{n+1} \\
& b_{2}^{-1} C_{n} b_{2}=C_{n-1} .
\end{aligned}
$$

ii) For every $t \in \mathbb{Z}$ and $n \in \mathbb{Z} \backslash\{-1\}$,

$$
b_{2} \zeta_{n}(t) b_{2}^{-1}=C_{0}^{-t} \zeta_{n+1}(t) .
$$

iii) For every $t \in \mathbb{Z}$ and $n \in \mathbb{Z} \backslash\{0\}$

$$
b_{2}^{-1} \zeta_{n}(t) b_{2}=C_{-1}^{t} \zeta_{n-1}(t)
$$

Proof. Item i) follows from

$$
b_{2} C_{n} b_{2}^{-1}=b_{2}\left(b_{2}^{n} a_{2} b_{2}^{-n}\right) b^{-1}=b_{2}^{n+1} a_{2} b_{2}^{-(n+1)}=C_{n+1} .
$$

For ii) separate in three cases. If $n>0$, then

$$
b_{2} \zeta_{n}(t) b_{2}^{-1}=b_{2} C_{0}^{t} \cdot \ldots \cdot C_{n-1}^{t} b_{2}^{-1}=C_{1}^{t} \cdot \ldots \cdot C_{n}^{t}=C_{0}^{-t} C_{0}^{t} \cdot \ldots \cdot C_{n}^{t}=C_{0}^{-t} \zeta_{n+1}(t) .
$$

If $n=0$, then

$$
b_{2} \zeta_{n}(t) b_{2}^{-1}=b_{2} \zeta_{0}(t) b_{2}^{-1}=1=C_{0}^{-t} C_{0}^{t}=C_{0}^{-t} \zeta_{1}(t)=C_{0}^{-t} \zeta_{n+1}(t) .
$$


If $n<-1$, then

$$
b_{2} \zeta_{n}(t) b_{2}^{-1}=b_{2} C_{-1}^{-t} C_{-2}^{-t} \cdot \ldots \cdot C_{n+1}^{-t} b_{2}^{-1}=C_{0}^{-t} C_{-1}^{-t} \cdot \ldots \cdot C_{n+2}^{-t}=C_{0}^{-t} \zeta_{n+1}(t) .
$$

Item iii) follows straightforwardly from ii). Indeed we know from ii) that

$$
\zeta_{n}(t)=b_{2}^{-1} C_{0}^{-t} \zeta_{n+1}(t) b_{2}=C_{-1}^{-t} b_{2}^{-1} \zeta_{n+1}(t) b_{2} .
$$

for every $n \in \mathbb{Z} \backslash\{-1\}$. Therefore

$$
b_{2}^{-1} \zeta_{n+1}(t) b_{2}=C_{-1}^{t} \zeta_{n}(t)
$$

Proposition 4.2.6. Let $n$ be any integer, $t$ a non-zero integer, then

$$
\mu_{t}\left(C_{n}\right)=\left[C_{0}^{-1} \zeta_{n}(t+1)\right] C_{n}^{-1}\left[\zeta_{n}^{-1}(t+1) C_{0}\right]
$$

Proof. We show this by induction. Start noting, that for all $t \in \mathbb{Z}$ it holds that

$$
\mu_{t}\left(C_{0}\right)=\mu_{t}\left(a_{2}\right)=a_{2}^{-1}=C_{0}^{-1}=C_{0}^{-1} C_{0}^{-1} C_{0}=\left[C_{0}^{-1} \zeta_{0}(t+1)\right] C_{0}^{-1}\left[\zeta_{0}^{-1}(t+1) C_{0}\right] .
$$

and

$$
\begin{aligned}
\mu_{t}\left(C_{-1}\right) & =\mu_{t}\left(b_{2}^{-1} C_{0} b_{2}\right) \\
& =\left[C_{0}^{-1} b_{2}^{-1} C_{0}^{-t}\right] C_{0}^{-1}\left[C_{0}^{t} b_{2} C_{0}\right] \\
& =C_{0}^{-1}\left[b_{2}^{-1} C_{0}^{-1} b_{2}\right] C_{0} \\
& =C_{0}^{-1} C_{-1}^{-1} b_{2} C_{0} \\
& =\left[C_{0}^{-1} \zeta_{-1}(t+1)\right] C_{-1}^{-1}\left[\zeta_{-1}^{-1}(t+1) C_{0}\right] .
\end{aligned}
$$

Suppose that for $n \geq 0$ the hypothesis holds, then, using Lemma 4.2.5

$$
\begin{aligned}
\mu_{t}\left(C_{n+1}\right) & =\mu_{t}\left(b_{2} C_{n} b_{2}^{-1}\right) \\
& =\left[C_{0}^{t} b_{2} C_{0}\right]\left[C_{0}^{-1} \zeta_{n}(t+1) C_{n}^{-1} \zeta_{n}^{-1}(t+1) C_{0}\right]\left[C_{0}^{-1} b_{2}^{-1} C_{0}^{-t}\right] \\
& =C_{0}^{t}\left[b_{2} \zeta_{n}(t+1) b_{2}^{-1}\right]\left[b_{2} C_{n}^{-1} b_{2}^{-1}\right]\left[b_{2} \zeta_{n}^{-1}(t+1) b_{2}^{-1}\right] C_{0}^{-t} \\
& =C_{0}^{t}\left[C_{0}^{-t-1} \zeta_{n+1}(t+1)\right] C_{n+1}^{-1}\left[\zeta_{n+1}^{-1}(t+1) C_{0}^{t+1}\right] C_{0}^{-t} \\
& =\left[C_{0}^{-1} \zeta_{n+1}(t+1)\right] C_{n+1}^{-1}\left[\zeta_{n+1}^{-1}(t+1) C_{0}\right] .
\end{aligned}
$$

Similarly, if we suppose that the hypothesis holds for $n \leq-1$, then

$$
\begin{aligned}
\mu_{t}\left(C_{n-1}\right) & =\mu_{t}\left(b_{2}^{-1} C_{n} b_{2}\right) \\
& =\left(C_{0}^{-1} b_{2}^{-1} C_{0}^{-t}\right)\left(C_{0}^{-1} \zeta_{n}(t+1) C_{n}^{-1} \zeta_{n}^{-1}(t+1) C_{0}\right)\left(C_{0}^{t} b_{2} C_{0}\right) \\
& =C_{0}^{-1}\left(b_{2}^{-1} C_{0}^{-t-1} b_{2}\right)\left(b_{2}^{-1} \zeta_{n}(t+1) b_{2}\right)\left(b_{2}^{-1} C_{n}^{-1} b_{2}\right)\left(b_{2}^{-1} \zeta_{n}^{-1}(t+1) b_{2}\right)\left(b_{2}^{-1} C_{0}^{t+1} b_{2}\right) C_{0} \\
& =C_{0}^{-1}\left(C_{-1}^{-t-1}\right)\left(C_{-1}^{t+1} \zeta_{n-1}(t+1)\right)\left(C_{n-1}^{-1}\right)\left(\zeta_{n-1}^{-1}(t+1) C_{-1}^{-t-1}\right)\left(C_{-1}^{t+1}\right) C_{0} \\
& =\left(C_{0}^{-1} \zeta_{n-1}(t+1)\right) C_{n-1}^{-1}\left(\zeta_{n-1}^{-1}(t+1)\right) C_{0} .
\end{aligned}
$$

Applying $\eta$ on an element of the base we get

Lemma 4.2.7. It holds, for every $n \in \mathbb{Z}$ that

$$
\eta\left(C_{n}\right)=\zeta_{n}\left(2 r_{1}\right) C_{n} \zeta_{n}^{-1}\left(2 r_{1}\right)
$$


Proof. We show this by induction on $n$. Let $n=0$, then

$$
\eta\left(C_{0}\right)=\eta\left(a_{2}\right)=a_{2}=C_{0}=\zeta_{0}\left(2 r_{1}\right) C_{0} \zeta_{0}^{-1}\left(2 r_{1}\right)
$$

and for $n=-1$

$$
\eta\left(C_{-1}\right)=\eta\left(b_{2}^{-1} C_{0} b_{2}\right)=\left(b_{2}^{-1} C_{0}^{-2 r_{1}}\right) C_{0}\left(C_{0}^{2 r_{1}} b_{2}\right)=b_{2}^{-1} C_{0} b_{2}=C_{-1} .
$$

Suppose that the hypothesis holds for a fixed $n \geq 0$, then

$$
\begin{aligned}
\eta\left(C_{n+1}\right) & =\eta\left(b_{2} C_{n} b_{2}^{-1}\right) \\
& =\left(C_{0}^{2 r_{1}} b_{2}\right)\left(\zeta_{n}\left(2 r_{1}\right) C_{n} \zeta_{n}^{-1}\left(2 r_{1}\right)\right)\left(b_{2}^{-1} C_{0}^{-2 r_{1}}\right) \\
& =C_{0}^{2 r_{1}}\left(b_{2} \zeta_{n}\left(2 r_{1}\right) b_{2}^{-1}\right)\left(b_{2} C_{n} b_{2}^{-1}\right)\left(b_{2} \zeta_{n}^{-1}\left(2 r_{1}\right) b_{2}^{-1}\right) C_{0}^{-2 r_{1}} \\
& =C_{0}^{2 r_{1}}\left(C_{0}^{-2 r_{1}} \zeta_{n+1}\left(2 r_{1}\right)\right) C_{n+1}\left(\zeta_{n+1}^{-1}\left(2 r_{1}\right) C_{0}^{2 r_{1}}\right) C_{0}^{-2 r_{1}} \\
& =\zeta_{n+1}\left(2 r_{1}\right) C_{n+1} \zeta_{n+1}^{-1}\left(2 r_{1}\right) .
\end{aligned}
$$

Also, if we suppose that the hypothesis holds for a fixed $n \leq-1$, then

$$
\begin{aligned}
\eta\left(C_{n-1}\right) & =\eta\left(b_{2}^{-1} C_{n} b_{2}\right) \\
& =\left(b_{2}^{-1} C_{0}^{-2 r_{1}}\right)\left(\zeta_{n}\left(2 r_{1}\right) C_{n} \zeta_{n}^{-1}\left(2 r_{1}\right)\right)\left(C_{0}^{2 r_{1}} b_{2}\right) \\
& =\left(b_{2}^{-1} C_{0}^{-2 r_{1}} b_{2}\right)\left(b_{2}^{-1} \zeta_{n}\left(2 r_{1}\right) b_{2}^{-1}\right)\left(b_{2} C_{n} b_{2}^{-1}\right)\left(b_{2} \zeta_{n}^{-1}\left(2 r_{1}\right) b_{2}\right)\left(b_{2}^{-1} C_{0}^{2 r_{1}} b_{2}\right) \\
& \left.=C_{-1}^{-2 r_{1}} C_{-1}^{2 r_{1}} \zeta_{n-1}\left(2 r_{1}\right) C_{n-1} \zeta_{n-1}^{-1}\left(2 r_{1}\right) C_{-1}^{-2 r_{1}}\right) C_{-1}^{2 r_{1}} \\
& =\zeta_{n-1}\left(2 r_{1}\right) C_{n-1} \zeta_{n-1}^{-1}\left(2 r_{1}\right),
\end{aligned}
$$

which concludes the induction.

Another technical fact, needed in further computations is a computation of the images of $\zeta_{n}(t)$ is, under the maps $\eta$ and $\mu_{t_{1}}$. For this consider first the following statement.

Proposition 4.2.8. For $n \in \mathbb{Z} \backslash\{0,-1\}$ and every $t \in Z$ it holds

$$
\zeta_{n}(t)=\zeta_{n-\operatorname{sgn}(n)}(t) C_{n-\operatorname{sgn}(n)}^{\operatorname{sgn}(n) t} .
$$

Proof. By definition,

$$
\zeta_{n}(t)=\left\{\begin{array}{ll}
C_{0}^{t} C_{1}^{t} \cdot \ldots \cdot C_{n-1}^{t} & \text { if } n>0 \\
C_{-1}^{-t} \cdot \ldots \cdot C_{n+1}^{-t} & \text { if } n<-1 \\
1 & \text { if } n=0 \text { or } n=-1 .
\end{array}\right\}
$$

we show that

$$
\zeta_{n-\operatorname{sgn}(n)}(t)=\zeta_{n}(t) C_{n-\operatorname{sgn}(n)}^{-\operatorname{sgn}(n) t}
$$

To proof the statement we will separate into two cases, $n>0$ and $n<-1$. If $n>0$, then

$$
\begin{aligned}
\zeta_{n}(t) C_{n-\operatorname{sgn}(n)}^{-\operatorname{sgn}(n) t} & =\left(C_{0}^{t} C_{1}^{t} \cdot \ldots \cdot C_{n-1}^{t}\right) C_{n-1}^{-t} \\
& =\zeta_{n-1}(t) \\
& =\zeta_{n-\operatorname{sgn}(n)}(t) .
\end{aligned}
$$


If $n<-1$, then

$$
\begin{aligned}
\zeta_{n}(t) C_{n-\operatorname{sgn}(n)}^{-\operatorname{sgn}(n) t} & =\left(C_{-1}^{-t} \cdot \ldots \cdot C_{n+1}^{-t}\right) C_{n+1}^{t} \\
& =\zeta_{n+1}(t) \\
& =\zeta_{n-\operatorname{sgn}(n)}(t) .
\end{aligned}
$$

Proposition 4.2.9. For every $t \in \mathbb{Z}$ different to zero.

$$
\eta\left(\zeta_{n}(t)\right)=\zeta_{n}\left(t+2 r_{1}\right) \zeta_{n}^{-1}\left(2 r_{1}\right)
$$

Also, for every integer $n \in \mathbb{Z}$

$$
\mu_{t_{1}}\left(\zeta_{n}\left(t_{2}\right)\right)=C_{0}^{-1} \zeta_{n}\left(t_{1}-t_{2}+1\right) \zeta_{n}^{-1}\left(t_{1}+1\right) C_{0} .
$$

Proof. Both we prove by induction on $n$. Since the statement is for $n$ in $\mathbb{Z}$ we will show that if the hypothesis holds for $n-\operatorname{sgn}(n)$, then it holds for $n$. For $n=0$ and $n=-1$ both equations hold, as $\zeta_{0}(t)=\zeta_{-1}(t)=1$ for every $t \in \mathbb{Z}$. For the induction start with equation (4.19). Let $n$ be an integer in $\mathbb{Z} \backslash\{0,-1\}$ and suppose that the equation (4.19) holds for $n-\operatorname{sgn}(n)$, i.e.

$$
\eta\left(\zeta_{n-\operatorname{sgn}(n)}(t)\right)=\zeta_{n-\operatorname{sgn}(n)}\left(t+2 r_{1}\right) \zeta_{n-\operatorname{sgn}(n)}^{-1}\left(2 r_{1}\right) .
$$

Then it follows that

$$
\begin{aligned}
\eta\left(\zeta_{n}(t)\right) & =\eta\left(\zeta_{n-\operatorname{sgn}(n)}(t) C_{n-\operatorname{sgn}(n)}^{\operatorname{sgn}(n)(t)}\right) \\
& \left.=\zeta_{n-\operatorname{sgn}(n)}\left(t+2 r_{1}\right) \zeta_{n-\operatorname{sgn}(n)}^{-1}\left(2 r_{1}\right)\left(\zeta_{n-\operatorname{sgn}(n)}\left(2 r_{1}\right) C_{n-\operatorname{sgn}(n)}^{\operatorname{sgn}(n) t}\right) \zeta_{n-\operatorname{sgn}(n)}^{-1}\left(2 r_{1}\right)\right) \\
& \left.=\zeta_{n-\operatorname{sgn}(n)}\left(t+2 r_{1}\right) C_{n-\operatorname{sgn}(n)}^{\operatorname{sgn}(n) t} \zeta_{n-\operatorname{sgn}(n)}^{-1}\left(2 r_{1}\right)\right) \\
& =\left(\zeta_{n-\operatorname{sgn}(n)}\left(t+2 r_{1}\right) C_{n-\operatorname{sgn}(n)}^{\operatorname{sgn}(n)\left(+2 r_{1}\right)}\right)\left(C_{n-\operatorname{sgn}(n)}^{\operatorname{sgn}(n)\left(-2 r_{1}\right)} \zeta_{n-\operatorname{sgn}(n)}^{-1}\left(2 r_{1}\right)\right) \\
& =\zeta_{n}\left(t+2 r_{1}\right) \zeta_{n}^{-1}\left(2 r_{1}\right) .
\end{aligned}
$$

Now fix an integer $n$ in $\mathbb{Z} \backslash\{0,-1\}$ and suppose that the equation (4.20) holds for $n-\operatorname{sgn}(n)$, i.e.

$$
\mu_{t_{1}}\left(\zeta_{n-\operatorname{sgn}(n)}\left(t_{2}\right)\right)=C_{0}^{-1} \zeta_{n-\operatorname{sgn}(n)}\left(t_{1}-t_{2}+1\right) \zeta_{n-\operatorname{sgn}(n)}^{-1}\left(t_{1}+1\right) C_{0}
$$

Then it follows that

$$
\begin{aligned}
\mu_{t_{1}}\left(\zeta_{n}\left(t_{2}\right)\right) & =\mu_{t_{1}}\left(\zeta_{n-\operatorname{sgn}(n)}\left(t_{2}\right) C_{n-\operatorname{sgn}(n)}^{\operatorname{sgn}(n) t_{2}}\right) \\
& =\mu_{t_{1}}\left(\zeta_{n-\operatorname{sgn}(n)}\left(t_{2}\right)\right) \mu_{t_{1}}\left(C_{n-\operatorname{sgn}(n)}^{\operatorname{sgn}(n) t_{2}}\right) \\
& \left.=C_{0}^{-1} \zeta_{n-\operatorname{sgn}(n)}\left(t_{1}-t_{2}+1\right) C_{n-\operatorname{sgn}(n)}^{-\operatorname{sgn}(n) t_{2}} \zeta_{n-\operatorname{sgn}(n)}^{-1}\left(t_{1}+1\right) C_{0}\right) \\
& \left.=C_{0}^{-1}\left(\zeta_{n-\operatorname{sgn}(n)}\left(t_{1}-t_{2}+1\right) C_{n-\operatorname{sgn}(n)}^{\operatorname{sgn}(n)\left(t_{1}-t_{2}+1\right)}\right)\left(C_{n-\operatorname{sgn}(n)}^{-\operatorname{sgn}(n)\left(t_{1}+1\right)} \zeta_{n-\operatorname{sgn}(n)}^{-1}\left(t_{1}+1\right)\right) C_{0}\right) \\
& \left.=C_{0}^{-1} \zeta_{n}\left(t_{1}-t_{2}+1\right)\left(\zeta_{n-\operatorname{sgn}(n)}\left(t_{1}+1\right) C_{n-\operatorname{sgn}(n)}^{\operatorname{sgn}(n)\left(t_{1}+1\right)}\right)^{-1} C_{0}\right) \\
& =C_{0}^{-1} \zeta_{n}\left(t_{1}-t_{2}+1\right) \zeta_{n}^{-1}\left(t_{1}+1\right) C_{0} .
\end{aligned}
$$

Remember that $r=2 r_{1}+2 r_{2}+1$, then it follows directly 
Corollary 4.2.9.1. For every integer $n$ and $t$ it holds

$$
\begin{aligned}
\mu_{t}\left(\zeta_{n}(t+1)\right) & =C_{0}^{-1} \zeta_{n}^{-1}(t+1) C_{0} \\
\mu_{r}\left(\zeta_{n}\left(2 r_{1}\right)\right) & =C_{0}^{-1} \zeta_{n}\left(2 r_{2}+2\right) \zeta_{n}^{-1}(r+1) C_{0} .
\end{aligned}
$$

\subsubsection{Case $\mu_{2 r_{2}}\left(w_{2}\right)=w_{2}^{-1}$}

In this section we find all solutions of equation (4.13), i.e. for

$$
\mu_{t}(w)=w^{-1}
$$

where $t$ is odd. Recall that $\mu_{t}$ is defined in equation (4.7), for every odd $t=2 \hat{t}+1$ as

$$
\begin{aligned}
\mu_{t}=\theta_{1}\left(a_{1}^{\hat{t}} b_{1}\right): F_{2} & \longrightarrow F_{2} \\
a_{2} & \longmapsto a_{2}^{-1} \\
b_{2} & \longmapsto a_{2}^{2(\hat{t})+1} b_{2} a_{2} .
\end{aligned}
$$

We can naturally extend this definition for even integers $t$, by

$$
\begin{aligned}
\mu_{t}: F_{2} & \longrightarrow F_{2} \\
a_{2} & \longmapsto a_{2}^{-1} \\
b_{2} & \longmapsto a_{2}^{t} b_{2} a_{2} .
\end{aligned}
$$

Since it is no extra effort we solve equation (4.13) for all non-zero integers $t \in \mathbb{Z}$.

One example of solution for this equation is the word $C_{0}=a_{2}$ and consequently the whole subgroup $\left\langle C_{0}\right\rangle$. Another, not so obvious one is the word $C_{0}^{t} C_{1}=a_{2}^{t} b_{2} a_{2} b_{2}^{-1}$. Indeed

$$
\begin{aligned}
\mu_{t}\left(C_{0}^{t} C_{1}\right) & =C_{0}^{-t}\left(C_{0}^{-1} \zeta_{1}(t+1) C_{1}^{-1} \zeta_{1}^{-1}(t+1) C_{0}\right) \\
& =C_{0}^{-t}\left(C_{0}^{-1} C_{0}^{t+1} C_{1}^{-1} C_{0}^{-t-1} C_{0}\right) \\
& =C_{1}^{-1} C_{0}^{-t} \\
& =\left(C_{0}^{t} C_{1}\right)^{-1} .
\end{aligned}
$$

It is possible to generalize these kind of solutions using the following Proposition.

Proposition 4.2.10. For all $n, s, t \in \mathbb{Z}$ with $t \neq 0$, we have that

$$
\mu_{t}\left(C_{0}^{-1} \zeta_{n}(t+1) C_{n}^{s}\right)=\left(C_{0}^{-1} \zeta_{n}(t+1) C_{n}^{s}\right)^{-1} .
$$

Proof. From Proposition 4.2.6 we know that

$$
\mu_{t}\left(C_{n}^{s}\right)=C_{0}^{-1} \zeta_{n}(t+1)\left(C_{n}\right)^{-s} \zeta_{n}^{-1}(t+1) C_{0} .
$$

Also, from Corollary 4.2.9.1 it holds for all $n \in \mathbb{Z}$

$$
\mu_{t}\left(\zeta_{n}(t+1)\right)=C_{0}^{-1} \zeta_{n}^{-1}(t+1) C_{0} .
$$

Therefore

$$
\begin{aligned}
\mu_{t}\left(C_{0}^{-1} \zeta_{n}(t+1) C_{n}^{s}\right) & =\mu_{t}\left(C_{0}\right)^{-1} \mu_{t}\left(\zeta_{n}(t+1)\right) \mu_{t}\left(C_{1}\right)^{s} \\
& =C_{0} C_{0}^{-1} \zeta_{n}^{-1}(t+1) C_{0} C_{0}^{-1} \zeta_{n}(t+1)\left(C_{n}\right)^{-s} \zeta_{n}^{-1}(t+1) C_{0} \\
& =\left(C_{n}\right)^{-s} \zeta_{n}^{-1}(t+1) C_{0} \\
& =\left(C_{0}^{-1} \zeta_{n}(t+1) C_{n}^{s}\right)^{-1}
\end{aligned}
$$


To simplify notation set for every $n \geq 0, s, t \in \mathbb{Z}$

$$
\xi_{n}^{s}(t+1)=C_{0}^{-1} \zeta_{n}(t+1) C_{n}^{s}=C_{0}^{t} C_{1}^{t+1} \cdot \ldots \cdot C_{n-1}^{t+1} C_{n}^{s} .
$$

Note that $\xi_{n}^{s}(t+1)$ is a solution of equation (4.13), where $t \in \mathbb{Z}$ is odd.

Corollary 4.2.10.1. For every $n \geq 0$ and $s \in \mathbb{Z}$ it holds that

$$
\mu_{t}\left(\xi_{n}^{s}(t+1)\right)=\left(\xi_{n}^{s}(t+1)\right)^{-1} .
$$

A twisted conjugacy of a solution of equation (4.13) is also a solution.

Observation 4.2.11. Consider the word $w=u v \mu_{t}\left(u^{-1}\right)$, where $u, v \in F_{2}$ and $\mu_{t}(v)=v^{-1}$. If we apply $\mu_{t}$ on $w$ we get

$$
\mu_{t}(w)=\mu_{t}\left(u v \mu_{t}\left(u^{-1}\right)\right)=\mu_{t}(u) \mu_{t}(v) \mu_{t}^{2}\left(u^{-1}\right)=\mu_{t}(u) v^{-1} u^{-1}=w^{-1} .
$$

Therefore words that can be written in this form are solutions to the equation (4.13).

Indeed it is possible to show that every solution can be written in this form, where $v=\xi_{n}^{s}(t+1)$ for some $s, t, n \in \mathbb{Z}$. The idea behind that proof is to write the word $w$ as product of words in subsets of $H$. For example, if

$$
w=u_{1} v_{1} \cdot \ldots \cdot u_{l} v_{l}
$$

with $u_{i} \in G_{1}$ and $v_{j} \in G_{2}$, where $G_{1}$ and $G_{2}$ are subsets of $H$ with $G_{1} \cap G_{2}=\{1\}$. Then

$$
\mu_{t}\left(w^{-1}\right)=\mu_{t}\left(v_{l}^{-1}\right) \mu_{t}\left(u_{l}^{-1}\right) \cdot \ldots \cdot \mu_{t}\left(v_{1}^{-1}\right) \mu_{t}\left(u_{1}^{-1}\right) .
$$

If we choose $G_{1}$ and $G_{2}$ in a way that they are invariant in respect to $\mu_{t}$, i.e. $\mu_{t}\left(G_{1}\right) \subset G_{1}$ and $\mu_{t}\left(G_{2}\right) \subset G_{2}$ then it is possible to compare the words and get more information about $u_{i}$ and $v_{j}$ and consequently about $w$.

One invariant subset of $H$ is $H_{n}=\left\langle C_{i}: 0 \leq|i| \leq|n|\right\rangle$. The invariancy follows from Corollary 4.2.6 and the fact that $\zeta_{n}(t+1) \in H_{n}$. Note, that for a fixed non-zero $n$ the subsets $H_{n-\operatorname{sgn}(n)}$ and $\left\langle C_{n}\right\rangle$ intersect only in the empty word. And even though $\left\langle C_{n}\right\rangle$ is not a $\mu_{t}$ invariant subgroup it is possible to use that idea to find the solutions in the subgroups $H^{+}:=\left\langle C_{n}: n \geq 0\right\rangle=\bigcup_{n \geq 0} H_{n}$ and $H^{-}:=\left\langle C_{n}: n \leq 0\right\rangle=\bigcup_{n \leq 0} H_{n}$.

Theorem 4.2.12. Let $t$ be a non-zero integer and let $w$ be a word either in $H^{+}=\left\langle C_{n}: 0 \leq n\right\rangle$ or in $H^{-}=\left\langle C_{n}: 0 \geq n\right\rangle$ with $\mu_{t}(w)=w^{-1}$, then there exist integers $n, s \in \mathbb{Z}$ with $n \neq 0$, as well as a word $u \in H$ such that

$$
w=u \xi_{n}^{s}(t+1) \mu_{t}\left(u^{-1}\right)
$$

Proof. We will show using induction to $n$ that if $w \in H_{n}$ with $\mu_{t}(w)=w^{-1}$, then there are integers $s, \hat{n} \in Z$, with $0 \leq|\hat{n}| \leq|n|$ and there is a word $u \in H$ such that

$$
w=u \xi_{\hat{n}}^{s}(t+1) \mu_{t}\left(u^{-1}\right)
$$

For the induction, let $n=0$. Then a word $w=C_{0}^{s}$ in $H_{0}$ satisfies $\mu_{t}(w)=w^{-1}$ and therefore is a solution to the equation and can be written as $u v \mu_{t}\left(u^{-1}\right)$ in the desired form, setting $u=1$ and $v=w=\xi_{0}^{s}(t+1)$. 
Suppose that the hypothesis holds for $n-\operatorname{sing}(n)$, for $n \neq 0$, let's show it for $n$. Let $w \in$ $H_{n} \backslash H_{n-\operatorname{sgn}(n)}$ with $\mu_{t}\left(w^{-1}\right)=w$. We can write $w$ in the following form

$$
w=u_{1} C_{n}^{s_{1}} u_{2} C_{n}^{s_{2}} \cdot \ldots \cdot u_{l} C_{n}^{s_{l}},
$$

where $u_{i} \in H_{n-\operatorname{sgn}(n)}$ and $s_{i} \in \mathbb{Z}$ and $u_{2}, \ldots u_{l}$ non-empty words and $s_{1}, \ldots, s_{l-1}$ not zero. Applying $\mu_{t}$ on $w^{-1}$ we obtain

$$
\begin{aligned}
\mu_{t}\left(w^{-1}\right) & =\mu_{t}\left(C_{n}^{-s_{l}} u_{l}^{-1} C_{n}^{-s_{l-1}} u_{l-1}^{-1} \cdot \ldots \cdot C_{n}^{-s_{1}} u_{1}^{-1}\right) \\
& =\left[\left(C_{0}^{-1} \zeta_{n}(t+1)\right) C_{n}^{s_{l}}\left(\zeta_{n}^{-1}(t+1) C_{0}\right)\right] \mu_{t}\left(u_{l}\right)^{-1} \ldots\left[\left(C_{0}^{-1} \zeta_{n}(t+1)\right) C_{n}^{s_{1}}\left(\zeta_{n}^{-1}(t+1) C_{0} \mu_{t}\left(u_{1}^{-1}\right)\right)\right. \\
& \left.=\left[C_{0}^{-1} \zeta_{n}(t+1)\right] C_{n}^{s_{l}}\left[\left(\zeta_{n}^{-1}(t+1) C_{0}\right) \mu_{t}\left(u_{l}\right)^{-1}\left(C_{0}^{-1} \zeta_{n}(t+1)\right)\right] \ldots\left[\left(\zeta_{n}^{-1}(t+1) C_{0}\right) \mu_{t}\left(u_{1}^{-1}\right)\right)\right] .
\end{aligned}
$$

Since $\left(C_{0}^{-1} \zeta_{n}(t+1)\right) \in H_{n-\operatorname{sgn}(n)}$ and $\mu_{t}\left(w^{-1}\right)=w$ we can compare the parts of these two words. We don't know if $s_{l}$ is zero or not, neither if $u_{1}$ is the empty word or not. So we divide in cases and see what happens in each case.

a) Suppose that $s_{l} \neq 0$, then

$$
u_{1}=C_{0}^{-1} \zeta_{n}(t+1)
$$

which, since $\mu_{t}\left(\zeta_{n}(t+1)\right)=C_{0}^{-1} \zeta_{n}^{-1}(t+1) C_{0}$, is equivalent to

$$
\mu_{t}\left(u_{1}^{-1}\right)=C_{0}^{-1} \zeta_{n}(t+1) .
$$

Hence $u_{1}$ is only zero, if $n=1$ and $t=0$, but this is not possible by hypothesis. Also it holds for every $i, j$ such that $2 \leq i \leq l$ and $1 \leq j \leq l$ that

$$
\begin{aligned}
& u_{i}=\left(\zeta_{n}^{-1}(t+1) C_{0}\right) \mu_{t}\left(u_{l-i+2}\right)^{-1}\left(C_{0}^{-1} \zeta_{n}(t+1)\right) \\
& s_{j}=s_{l-j+1} .
\end{aligned}
$$

- Suppose that $l=2 \hat{l}$ is even, then

$$
\begin{aligned}
\left(\zeta_{n}^{-1}(t+1) C_{0}\right) \mu\left(u_{\hat{l}-i+1}^{-1}\left(C_{0}^{-1} \zeta_{n}(t+1)\right)\right. & =\left(\zeta_{n}^{-1}(t+1) C_{0}\right) \mu\left(u_{l-(\hat{l}+i+1)+2}^{-1}\right)\left(C_{0}^{-1} \zeta_{n}(t+1)\right) \\
& =u_{\hat{l}+i+1}
\end{aligned}
$$

$0 \leq i \leq \hat{l}-1$ and

$$
s_{\hat{l}-j}=s_{l-(\hat{l}+j+1)+1}=s_{\hat{l}+j+1}
$$

for $0 \leq j \leq \hat{l}-1$. Note that if we set

$$
\hat{v}=u_{\hat{l}+1}\left(\zeta_{n}^{-1}(t+1) C_{0}\right)
$$

we obtain

$$
\begin{aligned}
\mu_{t}\left(\hat{v}^{-1}\right) & =\mu_{t}\left(\left(u_{\hat{l}+1}\left(\zeta_{n}^{-1}(t+1) C_{0}\right)\right)^{-1}\right. \\
& \left.=\mu_{t}\left(\zeta_{n}^{-1}(t+1) C_{0}\right)^{-1}\right) \mu_{t}\left(u_{\hat{l}+1}^{-1}\right) \\
& \left.=\mu_{t}\left(\zeta_{n}^{-1}(t+1) C_{0}\right)^{-1}\right) \mu_{t}\left(u_{\hat{l}+1}^{-1}\right) \\
& =\left(\zeta_{n}^{-1}(t+1) C_{0}\right) \mu_{t}\left(u_{\hat{l}+1}^{-1}\right) \\
& =u_{\hat{l}+i+1}\left(\zeta_{n}^{-1}(t+1) C_{0}\right) \\
& =\hat{v}
\end{aligned}
$$


Since this is a word in $H_{n-\operatorname{sgn}(n)}$ it follows by induction hypothesis that there are integers $s, \hat{n} \in Z$, with $0 \leq|\hat{n}| \leq|n-\operatorname{sgn}(n)|<|n|$ and a word $\hat{u} \in H$ such that

$$
\hat{v}=\hat{u} \xi_{\hat{n}}^{s}(t+1) \mu_{t}\left(\hat{u}^{-1}\right),
$$

Set

$$
u=u_{1} C_{n}^{s_{1}} \cdot \ldots \cdot u_{\hat{l}} C_{n}^{s_{\hat{l}}} \hat{u}
$$

It follows that

$$
\begin{aligned}
& \mu_{t}\left(u^{-1}\right)=\mu_{t}\left(\hat{u}^{-1} C_{n}^{-s_{\hat{l}}} u_{\hat{l}}^{-1} C_{n}^{-s_{\hat{l}-1}} u_{\hat{l}-1}^{-1} \cdot \ldots \cdot C_{n}^{-s_{1}} u_{1}^{-1}\right) \\
& \quad=\mu_{t}\left(\hat{u}^{-1}\right)\left[\left(C_{0}^{-1} \zeta_{n}(t+1)\right) C_{n}^{s_{\hat{l}}}\left(\zeta_{n}^{-1}(t+1) C_{0}\right)\right] \mu\left(u_{\hat{l}}^{-1}\right) \ldots\left[\left(C_{0}^{-1} \zeta_{n}(t+1)\right) C_{n}^{s_{1}}\left(\zeta_{n}^{-1}(t+1) C_{0}\right)\right] \mu\left(u_{1}^{-1}\right) \\
& \quad=\mu_{t}\left(\hat{u}^{-1}\right)\left(C_{0}^{-1} \zeta_{n}(t+1)\right) C_{n}^{s_{\hat{l}}}\left[\left(\zeta_{n}^{-1}(t+1) C_{0}\right) \mu\left(u_{\hat{l}}^{-1}\right)\left(C_{0}^{-1} \zeta_{n}(t+1)\right)\right] \ldots C_{n}^{s_{1}}\left[\zeta_{n}^{-1}(t+1) C_{0} \mu\left(u_{1}^{-1}\right)\right] \\
& \quad=\left[\mu_{t}\left(\hat{u}^{-1}\right)\left(C_{0}^{-1} \zeta_{n}(t+1)\right)\right] C_{n}^{s_{\hat{l}+1}} u_{\hat{l}+2} C_{n}^{s_{\hat{l}+2}} \cdot \ldots \cdot u_{l} C_{n}^{s_{l}}
\end{aligned}
$$

Therefore

$$
\begin{aligned}
u \xi_{\hat{n}}^{s}(t+1) \mu_{t}\left(u^{-1}\right) & =\left[u_{1} C_{n}^{s_{1}} \ldots u_{\hat{l}} C_{n}^{s_{\hat{\imath}}} \hat{u}\right] \xi_{\hat{n}}^{s}(t+1)\left[\mu_{t}\left(\hat{u}^{-1}\right)\left(C_{0}^{-1} \zeta_{n}(t+1)\right) C_{n}^{s_{\hat{l}+1}} u_{\hat{l}+2} \ldots u_{l} C_{n}^{s_{l}}\right] \\
& =\left[u_{1} C_{n}^{s_{1}} \ldots u_{\hat{l}} C_{n}^{s_{\hat{\imath}}}\right]\left[u_{\hat{l}+1}\left(\zeta_{n}^{-1}(t+1) C_{0}\right)\right]\left[\left(C_{0}^{-1} \zeta_{n}(t+1)\right) C_{n}^{s_{\hat{l}+1}} u_{\hat{l}+2} \ldots u_{l} C_{n}^{s_{l}}\right] \\
& =u_{1} C_{n}^{s_{1}} \ldots \cdot u_{l} C_{n}^{s_{l}} \\
& =w .
\end{aligned}
$$

Hence, we managed to write $w$ in the form we wanted.

- If $l=2 \hat{l}+1$ is odd, then set

$$
\begin{aligned}
& u=u_{1} C_{n}^{s_{1}} \cdot \ldots \cdot C_{n}^{s_{\hat{\imath}}} u_{\hat{l}+1}\left(\zeta_{n}^{-1}(t+1) C_{0}\right) \\
& v=\left(C_{0}^{-1} \zeta_{n}(t+1)\right) C_{n}^{s_{\hat{l}+1}}=\xi_{n}^{s_{\hat{l}+1}}(t+1)
\end{aligned}
$$

Using that $l-\hat{l}=\hat{l}+1$ we get

$$
\begin{aligned}
\left(\zeta_{n}^{-1}(t+1) C_{0}\right) \mu\left(u_{(\hat{l}+1)-i}^{-1}\right)\left(C_{0}^{-1} \zeta_{n}(t+1)\right) & =\left(\zeta_{n}^{-1}(t+1) C_{0}\right) \mu\left(u_{l-(\hat{l}+i+2)+2}^{-1}\right)\left(C_{0}^{-1} \zeta_{n}(t+1)\right) \\
& =u_{\hat{l}+i+2}
\end{aligned}
$$

for $0 \leq i \leq \hat{l}-1$ and

$$
s_{\hat{l}-j}=s_{l-(\hat{l}+j+2)+1}=s_{\hat{l}+j+2}
$$

for $0 \leq j \leq \hat{l}-1$ Computing $\mu_{t}$ on $u^{-1}$ implies

$$
\begin{aligned}
\mu_{t}\left(u^{-1}\right) & =\mu_{t}\left(\left(C_{0}^{-1} \zeta_{n}(t+1)\right) u_{\hat{l}+1}^{-1} C_{n}^{-s_{\hat{\imath}}} \cdot \ldots \cdot C_{n}^{-s_{1}} u_{1}^{-1}\right) \\
& =\left[\left(\zeta_{n}^{-1}(t+1) C_{0}\right) \mu_{t}\left(u_{\hat{l}+1}^{-1} C_{0}^{-1} \zeta_{n}(t+1)\right] C_{n}^{s_{\hat{l}}} \ldots C_{n}^{s_{1}}\left[\zeta_{n}^{-1}(t+1) C_{0} \mu\left(u_{1}^{-1}\right)\right]\right. \\
& =u_{\hat{l}+2} C_{n}^{s_{\hat{l}+2}} \cdot \ldots \cdot u_{l} C_{n}^{s_{l}} .
\end{aligned}
$$


Therefore

$$
\begin{aligned}
u v \mu_{t}\left(u^{-1}\right) & =\left[u_{1} C_{n}^{s_{1}} \ldots C_{n}^{s_{\hat{l}}} u_{\hat{l}+1}\left(\zeta_{n}^{-1}(t+1) C_{0}\right)\right]\left[\left(C_{0}^{-1} \zeta_{n}(t+1) C_{n}^{s_{\hat{l}+1}}\right] u_{\hat{l}+2} C_{n}^{s_{\hat{l}+2}}\right. \\
& \left.\ldots u_{l} C_{n}^{s_{l}} u_{l+1}\left(\zeta_{n}^{-1}(t+1) C_{0}\right)\right) \\
& =u_{1} C_{n}^{s_{1}} \cdot \ldots \cdot u_{l} C_{n}^{s_{l}} \\
& =w .
\end{aligned}
$$

Hence $w=u \xi_{n}^{s_{\hat{l}+1}}(t+1) \mu_{t}\left(u^{-1}\right)$.

b) Suppose that $s_{l}=0$ and $u_{1}=1$, then

$$
\begin{aligned}
w & =C_{n}^{s_{1}} u_{2} \cdot \ldots \cdot C_{n}^{s_{l-1}} u_{l}, \\
\mu_{t}\left(w^{-1}\right) & \left.=\left[\mu_{t}\left(u_{l}\right)^{-1}\left(C_{0}^{-1} \zeta_{n}(t+1)\right)\right] C_{n}^{s_{l-1}} \ldots \mu_{t}\left(u_{2}\right)^{-1} C_{0}^{-1} \zeta_{n}(t+1)\right] C_{n}^{s_{1}}\left[\zeta_{n}^{-1}(t+1) C_{0}\right] .
\end{aligned}
$$

Set $\hat{w}=w^{-1}$, then $\mu_{t}\left(\hat{w}^{-1}\right)=\mu_{t}(w)$ and therefore $\mu_{t}\left(\hat{w}^{-1}\right)=\hat{w}$. Thus $\hat{w}$ fits in the previous case, hence there are words $\hat{u}, \hat{v}$ such that

$$
\hat{w}=\hat{u} \hat{v} \mu_{t}\left(\hat{u}^{-1}\right),
$$

with $\hat{u} \in H$ and $\hat{v}$ either in $H_{n-\operatorname{sgn}(n)}$ or $\hat{v}=\xi_{n}^{s}(t+1)$ for some $s \in \mathbb{Z}$.

If $\hat{v} \in H_{n-\operatorname{sgn}(n)}$, set $u=\mu_{t}(\hat{u})$ and $v=\hat{v}^{-1}$. Then $\mu_{t}(u)=\hat{u}$ and

$$
w=\hat{w}^{-1}=\mu_{t}(\hat{u}) \hat{v}^{-1} \hat{u}^{-1}=u v \mu_{t}\left(u^{-1}\right) .
$$

If $\hat{v}=\xi_{n}^{s}=\left(C_{0}^{-1} \zeta_{n}(t+1)\right) C_{n}^{s}$ for some $s \in \mathbb{Z}$ then set

$$
\begin{aligned}
& u=\mu_{t}(\hat{u})\left(\zeta_{n}(t+1)^{-1} C_{0}\right), \\
& v=\left(C_{0}^{-1} \zeta_{n}(t+1)\right) C_{n}^{-s}=\xi_{n}^{-s}(t+1) .
\end{aligned}
$$

Then

$$
\mu_{t}\left(u^{-1}\right)=\mu_{t}\left(\left(\zeta_{n}(t+1)^{-1} C_{0}\right)^{-1} \mu_{t}\left(\hat{u}^{-1}\right)\right)=\left(\zeta_{n}(t+1)^{-1} C_{0}\right) \hat{u}^{-1}
$$

and

$$
\begin{aligned}
w & =\hat{w}^{-1}=\mu_{t}(\hat{u}) \hat{v}^{-1} \hat{u}^{-1} \\
& =\mu_{t}(\hat{u}) C_{n}^{-s}\left(\zeta_{n}(t+1)^{-1} C_{0}\right) \hat{u}^{-1} \\
& =\left[\mu_{t}(\hat{u})\left(\zeta_{n}(t+1)^{-1} C_{0}\right)\right]\left[\left(C_{0}^{-1} \zeta_{n}(t+1) C_{n}^{-s}\right] \mu_{t}\left(u^{-1}\right)\right. \\
& =u v \mu_{t}\left(u^{-1}\right) \\
& =u \xi_{n}^{-s}(t+1) \mu_{t}\left(u^{-1}\right)
\end{aligned}
$$

c) Suppose that $s_{l}=0$ and $u_{1} \neq 1$, then from $w_{1}=\mu_{t}\left(w_{1}\right)^{-1}$ it follows

$$
\begin{aligned}
& u_{1} C_{n}^{s_{1}} u_{2} C_{n}^{s_{2}} \cdot \ldots \cdot C_{n}^{s_{l-1}} u_{l}, \\
& \left.\quad=\left[\mu_{t}\left(u_{l}\right)^{-1}\left(C_{0}^{-1} \zeta_{n}(t+1)\right)\right] C_{n}^{s_{l-1}} \cdot \ldots \cdot C_{0}^{-1} \zeta_{n}(t+1)\right] C_{n}^{s_{1}}\left[\left(\zeta_{n}^{-1}(t+1) C_{0}\right) \mu_{t}\left(u_{1}^{-1}\right)\right] .
\end{aligned}
$$

Therefore

$$
u_{1}=\mu_{t}\left(u_{l}\right)^{-1}\left(C_{0}^{-1} \zeta_{n}(t+1)\right),
$$


which is equivalent to

$$
u_{l}=\left(\zeta_{n}^{-1}(t+1) C_{0}\right) \mu_{t}\left(u_{1}^{-1}\right)
$$

Also

$$
\begin{aligned}
& u_{i}=\left(\zeta_{n}^{-1}(t+1) C_{0}\right) \mu_{t}\left(u_{l-i+1}\right)^{-1}\left(C_{0}^{-1} \zeta_{n}(t+1)\right) \\
& s_{j}=s_{l-j},
\end{aligned}
$$

for every $i, j$ such that $2 \leq i \leq l-1$ and $1 \leq j \leq l-1$.

- Suppose that $l=2 \hat{l}$ is even, then set

$$
\begin{aligned}
& u=u_{1} C_{n}^{s_{1}} \cdot \ldots \cdot C_{n}^{s_{\hat{l}-1}} u_{\hat{l}}\left(\zeta_{n}^{-1}(t+1) C_{0}\right) \\
& v=\left(\left(C_{0}^{-1} \zeta_{n}(t+1)\right) C_{n}^{s_{\hat{\imath}}}=\xi_{n}^{s_{\hat{l}}}(t+1)\right.
\end{aligned}
$$

Using that $l-\hat{l}=\hat{l}$ we get, for $0 \leq i \leq \hat{l}-1$ that

$$
\begin{aligned}
\left(\zeta_{n}^{-1}(t+1) C_{0}\right) \mu\left(u_{\hat{l}-i}^{-1}\right)\left(C_{0}^{-1} \zeta_{n}(t+1)\right) & =\left(\zeta_{n}^{-1}(t+1) C_{0}\right) \mu\left(u_{l-(\hat{l}+i+1)+1}^{-1}\right)\left(C_{0}^{-1} \zeta_{n}(t+1)\right) \\
& =u_{\hat{l}+i+1}
\end{aligned}
$$

and for $0 \leq j \leq \hat{l}-1$

$$
s_{\hat{l}-j}=s_{l-(\hat{l}+j)}=s_{\hat{l}+j} .
$$

Applying $\mu_{t}$ on $u^{-1}$ implies

$$
\begin{aligned}
\mu_{t}\left(u^{-1}\right) & =\mu_{t}\left(\left(\left(C_{0}^{-1} \zeta_{n}(t+1)\right) u_{\hat{l}}^{-1} C_{n}^{-s_{\hat{l}-1}} \ldots C_{n}^{-s_{1}} u_{1}^{-1}\right)\right. \\
& =\left[\left(\zeta_{n}^{-1}(t+1) C_{0}\right) \mu_{t}\left(u_{\hat{l}}^{-1} C_{0}^{-1} \zeta_{n}(t+1)\right] C_{n}^{s_{\hat{l}-1}} \ldots C_{n}^{s_{1}}\left[\zeta_{n}^{-1}(t+1) C_{0} \mu\left(u_{1}^{-1}\right)\right]\right. \\
& =u_{\hat{l}+1} C_{n}^{s_{\hat{l}+1}} \ldots \cdot u_{l-1} C_{n}^{s_{l-1}} u_{l} .
\end{aligned}
$$

Therefore

$$
\begin{aligned}
u v \mu_{t}\left(u^{-1}\right) & =\left[u_{1} C_{n}^{s_{1}} \ldots C_{n}^{s_{\hat{l}-1}} u_{\hat{l}}\left(\zeta_{n}^{-1}(t+1) C_{0}\right)\right]\left[\left(C_{0}^{-1} \zeta_{n}(t+1)\right) C_{n}^{s_{\hat{t}}}\right]\left[u_{\hat{l}+1} C_{n}^{s_{\hat{l}+1}} \ldots u_{l-1} C_{n}^{s_{l-1}} u_{l}\right] \\
& =u_{1} C_{n}^{s_{1}} \cdot \ldots C_{n}^{s_{l-1}} u_{l} \\
& =w .
\end{aligned}
$$

- If $l=2 \hat{l}+1$ is odd, then

$$
\begin{aligned}
\left(\zeta_{n}^{-1}(t+1) C_{0}\right) \mu\left(u_{\hat{l}-i+1}^{-1}\right)\left(C_{0}^{-1} \zeta_{n}(t+1)\right) & =\left(\zeta_{n}^{-1}(t+1) C_{0}\right) \mu\left(u_{l-(\hat{l}+i+1)+1}^{-1}\right)\left(C_{0}^{-1} \zeta_{n}(t+1)\right) \\
& =u_{\hat{l}+i+1}
\end{aligned}
$$

$0 \leq i \leq \hat{l}-1$ and

$$
s_{\hat{l}-j}=s_{l-(\hat{l}+j+1)}=s_{\hat{l}+j+1}
$$

for $0 \leq j \leq \hat{l}-1$. Set

$$
\hat{v}=u_{\hat{l}+1}\left(\zeta_{n}^{-1}(t+1) C_{0}\right),
$$


then

$$
\begin{aligned}
\mu_{t}(\hat{v}) & =\mu_{t}\left(u_{\hat{l}+1}\right) \mu_{t}\left(\zeta_{n}^{-1}(t+1)\right) \mu_{t}\left(C_{0}\right) \\
& \left.=\left(C_{0}^{-1} \zeta_{n}(t+1)\right) u_{\hat{l}+1}^{-1} \zeta_{n}^{-1}(t+1) C_{0}\right)\left(C_{0}^{-1} \zeta_{n}(t+1) C_{0}\right) C_{0}^{-1} \\
& =\left(u_{\hat{l}+1} \zeta_{n}^{-1}(t+1) C_{0}\right)^{-1} \\
& =\hat{v}^{-1}
\end{aligned}
$$

Considering that $\hat{v} \in H_{n-\operatorname{sgn}(n)}$ this implies, by hypothesis, that there are integers $s, \hat{n} \in Z$, with $0 \leq|\hat{n}| \leq|n-\operatorname{sgn}(n)|<|n|$ and a word $\hat{u} \in H$ such that

$$
\hat{v}=\hat{u} \xi_{\hat{n}}^{s}(t+1) \mu_{t}\left(\hat{u}^{-1}\right) \text {. }
$$

Set

$$
u=u_{1} C_{n}^{s_{1}} \cdot \ldots \cdot u_{\hat{l}} C_{n}^{s_{\hat{l}}} \hat{u} .
$$

Applying $\mu_{t}$ on $u$ gives us

$$
\begin{aligned}
& \mu_{t}\left(u^{-1}\right)=\mu\left(\hat{u}^{-1} C_{n}^{-s_{\hat{l}}} u_{\hat{l}}^{-1} C_{n}^{-s_{\hat{l}-1}} u_{\hat{l}-1}^{-1} \cdot \ldots \cdot C_{n}^{-s_{1}} u_{1}^{-1}\right) \\
& \quad=\mu\left(\hat{u}^{-1}\right)\left(C_{0}^{-1} \zeta_{n}(t+1)\right) C_{n}^{s_{\hat{\imath}}}\left[\left(\zeta_{n}^{-1}(t+1) C_{0}\right) \mu\left(u_{\hat{l}}^{-1}\right) \ldots C_{n}^{s_{1}}\left[\left(\zeta_{n}^{-1}(t+1) C_{0}\right) \mu\left(u_{1}^{-1}\right)\right]\right. \\
& \quad=\mu\left(\hat{u}^{-1}\right)\left(C_{0}^{-1} \zeta_{n}(t+1)\right) C_{n}^{s_{\hat{l}+1}} u_{\hat{l}+2} C_{n}^{s_{\hat{l}+2}} \cdot \ldots \cdot C_{n}^{s_{l-1}} u_{l} .
\end{aligned}
$$

Therefore

$$
\begin{aligned}
& u \xi_{\hat{n}}^{s}(t+1) \mu_{t}\left(u^{-1}\right) \\
& \quad=\left[u_{1} C_{n}^{s_{1}} \ldots u_{\hat{l}} C_{n}^{s_{\hat{l}} \hat{u}}\right] \xi_{\hat{n}}^{s}(t+1)\left[\mu_{t}\left(\hat{u}^{-1}\right)\left(C_{0}^{-1} \zeta_{n}(t+1)\right) C_{n}^{s_{\hat{l}+1}} u_{\hat{l}+2} \ldots C_{n}^{s_{l-1}} u_{l}\right] \\
& \quad=\left[u_{1} C_{n}^{s_{1}} \ldots u_{\hat{l}} C_{n}^{s_{\hat{l}}}\left[\hat{u} \xi_{\hat{n}}^{s^{s}}(t+1) \mu_{t}\left(\hat{u}^{-1}\right)\right]\left(C_{0}^{-1} \zeta_{n}(t+1)\right) C_{n}^{s_{l+1}} u_{\hat{l}+2} \ldots C_{n}^{s_{l-1}} u_{l}\right] \\
& \quad=\left[u_{1} C_{n}^{s_{1}} \ldots u_{\hat{l}} C_{n}^{s_{\hat{l}}}\right] \hat{v}\left[\left(C_{0}^{-1} \zeta_{n}(t+1)\right) C_{n}^{s_{\hat{l}+1}} u_{\hat{l}+2} \ldots C_{n}^{s_{l-1}} u_{l}\right] \\
& =\left[u_{1} C_{n}^{s_{1}} \ldots u_{\hat{l}} C_{n}^{s_{\hat{\imath}}}\right]\left[u_{\hat{l}+1}\left(\zeta_{n}^{-1}(t+1) C_{0}\right)\right]\left[\left(C_{0}^{-1} \zeta_{n}(t+1)\right) C_{n}^{s_{\hat{l}+1}} u_{\hat{l}+2} \ldots C_{n}^{s_{l-1}} u_{l}\right] \\
& =u_{1} C_{n}^{s_{1}} \ldots C_{n}^{s_{l-1}} u_{l} \\
& \quad=w .
\end{aligned}
$$

Consider the following two subgroups of $H$

$$
\begin{aligned}
H^{+} & =\left\langle C_{i}: 0 \leq i\right\rangle \\
G^{-} & =H^{-} \backslash\left\langle C_{0}\right\rangle=\left\langle C_{i}: 0>i\right\rangle \subseteq H^{-} .
\end{aligned}
$$

These are subgroups of $H$, whose free product, $H^{+} * G^{-}$, is $H$. Therefore every word in $H$ is a finite product of words in $H^{+}$and words in $G^{-}$. Then, in a similar way as in the last Theorem, it is possible to solve equation 4.13 .

Theorem 4.2.13. Let $w \in H=\left\langle C_{i}: i \in \mathbb{Z}\right\rangle$ with $\mu_{t}(w)=w^{-1}$, then there exists the word $u$ in $H$ and $s, n \in \mathbb{Z}$ such that

$$
w=u \xi_{n}^{s}(t+1) \mu_{t}\left(u^{-1}\right) .
$$

Conversely, if $w=u \xi_{n}^{s} \theta(u)$ with $u \in H$ and $n, s \in \mathbb{Z}$, then $\mu(w)=w^{-1}$. 
Proof. Since $H=H^{+} * G^{-}$it is possible to write, in a unique way,

$$
w=u_{1} v_{1} \cdot \ldots \cdot u_{l} v_{l},
$$

where $u_{i} \in H^{+}$and $v_{i} \in G^{-}$, for all $i \in\{1, \ldots l\}$ and $u_{2}, \ldots, u_{l} \neq 1$, as well as $v_{1}, \ldots, v_{l-1} \neq 1$. Applying $\mu_{t}$ on $w$ we obtain

$$
\begin{aligned}
\mu_{t}\left(w^{-1}\right) & =\mu_{t}\left(v_{l}^{-1}\right) \mu_{t}\left(u_{l}^{-1}\right) \cdot \ldots \cdot \mu_{t}\left(v_{1}^{-1}\right) \mu_{t}\left(u_{1}^{-1}\right) \\
& =C_{0}^{-1}\left[C_{0} \mu_{t}\left(v_{l}^{-1}\right) C_{0}^{-1}\right]\left[C_{0} \mu_{t}\left(u_{l}^{-1}\right) C_{0}^{-1}\right] \ldots\left[C_{0} \mu_{t}\left(v_{1}^{-1}\right) C_{0}^{-1}\right]\left[C_{0} \mu_{t}\left(u_{1}^{-1}\right)\right] .
\end{aligned}
$$

It holds that $\mu_{t}\left(H^{+}\right) \subseteq H^{+}$, also $C_{0} \in H^{+}$. By Proposition 4.2 .6

$$
\mu_{t}\left(C_{n}\right)=C_{0}^{-1} \zeta_{n}(t+1) C_{n}^{-1} \zeta_{n}^{-1}(t+1) C_{0}
$$

Since $\zeta_{-1}=1$ and for $n<-1$ it holds that

$$
\zeta_{n}(t)=C_{-1}^{-t} \cdot \ldots \cdot C_{n+1}^{-t},
$$

(see (4.18)), it follows that $\zeta_{n}(t+1) \in G^{-}$. Hence

$$
C_{0} \mu_{t}\left(C_{n}\right) C_{0}^{-1}=\zeta_{n}(t+1) C_{n}^{-1} \zeta_{n}^{-1}(t+1)
$$

is in $G^{-}$for every $n \leq-1$ and consequently $C_{0} \mu_{t}\left(v_{l}^{-1}\right) C_{0}^{-1} \in G^{-}$. Then both, $w$ and $\mu_{t}\left(w^{-1}\right)$, have been written as a product of words in $H^{+}$and $G^{-}$. Since $H=H^{+} * G^{-}$and $w=\mu_{t}\left(w^{-1}\right)$ this product is unique, thus the words are equal. Similar to the proof of Theorem 4.2.12 we divide into the cases:
a) $u_{1}, v_{l} \neq 1$,
b) $u_{1}, v_{l}=1$,
c) $u_{1} \neq 1$ and $v_{l}=1$,
d) $u_{1}=1$ and $v_{l} \neq 1$.

All these cases are solved with the same technique and the proof is very similar to the proof of Theorem 4.2 .12 , that is why we will only write the first case.

a) Assume that $u_{1}, v_{l} \neq 1$ and let $l=2 \hat{l}$ be even. Then

$$
\begin{aligned}
& u_{1}=C_{0}^{-1}, \\
& u_{i}=C_{0} \mu_{t}\left(u_{l-i+2}^{-1}\right) C_{0}^{-1}, \\
& v_{j}=C_{0} \mu_{t}\left(v_{l-j+1}^{-1}\right) C_{0}^{-1},
\end{aligned}
$$

for every $2 \leq i \leq l$ and $0 \leq j \leq l-1$. This implies

$$
\begin{aligned}
& C_{0} \mu_{t}\left(u_{\hat{l}+1-i}^{-1}\right) C_{0}^{-1}=C_{0} \mu_{t}\left(u_{l-(\hat{l}+i+1)+2}^{-1}\right) C_{0}^{-1}=u_{\hat{l}+i+1}, \\
& C_{0} \mu_{t}\left(v_{\hat{l}-j}^{-1}\right) C_{0}^{-1}=C_{0} \mu_{t}\left(v_{l-(\hat{l}+j+1)+1}^{-1}\right) C_{0}^{-1}=v_{\hat{l}+j+1} .
\end{aligned}
$$

where $0 \leq i \leq \hat{l}-1$ and $0 \leq j \leq \hat{l}-1$ Note that

$$
\mu_{t}\left(C_{0}^{-1} u_{\hat{l}+1}\right)=C_{0} \mu_{t}\left(u_{\hat{l}+1}\right)=u_{\hat{l}+1}^{-1} C_{0}=\left(C_{0}^{-1} u_{\hat{l}+1}\right)^{-1} .
$$


Since $C_{0}^{-1} u_{\hat{l}+1} \in H^{+}$, Theorem 4.2.12 implies that there exists $\hat{u} \in H$ and $s, n \in \mathbb{Z}$ such that

$$
C_{0}^{-1} u_{\hat{l}+1}=\hat{u} \xi_{n}^{s}(t+1) \mu_{t}\left(\hat{u}^{-1}\right) .
$$

Also, setting

$$
u=u_{1} v_{1} \cdot \ldots \cdot u_{\hat{l}} v_{\hat{l}} C_{0} \hat{u}
$$

gives us

$$
\begin{aligned}
\mu_{t}\left(u^{-1}\right) & =\mu_{t}\left(\hat{u}^{-1} C_{0}^{-1} v_{\hat{l}}^{-1} u_{\hat{l}}^{-1} \cdot \ldots \cdot v_{1}^{-1} u_{1}^{-1}\right) \\
& =\mu_{t}\left(\hat{u}^{-1}\right)\left[C_{0} \mu_{t}\left(v_{\hat{l}}^{-1}\right) C_{0}^{-1}\right]\left[C_{0} \mu_{t}\left(u_{\hat{l}}^{-1}\right) C_{0}^{-1}\right] \cdot \ldots \cdot\left[C_{0}^{-1} \mu_{t}\left(v_{1}^{-1}\right) C_{0}^{-1}\right]\left[C_{0} \mu_{t}\left(u_{1}^{-1}\right)\right] \\
& =\mu_{t}\left(\hat{u}^{-1}\right) v_{\hat{l}+1} u_{\hat{l}+2} \cdot \ldots \cdot u_{l} v_{l} .
\end{aligned}
$$

Therefore

$$
\begin{aligned}
u \xi_{n}^{s}(t+1) \mu_{t}\left(u^{-1}\right) & =\left(u_{1} v_{1} \cdot \ldots \cdot u_{\hat{l}} v_{\hat{l}} C_{0} \hat{u}\right) \xi_{n}^{s}(t+1)\left(\mu_{t}\left(\hat{u}^{-1}\right) v_{\hat{l}+1} u_{\hat{l}+2} \cdot \ldots \cdot u_{l} v_{l}\right) \\
& =\left(u_{1} v_{1} \cdot \ldots \cdot u_{\hat{l}} v_{\hat{l}} C_{0}\right)\left(\hat{u} \xi_{n}^{s}(t+1) \mu_{t}\left(\hat{u}^{-1}\right)\right)\left(v_{\hat{l}+1} u_{\hat{l}+2} \cdot \ldots \cdot u_{l} v_{l}\right) \\
& =\left(u_{1} v_{1} \cdot \ldots \cdot u_{\hat{l}} v_{\hat{l}} C_{0}\right)\left(C_{0} u_{\hat{l}+1}\right)\left(v_{\hat{l}+1} u_{\hat{l}+2} \cdot \ldots \cdot u_{l} v_{l}\right) \\
& =u_{1} v_{1} \cdot \ldots \cdot u_{l} v_{l} \\
& =w .
\end{aligned}
$$

For the converse, it is enough to put together Observation 4.2.11 and Corollary 4.2.10.1.

Theorem 4.2.14. Let $w_{1}, w_{2} \in H$ with $\mu_{2 r_{2}+1}\left(w_{2}\right)=w_{2}^{-1}$ satisfying equation (4.11), i.e.

$$
\mu_{r}\left(w_{1}\right)=\eta\left(w_{2}^{-1}\right) w_{1}^{-1} w_{2} .
$$

Then there are words $u_{1}, u_{2} \in H$ and integers $s_{1}, s_{2}, n_{1}, n_{2}$ such that

$$
\begin{aligned}
& w_{1}=\left[u_{2} \xi_{n_{2}}^{s_{2}}\left(2 r_{2}+2\right) \mu_{2 r_{2}+1}\left(u_{2}^{-1}\right)\right]\left[u_{1} \xi_{n_{1}}^{s_{1}}(r+1) \mu_{r}\left(u_{1}^{-1}\right)\right] \\
& w_{2}=u_{2} \xi_{n_{2}}^{s_{2}}\left(2 r_{2}+2\right) \mu_{2 r_{2}+1}\left(u_{2}^{-1}\right) .
\end{aligned}
$$

Conversely if $w_{1}$ and $w_{2}$ are given this way, then $\mu_{2 r_{2}+1}\left(w_{2}\right)=w_{2}^{-1}$ and Equation (4.11) holds.

Proof. The solutions of the equation $\mu_{2 r_{2}+1}\left(w_{2}\right)=w_{2}^{-1}$ are given by Theorem 4.2.13. There exists a word $u_{2} \in H$ and integers $s_{2}, n_{2}$ such that

$$
w_{2}=u_{2} \xi_{n_{2}}^{s_{2}}\left(2 r_{2}+2\right) \mu_{2 r_{2}+1}\left(u_{2}^{-1}\right) .
$$

Also from Proposition 4.2.2 we know that Equation (4.11) implies that $\mu_{r}\left(w_{2}^{-1} w_{1}\right)=\left(w_{2}^{-1} w_{1}\right)^{-1}$. Therefore there is a word $u_{1} \in H$ and integers $s_{1}, n_{1}$ such that

$$
w_{2}^{-1} w_{1}=u_{1} \xi_{n_{1}}^{s_{1}}(r+1) \mu_{r}\left(u_{1}^{-1}\right) .
$$

Consequently

$$
w_{1}=\left[u_{2} \xi_{n_{2}}^{s_{2}}\left(2 r_{2}+2\right) \mu_{2 r_{2}+1}\left(u_{2}^{-1}\right)\right]\left[u_{1} \xi_{n_{1}}^{s_{1}}(r+1) \mu_{r}\left(u_{1}^{-1}\right)\right] .
$$

The converse follows from the same arguments.

Thus, we solved equation (4.11) in case that $w_{2} \mu_{t}\left(w_{2}\right)=1$, now let's consider the case where $w_{2} \mu_{t}\left(w_{2}\right)$ is not the empty word. 


\subsubsection{Case $w_{2} \mu_{2 r_{2}+1}\left(w_{2}\right) \neq 1$}

Suppose that $w_{1}, w_{2} \in H$ are words such that $w_{2} \mu_{2 r_{2}+1}\left(w_{2}\right)$ is not the empty word and equation (4.11) holds, i.e.

$$
\mu_{r}\left(w_{1}\right)=\eta\left(w_{2}^{-1}\right) w_{1}^{-1} w_{2}
$$

If we set

$$
v=w_{2} \mu_{2 r_{2}+1}\left(w_{2}\right)
$$

then, by Lemma 4.2.3, the following system of equations holds in $H$, consisting of Equations (4.16) and (4.17)

$$
\begin{aligned}
\eta(v) & =w_{1}^{-1} v w_{1}, \\
\mu_{2 r_{2}+1}(v) & =w_{2}^{-1} v w_{2},
\end{aligned}
$$

where $v \neq 1$. If we solve this system of equations we obtain necessary conditions for $w_{1}$ and $w_{2}$ satisfying equation (4.11). The first step we do is to figure out which words $v$ are conjugate to $\eta(v)$, their image under $\eta$. Then we can fix such a word $v$ and give a family of words $w_{1}$ such that equation (4.16) is satisfied and find a family of words $w_{2}$ such that equation (4.16) holds. But actually it is possible to restrict $v$ even further if we make a small correction in the end.

Observation 4.2.15. a) Suppose that the triple $\left(w_{1}, w_{2}, v\right)$ satisfies the system of equations given by Equation (4.16) and Equation (4.17), i.e.

$$
\begin{array}{r}
\eta(v)=w_{1}^{-1} v w_{1} \\
\mu_{2 r_{2}+1}(v)=w_{2}^{-1} v w_{2} .
\end{array}
$$

Let $u \in H$ and set

$$
\begin{aligned}
\hat{v} & =u^{-1} v u \\
\hat{w}_{1} & =u^{-1} w_{1} \eta(u) \\
\hat{w}_{2} & =u^{-1} w_{2} \mu_{2 r_{2}+1}(u) .
\end{aligned}
$$

Then the triple $\left(\hat{w}_{1}, \hat{w}_{2}, \hat{v}\right)$ fulfils this system as well. Indeed

$$
\begin{aligned}
\eta(\hat{v}) & =\eta\left(u^{-1} v u\right)=\eta\left(u^{-1}\right)\left(w_{1}^{-1} v w_{1}\right) \eta(u)=\left(\eta\left(u^{-1}\right) w_{1}^{-1} u\right)\left(u^{-1} v u\right)\left(u^{-1} w_{1} \eta(u)\right) \\
& =\hat{w}_{1}^{-1} \hat{v} \hat{w}_{1} . \\
\mu_{2 r_{2}+1}(\hat{v}) & =\mu_{2 r_{2}+1}\left(u^{-1} v u\right)=\mu_{2 r_{2}+1}\left(u^{-1}\right)\left(w_{2}^{-1} v w_{2}\right) \mu_{2 r_{2}+1}(u) \\
& =\left(\mu_{2 r_{2}+1}\left(u^{-1}\right) w_{2}^{-1} u\right)\left(u^{-1} v u\right)\left(u^{-1} w_{2} \mu_{2 r_{2}+1}(u)\right)=\hat{w}_{2}^{-1} \hat{v} \hat{w}_{2} .
\end{aligned}
$$

Consider the family of all solutions $\left(w_{1}, w_{2}, v\right)$ of the system of equations given by Equation (4.16) and Equation (4.17) where $v$ is cyclically reduced. The family $\left(\hat{w}_{1}, \hat{w}_{2}, \hat{v}\right)$ given as above for any $u \in H$ includes every solution of the system. Therefore we can suppose that $v$ is a cyclically reduced word and in the end consider not only $w_{1}$ and $w_{2}$, but also $\hat{w}_{1}=u^{-1} w_{1} \eta(u)$ and $\hat{w}_{2}=u^{-1} w_{2} \mu_{2 r_{2}+1}(u)$ for any $u \in H$.

b) Let $v \in H$ and $t \in \mathbb{Z}$. Then the triple $\left(w_{1}, w_{2}, v\right)$ is a solution of the system of equations (4.16) and (4.17) if and only if the triple $\left(w_{1}, w_{2}, v^{t}\right)$ is. This follows from the fact that the $t$-th root in the free group $H$ is unique. Therefore if $\left(w_{1}, w_{2}, v\right)$ is a family of solutions, with $v$ being a primitive word the family $\left(w_{1}, w_{2}, v^{t}\right)$, for any $t \in \mathbb{Z}$ includes every solution of the system.

Following this observation the plan is to find the family of triples of solutions $\left(w_{1}, w_{2}, v\right)$ with $v$ a primitive cyclically reduced word and then consider the family of triples given by 
$\left(u^{-1} w_{1} \eta(u), u^{-1} w_{2} \mu_{2 r_{2}+1}(u), v^{t}\right)$, for any $u \in H$ and $t \in \mathbb{Z}$. We solve this system of equations in three parts, first we determine for which cyclically reduced words $v$ in $H, \eta(v)$ and $v$ are conjugate, then we find pairs of solutions of Equation (4.16) to then study (4.17). In the end we set the family of triples of solutions in the original equation, i.e. Equation (4.11).

We already know one family of solution of Equation (4.16), that is the pair $\left(C_{n}, \zeta_{n}\left(2 r_{1}\right)\right)$ for $n \in \mathbb{Z}$. This follows from $\eta\left(C_{n}\right)=\zeta_{n}\left(2 r_{1}\right) C_{n} \zeta_{n}\left(2 r_{1}\right)^{-1}$, which is shown in Lemma 4.2.7. The next proposition extends the family of solutions a bit.

Proposition 4.2.16. Let $n$ be a non-zero integer, $v \in\left\langle C_{n-\operatorname{sgn}(n)}, C_{n}\right\rangle$, then

$$
\eta(v)=\zeta_{n}\left(2 r_{1}\right) v \zeta_{n}^{-1}\left(2 r_{1}\right) .
$$

Proof. We know that $\eta\left(C_{n}\right)$ is a conjugate of $C_{n}$ by $\zeta_{n}\left(2 r_{1}\right)$, i.e.

$$
\eta\left(C_{n}\right)=\zeta_{n}\left(2 r_{1}\right) C_{n} \zeta_{n}^{-1}\left(2 r_{1}\right) .
$$

Actually $\eta\left(C_{n-\operatorname{sgn}(n)}\right)$ is a conjugate of $C_{n-\operatorname{sgn}(n)}$ by $\zeta_{n}\left(2 r_{1}\right)$ too, then the word $v$. For $n=-1$ this is straightforward to see, indeed, since $\zeta_{1}\left(2 r_{1}\right)=1$ it holds

$$
\eta\left(C_{n-\operatorname{sgn}(n)}\right)=\eta\left(C_{0}\right)=C_{0}=\zeta_{-1}\left(2 r_{1}\right) C_{0} \zeta_{-1}^{-1}\left(2 r_{1}\right)=\zeta_{n}\left(2 r_{1}\right) C_{n-\operatorname{sgn}(n)} \zeta_{n}^{-1}\left(2 r_{1}\right) .
$$

For $n \in \mathbb{Z} \backslash\{0,-1\}$ we can use Proposition 4.2 .8 , which states, for every $t \in \mathbb{Z}$ that

$$
\zeta_{n-\operatorname{sgn}(n)}(t) C_{n-\operatorname{sgn}(n)}^{\operatorname{sgn}(n) t}=\zeta_{n}(t)
$$

Also every letter commutes with itself, so

$$
\begin{aligned}
\eta\left(C_{n-\operatorname{sgn}(n)}\right) & =\zeta_{n-\operatorname{sgn}(n)}\left(2 r_{1}\right) C_{n-\operatorname{sgn}(n)} \zeta_{n-\operatorname{sgn}(n)}^{-1}\left(2 r_{1}\right) \\
& =\zeta_{n-\operatorname{sgn}(n)}\left(2 r_{1}\right) C_{n-\operatorname{sgn}(n)}^{\operatorname{sgn}(n) 2 r_{1}} C_{n-\operatorname{sgn}(n)} C_{n-\operatorname{sgn}(n)}^{-\operatorname{sgn}(n) 2 r_{1}} \zeta_{n-\operatorname{sgn}(n)}^{-1}\left(2 r_{1}\right) \\
& =\zeta_{n}\left(2 r_{1}\right) C_{n-\operatorname{sgn}(n)} \zeta_{n}^{-1}\left(2 r_{1}\right) .
\end{aligned}
$$

Therefore for a word $v=\prod_{i=1}^{l} C_{n-\operatorname{sgn}(n)}^{x_{i}} C_{n}^{y_{i}}$ in $\left\langle C_{n-\operatorname{sgn}(n)}, C_{n}\right\rangle$, the word $\eta(v)$ is a conjugate of $v$, namely

$$
\begin{aligned}
\eta(v) & =\prod_{i=1}^{l} \eta\left(C_{n-\operatorname{sgn}(n)}^{x_{i}}\right) \eta\left(C_{n}^{y_{i}}\right) \\
& \left.=\prod_{i=1}^{l}\left(\zeta_{n}\left(2 r_{1}\right) C_{n-\operatorname{sgn}(n)} \zeta_{n}^{-1}\left(2 r_{1}\right)\right)^{x_{i}}\left(\zeta_{n}\left(2 r_{1}\right) C_{n} \zeta_{n}^{-1}\left(2 r_{1}\right)\right)^{y_{i}}\right) \\
& =\zeta_{n}\left(2 r_{1}\right)\left(\prod_{i=1}^{l} C_{n-\operatorname{sgn}(n)}^{x_{i}} C_{n}^{y_{i}}\right) \zeta_{n}^{-1}\left(2 r_{1}\right) \\
& =\zeta_{n}\left(2 r_{1}\right) v \zeta_{n}^{-1}\left(2 r_{1}\right) .
\end{aligned}
$$

Let $F$ be a free group and let $v_{1}, v_{2}$ be cyclically reduced words in $F$. The conjugacy problem in free groups is solvable, see for example Combinatorial Group Theory from Lyndon and Schupp [LS15]. There it states that if the words $v_{1}$ and $v_{2}$ are conjugates, then $v_{1}$ is a cyclic permutation of $v_{2}$.

Let $G_{1}$ and $G_{2}$ be two subgroups of $F$ with $G_{1} \cap G_{2}=\{1\}$ and suppose that $v_{1}, v_{2}$ are conjugate words in the free product $G_{1} * G_{2}$ such that their reduced form begin with a non-trivial letter in $G_{1}$ 
and end with a non-trivial letter in $G_{2}$. Then it is possible to write $v_{1}$ and $v_{2}$ in the following way

$$
\begin{aligned}
& v_{1}=\prod_{i=1}^{l_{1}} p_{i} q_{i} \\
& v_{2}=\prod_{j=1}^{l_{2}} \hat{p}_{j} \hat{q}_{j},
\end{aligned}
$$

where $p_{i}, \hat{p}_{j} \in G_{1}, q_{i}, \hat{q}_{j} \in G_{2}$ are non-empty words for $1 \leq i \leq l_{1}$ and $1 \leq j \leq l_{2}$. These are not arbitrary words in the free product $G_{1} * G_{2}$, as they both begin with a letter in $G_{1}$ and end with a letter in $G_{2}$. Since $v_{1}$ and $v_{2}$ are conjugates, $v_{1}$ is a cyclic permutation of $v_{2}$, therefore $l_{1}=l_{2}$ and we will denote it by $l$. Thus, there is an integer $k \in\{1, \ldots, l\}$ such that

$$
\prod_{i=1}^{l} p_{i} q_{i}=\left(\prod_{j=k}^{l} \hat{p}_{j} \hat{q}_{j}\right)\left(\prod_{j=1}^{k-1} \hat{p}_{j} \hat{q}_{j}\right)
$$

Define a permutation $\sigma \in \mathcal{S}_{l}$ in the following way:

$$
\begin{aligned}
\sigma(1) & =k, \\
\sigma(2) & =k+1, \\
\cdots & \\
\sigma(l-k) & =l, \\
\sigma(l-k+1) & =1, \\
\sigma(l) & =k-1 .
\end{aligned}
$$

Hence this permutation satisfies $p_{i}=\hat{p}_{\sigma(i)}$ and $q_{i}=\hat{q}_{\sigma(i)}$ for $1 \geq i \geq l$. It is possible to define $\sigma$ the following way: $\sigma(i)$ is the integer in $\{1, \ldots l\}$ that is congruent to $\sigma(1)+i-1$ modulo $l$.

Proposition 4.2.17. The following properties hold for $\sigma$ defined as above, where $k \in \mathbb{N}$.

a) For $1 \leq i \leq l$ it holds that $\sigma^{k}(i)$ is congruent to $k \sigma(1)-k+i$ modulo $l$.

b) For $1 \leq i \leq l$ it holds that $\sigma^{k}(i) \in\{1, \ldots l\}$ is congruent to $\sigma^{k}(1)+i-1$ modulo $l$.

c) The order of $\sigma$ is given by the smallest integer $\lambda \in \mathbb{N}$ such that $\sigma^{\lambda}(1)=1$, and all cycles of the permutation have the same length.

Proof. a) To show this by induction note that this holds for $k=1$, by definition of $\sigma$. Suppose that

$$
\sigma^{k}(i) \equiv k \sigma(1)-k+i(\bmod l) .
$$

Then

$$
\begin{aligned}
\sigma^{k+1}(i) & =\sigma\left(\sigma^{k}(i)\right) \\
& \equiv \sigma(1)+\left(\sigma^{k}(i)\right)-1 \\
& \equiv \sigma(1)+(k \sigma(1)-k+i)-1 \\
& =(k+1) \sigma(1)-(k+1)+i(\bmod l) .
\end{aligned}
$$

b) Note that

$$
\sigma^{k}(1) \equiv k \sigma(1)-k+1(\bmod l)
$$


Hence

$$
\begin{aligned}
\sigma^{k}(i) & \equiv k \sigma(1)-k+i \\
& =(k \sigma(1)-k+1)+i-1 \\
& \equiv \sigma^{k}(1)+i-1(\bmod l) .
\end{aligned}
$$

c) There is an integer $\lambda \in \mathbb{N}$ with $\sigma^{\lambda}(1)=1$. Thus for every $i \in\{1, \ldots, l\}$ it follows from the previous items that

$$
\sigma^{\lambda}(i) \equiv \sigma^{\lambda}(1)+i-1 \equiv i(\bmod l)
$$

Therefore $\lambda$ is the order of $\sigma$.

It is useful, that every word $v \in G_{1} * G_{2}$ is conjugate to a word with that form.

Proposition 4.2.18. Let $G_{1}, G_{2}$ be subgroups such that $G_{1} \cap G_{2}=\{1\}$ of the free group $F$ and let $v \in G_{1} * G_{2}$ be cyclically reduced word in the free product of $G_{1}$ and $G_{2}$. Then there is a word $\hat{v}$, conjugate to $v$ with the form

$$
\hat{v}=\prod_{i=1}^{l} p_{i} q_{i},
$$

where $p_{i} \in G_{1}, q_{i} \in G_{2}$ are non-empty words for $1 \leq i \leq l$.

Proof. Since $v \in G_{1} * G_{2}$, the word $v$ can be written in the form

$$
\hat{v}=\prod_{i=1}^{l} p_{i} q_{i},
$$

where $p_{i} \in G_{1}, q_{i} \in G_{2}$ and $p_{2}, \ldots, p_{l}, q_{1}, \ldots, q_{l-1}$ are non-empty words.

Suppose $p_{1} \neq 1$ and $q_{l}=1$, then

$$
v=p_{1} q_{1} \cdot \ldots \cdot q_{l-1} p_{l}
$$

is conjugate to

$$
\hat{v}=\left(p_{l}^{-1} p_{1}\right) q_{1} p_{2} \cdot \ldots \cdot p_{l-1} q_{l-1}
$$

The word $p_{l}^{-1} p_{1}$ is not empty since $v$ is cyclically reduced. Hence we obtained a word $\hat{v}$ in the wished form which is conjugate to $v$. This procedure is similar in the four cases, depending if $p_{1}$ or $q_{l}$ are the empty word or not and we will not write the other cases here.

Note that if $v \in H \subset F_{2}$ and $\hat{v}$ are conjugates, then $v$ and $\eta(v)$ are conjugates if and only if $\hat{v}$ and $\eta(\hat{v})$ are conjugates too.

In the next proposition we display all words $v$ in $H$ that are conjugates to their image under $\eta^{\kappa}$, for an integer $\kappa$. Actually our original goal was to find the conjugates with $\kappa$ equal to one, but due to technical reasons we take $\kappa$ any integer.

Proposition 4.2.19. Let $\kappa \in \mathbb{N}$ and $v \in H$ be a cyclically reduced word such that $\eta^{\kappa}(v)$ and $v$ are conjugates, then there is a non-zero integer $n \in \mathbb{Z}$ such that $v \in\left\langle C_{n-\operatorname{sgn}(n)}, C_{n}\right\rangle$. The converse holds as well. 
Proof. If $v$ is a power of an element $C_{n}$ of the basis $\mathcal{B}=\left\{C_{n}: n \in \mathbb{Z}\right\}$, then there is nothing to prove. Suppose that $v \in H$ a cyclically reduced word that is not a power of a letter $C_{n}$. Then there are integers $n_{1}, n_{2} \in \mathbb{Z}$ with $n_{1}<n_{2}$ such that $v \in\left\langle C_{n_{1}}, C_{n_{1}+1}, \ldots, C_{n_{2}}\right\rangle$ and $v \notin\left\langle C_{n_{1}+1}, \ldots, C_{n_{2}}\right\rangle$ as well as $v \notin\left\langle C_{n_{1}}, \ldots, C_{n_{2}-1}\right\rangle$ i.e. $C_{n_{1}}$ and $C_{n_{2}}$ appear as letters in $v$.

Then, if $n_{2} \geq 1$, set $n=n_{2}$ and $\hat{n}=n_{1}$, otherwise let $n=n_{1}$ and $\hat{n}=n_{2}$. This way $n \neq 0$, since if $n_{2} \leq 0$ it follows that $t=n_{1}<n_{2} \leq 0$. Therefore it is possible to say that

$$
v \in\left\langle C_{n}, C_{n-\operatorname{sgn}(n)}, \ldots, C_{\hat{n}}\right\rangle \backslash\left(\left\langle C_{n-\operatorname{sgn}(n)}, \ldots, C_{\hat{n}}\right\rangle \cup\left\langle C_{n}, \ldots, C_{\hat{n}-\operatorname{sgn}(\hat{n})}\right\rangle\right) .
$$

We can suppose that $v$ has the form

$$
\prod_{i=1}^{l} p_{i} C_{n}^{y_{i}}
$$

with $p_{i} \in\left\langle C_{n-\operatorname{sgn}(n)}, \ldots, C_{\hat{n}}\right\rangle$ non-empty words and $y_{i} \in \mathbb{Z}$ non-zero integers. Indeed, if this is not the case, then by Proposition 4.2.18 there is a word $\hat{v}$ conjugate to $v$ with the form

$$
\hat{v}=\prod_{i=1}^{l} p_{i} C_{n}^{y_{i}},
$$

with $p_{i} \in\left\langle C_{n-\operatorname{sgn}(n)}, \ldots, C_{\hat{n}}\right\rangle$ non-empty words and $y_{i} \in \mathbb{Z}$ non-zero integers. Then $v$ and $\eta^{\kappa}(v)$ are conjugates if and only if $\hat{v}$ and $\eta^{\kappa}(\hat{v})$ are conjugates. Also, since $v$ and $\hat{v}$ are cyclically reduced conjugates and therefore cyclic permutations from one another they are contained in exactly the same subgroups of $H$.

Suppose that $v$ has the form above, as in (4.25). We show by induction, that if $|n-\hat{n}| \geq 2$, then $v$ and $\eta^{\kappa}(v)$ are not conjugates, for any $\kappa>0$.

Let $|n-\hat{n}|=2$, hence $v \in\left\langle C_{n}, C_{n-\operatorname{sgn}(n)}, C_{n-\operatorname{sgn}(n) 2}\right\rangle$. Fix $\kappa>0$ and suppose that $v$ and $\eta^{\kappa}(v)$ are conjugates. Since $p_{i} \in\left\langle C_{n-\operatorname{sgn}(n)}, C_{n-\operatorname{sgn}(n) 2}\right\rangle$ it follows from Proposition 4.2.16 that

$$
\eta\left(p_{i}\right)=\zeta_{n-\operatorname{sgn}(n)}\left(2 r_{1}\right) p_{i} \zeta_{n-\operatorname{sgn}(n)}^{-1}\left(2 r_{1}\right) .
$$

From Proposition 4.2.9 and Equation (4.19) it follows

$$
\begin{aligned}
\eta^{2}\left(p_{i}\right) & =\eta\left(\zeta_{n-\operatorname{sgn}(n)}\left(2 r_{1}\right)\right) \eta\left(p_{i}\right) \eta\left(\zeta_{n-\operatorname{sgn}(n)}^{-1}\left(2 r_{1}\right)\right) \\
& =\left[\zeta_{n-\operatorname{sgn}(n)}\left(4 r_{1}\right) \zeta_{n-\operatorname{sgn}(n)}^{-1}\left(2 r_{1}\right)\right]\left[\zeta_{n-\operatorname{sgn}(n)}\left(2 r_{1}\right) p_{i} \zeta_{n-s g n(n)}^{-1}\left(2 r_{1}\right)\right]\left[\zeta_{n-\operatorname{sgn}(n)}\left(2 r_{1}\right) \zeta_{n-\operatorname{sgn}(n)}^{-1}\left(4 r_{1}\right)\right] \\
& =\zeta_{n-\operatorname{sgn}(n)}\left(4 r_{1}\right) p_{i} \zeta_{n-\operatorname{sgn}(n)}^{-1}\left(4 r_{1}\right) .
\end{aligned}
$$

After $\kappa$ iterations we have

$$
\eta^{\kappa}\left(p_{i}\right)=\zeta_{n-\operatorname{sgn}(n)}\left(2 r_{1} \kappa\right) p_{i} \zeta_{n-\operatorname{sgn}(n)}^{-1}\left(2 r_{1} \kappa\right) .
$$

Analogously, it follows from Lemma 4.2.7 that

$$
\begin{aligned}
\eta^{\kappa}\left(C_{n}\right) & =\zeta_{n}\left(2 r_{1} \kappa\right) C_{n} \zeta_{n}^{-1}\left(2 r_{1} \kappa\right) \\
& =\left[\zeta_{n-\operatorname{sgn}(n)}\left(2 r_{1} \kappa\right) C_{n-\operatorname{sgn}(n)}^{2 r_{1} \kappa}\right] C_{n}\left[C_{n-\operatorname{sgn}(n)}^{-2 r_{1} \kappa} \zeta_{n-\operatorname{sgn}(n)}^{-1}\left(2 r_{1} \kappa\right)\right] .
\end{aligned}
$$


Therefore

$$
\begin{aligned}
& \eta^{\kappa}(v)=\prod_{i=1}^{l}\left[\eta^{\kappa}\left(p_{i}\right) \eta^{\kappa}\left(C_{n}^{y_{i}}\right)\right] \\
& =\prod_{i=1}^{l}\left[\zeta_{n-\operatorname{sgn}(n)}\left(2 r_{1} \kappa\right) p_{i} \zeta_{n-\operatorname{sgn}(n)}^{-1}\left(2 r_{1} \kappa\right)\right]\left[\zeta_{n-\operatorname{sgn}(n)}\left(2 r_{1} \kappa\right) C_{n-\operatorname{sgn}(n)^{2 r_{1} \kappa}} C_{n}^{y_{i}} C_{n-\operatorname{sgn}(n)}^{-2 r_{1} \kappa} \zeta_{n-\operatorname{sgn}(n)}^{-1}\left(2 r_{1} \kappa\right)\right] \\
& =\prod_{i=1}^{l}\left[\zeta_{n-\operatorname{sgn}(n)}\left(2 r_{1} \kappa\right)\right] p_{i} C_{n-\operatorname{sgn}(n)^{2 r_{1} \kappa}} C_{n}^{y_{i}}\left[C_{n-\operatorname{sgn}(n)}^{-2 r_{1} \kappa} \zeta_{n-s g n(n)}^{-1}\left(2 r_{1} \kappa\right)\right] \\
& =\zeta_{n-\operatorname{sgn}(n)}\left(2 r_{1} \kappa\right)\left(C_{n-\operatorname{sgn}(n)^{2 r_{1} \kappa}}\left[\prod_{i=1}^{l} C_{n-\operatorname{sgn}(n)}^{-2 r_{1} \kappa} p_{i} C_{n-\operatorname{sgn}(n)}^{2 r_{1} \kappa} C_{n}^{y_{i}}\right] C_{n-\operatorname{sgn}(n)}^{-2 r_{1} \kappa} \zeta_{n-s g n(n)}^{-1}\left(2 r_{1} \kappa\right)\right.
\end{aligned}
$$

Since $p_{i}$ is non-empty and $C_{n-\operatorname{sgn}(n)}^{-2 r_{1} \kappa} p_{i} C_{n-\operatorname{sgn}(n)}^{2 r_{1} \kappa} \in\left\langle C_{n-\operatorname{sgn}(n)}, C_{n-\operatorname{sgn}(n) 2}\right\rangle$ the cyclic reduction of $\eta^{\kappa}(v)$ is

$$
\hat{v}=\prod_{i=1}^{l} C_{n-\operatorname{sgn}(n)}^{-2 r_{1} \kappa} p_{i} C_{n-\operatorname{sgn}(n)}^{2 r_{1} \kappa} C_{n}^{y_{i}} .
$$

Now $v$ and $\eta^{k}(v)$ are conjugates if and only if $v$ is a cyclic permutation of $v$. Therefore, by Proposition 4.2.17, there is a permutation $\sigma$ with order $\lambda$ such that

$$
p_{i}=C_{n-\operatorname{sgn}(n)}^{-2 r_{1} \kappa} p_{\sigma}(i) C_{n-\operatorname{sgn}(n)}^{2 r_{1} \kappa} .
$$

Iterating this $\lambda$ times implies

$$
\begin{aligned}
p_{i} & =C_{n-\operatorname{sgn}(n)}^{-2 r_{1} \kappa \lambda} p_{\sigma}^{\lambda}(i) C_{n-\operatorname{sgn}(n)}^{2 r_{1} \kappa \lambda} \\
& =C_{n-\operatorname{sgn}(n)}^{-2 r_{1} \kappa \lambda} p_{i} C_{n-\operatorname{sgn}(n)}^{2 r_{1} \kappa \lambda} .
\end{aligned}
$$

Hence $p_{i}$ and $C_{n-\operatorname{sgn}(n)}^{2 r_{1} \kappa \lambda}$ commute and $p_{i} \in\left\langle C_{n-\operatorname{sgn}(n)}\right\rangle$ for all $i \in\{1, \ldots, l\}$. But this contradicts the fact that $v \notin\left\langle C_{n}, C_{n-\operatorname{sgn}(n)}\right\rangle$, i.e. that the letter $C_{\hat{n}}$ appears in $v$. Therefore $v$ and $\eta^{\kappa}(v)$ are not conjugates.

Suppose that the induction hypothesis is true for words $v$ in subgroups $\left\langle C_{n_{1}}, \ldots C_{n_{2}}\right\rangle$, that are smaller than $\left\langle C_{n}, \ldots C_{\hat{n}}\right\rangle$, i.e. $\left|n_{1}-n_{2}\right|<|n-\hat{n}|$. Let $v \in\left\langle C_{n}, \ldots, C_{\hat{n}}\right\rangle$ be a cyclically reduced word where the letters $C_{n}, C_{\hat{n}}$ appear and that is conjugate to $\eta^{\kappa}(v)$.

Applying $\eta^{\kappa}$ on $v$, using equation 4.26 the following is obtained:

$$
\begin{aligned}
\eta^{\kappa}(v) & =\prod_{i=1}^{l}\left[\eta^{\kappa}\left(p_{i}\right) \eta^{\kappa}\left(C_{n}^{k_{i}}\right)\right] \\
& =\prod_{i=1}^{l} \eta^{\kappa}\left(p_{i}\right)\left[\zeta_{n}\left(2 r_{1} \kappa\right) C_{n}^{k_{i}} \zeta_{n}^{-1}\left(2 r_{1} \kappa\right)\right] \\
& =\zeta_{n}\left(2 r_{1} \kappa\right)\left[\prod_{i=1}^{l}\left(\zeta_{n}^{-1}\left(2 r_{1} \kappa\right) \eta^{\kappa}\left(p_{i}\right) \zeta_{n}\left(2 r_{1} \kappa\right)\right) C_{n}^{k_{i}}\right] \zeta_{n}^{-1}\left(2 r_{1} \kappa\right)
\end{aligned}
$$

Note that $\zeta_{n}\left(2 r_{1} \kappa\right), p_{i} \in\left\langle C_{n-\operatorname{sgn}(n)}, C_{n-2 s g n(n)}, \ldots\right\rangle$, thus $\zeta_{n}^{-1}\left(2 r_{1} \kappa\right) \eta^{\kappa}\left(p_{i}\right) \zeta_{n}\left(2 r_{1} \kappa\right) \in H \backslash\left\langle C_{n}\right\rangle$. Hence, since $\zeta_{n}^{-1}\left(2 r_{1}\right) \eta^{\kappa}\left(p_{i}\right) \zeta_{n}\left(2 r_{1}\right)$ is not the empty word and $y_{i} \neq 0$, the word

$$
\hat{v}=\prod_{i=1}^{l}\left[\zeta_{n}^{-1}\left(2 r_{1} \kappa\right) \eta\left(p_{i}\right) \zeta_{n}\left(2 r_{1} \kappa\right) C_{n}^{k_{i}}\right]
$$


is cyclically reduced and conjugate to $\eta^{\kappa}(v)$ and therefore also to $v$.

Then there is a permutation $\sigma \in \mathcal{S}_{l}$, such that for every $i\{1, \ldots, l\}$ it holds that

$$
p_{i}=\zeta_{n}^{-1}\left(2 r_{1}\right) \eta^{\kappa}\left(p_{\sigma(i)}\right) \zeta_{n}\left(2 r_{1}\right) .
$$

Therefore, for an integer $m \in \mathbb{Z}$

$$
p_{\sigma^{m}}(i)=\zeta_{n}^{-1}\left(2 r_{1}\right) \eta^{\kappa}\left(p_{\sigma^{m+1}(i)}\right) \zeta_{n}\left(2 r_{1}\right) .
$$

Let $\lambda$ be the order of $\sigma$, then after $\lambda$ iterations we obtain

$$
\begin{aligned}
p_{i} & =\zeta_{n}^{-1}\left(2 r_{1}\right) \eta^{\kappa}\left(p_{\sigma(i)}\right) \zeta_{n}\left(2 r_{1}\right) \\
& =\left[\zeta_{n}^{-1}\left(2 r_{1}\right) \eta^{\kappa}\left(\zeta_{n}^{-1}\left(2 r_{1}\right)\right)\right] \eta^{2 \kappa}\left(p_{\text {sigma }}(i)\right)\left[\eta^{\kappa}\left(\zeta_{n}\left(2 r_{1}\right)\right) \zeta_{n}\left(2 r_{1}\right)\right] \\
& \ldots \\
& =\left[\zeta_{n}^{-1}\left(2 r_{1}\right) \ldots \eta^{(\lambda-1) \kappa}\left(\zeta_{n}^{-1}\left(2 r_{1}\right)\right)\right] \eta^{\lambda \kappa}\left(p_{\text {sigma }^{\lambda}(i)}\right)\left[\eta^{(\lambda-1) \kappa}\left(\zeta_{n}\left(2 r_{1}\right)\right) \ldots \zeta_{n}\left(2 r_{1}\right)\right] \\
& =\left[\zeta_{n}^{-1}\left(2 r_{1}\right) \ldots \eta^{(\lambda-1) \kappa}\left(\zeta_{n}^{-1}\left(2 r_{1}\right)\right)\right] \eta^{\lambda \kappa}\left(p_{i}\right)\left[\eta^{(\lambda-1) \kappa}\left(\zeta_{n}\left(2 r_{1}\right)\right) \ldots \zeta_{n}\left(2 r_{1}\right)\right] .
\end{aligned}
$$

Therefore for all $i$ the word $p_{i} \in\left\langle C_{n-\operatorname{sgn}(n)}, \ldots, C_{\hat{n}}\right\rangle$ is a conjugate to $\eta^{\lambda \kappa}\left(p_{i}\right)$. But $|n-\operatorname{sgn}(n)-\hat{n}|<$ $|n-\hat{n}|$, from the way $n$ and $\hat{n}$ are defined, which by hypothesis of induction means that $p_{i}$ and $\eta^{\lambda \kappa}\left(p_{i}\right)$ are not conjugates, a contradiction.

Now that we know when a word and its image under $\eta$ are conjugates we can solve Equation (4.16).

Proposition 4.2.20. Let $w_{1}, v \in H$ with $v$ a cyclically reduced primitive word such that equation (4.16) holds, i.e.

$$
\eta(v)=w_{1}^{-1} v w_{1},
$$

then there exist integers $n, \rho_{1} \in \mathbb{Z}, n \neq 0$ such that $v \in\left\langle C_{n-\operatorname{sgn}(n)}, C_{n}\right\rangle$ and

$$
w_{1}=v^{\rho_{1}} \zeta_{n}^{-1}\left(2 r_{1}\right)
$$

Proof. Equation (4.16) implies that $\eta(v)$ and $v$ are conjugates. From Proposition 4.2.19 there exists a non-zero integer $n$ such that $v \in\left\langle C_{n}, C_{n-\operatorname{sgn}(n)}\right\rangle$. Proposition 4.2.16 implies

$$
\eta(v)=\zeta_{n}\left(2 r_{1}\right) v \zeta_{n}^{-1}\left(2 r_{1}\right) .
$$

Now set $q=w_{1} \zeta_{n}\left(2 r_{1}\right)$, then $q$ and $v$ commute. Indeed, using the equations (4.16) and (4.24) it follows

$$
\begin{aligned}
q v q^{-1} & =w_{1}\left[\zeta_{n}\left(2 r_{1}\right) v \zeta_{n}^{-1}\left(2 r_{1}\right)\right] w_{1}^{-1} \\
& =w_{1} \eta(v) w_{1}^{-1} \\
& =v
\end{aligned}
$$

Since $v$ is a primitive word, this implies that there is an integer $\rho_{1} \in \mathbb{Z}$ such that $q=v_{1}^{\rho}$. Therefore

$$
w_{1}=q \zeta_{n}^{-1}\left(2 r_{1}\right)=v^{\rho_{1}} \zeta_{n}^{-1}\left(2 r_{1}\right)
$$

The next equation to be solved is equation (4.17), i.e.

$$
\mu_{2 r_{2}+1}(v)=w_{2}^{-1} v w_{2},
$$


for $v \in\left\langle C_{n}, C_{n-\operatorname{sgn}(n)}\right\rangle$. For that it is useful to know how to write $\mu_{2 r_{2}+1}(v)$ in relation to $v$. For every word $v \in\left\langle C_{n}, C_{n-s g n(n)}\right\rangle$, there are integers $x_{i}, y_{i}$ such that

$$
v=\prod_{i=1}^{l} C_{n-\operatorname{sgn}(n)}^{x_{i}} C_{n}^{y_{i}}
$$

Define for every $v \in\left\langle C_{n-\operatorname{sgn}(n)}, C_{n}\right\rangle$ the following word in $\left\langle C_{n-\operatorname{sgn}(n)}, C_{n}\right\rangle$

$$
v^{-}=\prod_{i=1}^{l} C_{n-s g n(n)}^{-x_{i}} C_{n}^{-y_{i}} .
$$

Proposition 4.2.21. Let $v \in\left\langle C_{n-s g n n}, C_{n}\right\rangle$, then for every $t, n \neq 0$

$$
\mu_{t}(v)=C_{0}^{-1} \zeta_{n}(t+1) v^{-} \zeta_{n}^{-1}(t+1) C_{0} .
$$

Proof. As in the proof of Proposition 4.2.16 it is enough to show that $\mu_{t}\left(C_{n-\operatorname{sgn}(n)}\right)$ is conjugate to $C_{n-\operatorname{sgn}(n)}^{-}$by $C_{0}^{-1} \zeta_{n}(k)$, since

$$
\mu_{t}\left(C_{n}\right)=C_{0}^{-1} \zeta_{n}(t+1) C_{n}^{-1} \zeta_{n}^{-1}(t+1) C_{0},
$$

by Corollary 4.2.6.

Again, for $n=-1$ this is straightforward to see, indeed

$$
\mu_{t}\left(C_{n-s g n(n)}\right)=\mu\left(C_{0}\right)=C_{0}^{-} 1=\zeta_{-1}(t) C_{0}^{-1} \zeta_{-1}^{-1}(t)=\zeta_{n}(t) C_{n-\operatorname{sgn}(n)}^{-1} \zeta_{n}^{-1}(t) .
$$

For $n \in \mathbb{Z} \backslash\{0,-1\}$, using Proposition 4.2 .8 we get

$$
\begin{aligned}
\mu_{t}\left(C_{n-\operatorname{sgn}(n)}\right) & =C_{0}^{-1} \zeta_{n-\operatorname{sgn}(n)}(t) C_{n-\operatorname{sgn}(n)}^{-1} \zeta_{n-\operatorname{sgn}(n)}^{-1}(t) C_{0} \\
& =C_{0}^{-1} \zeta_{n-\operatorname{sgn}(n)}(t) C_{n-\operatorname{sgn}(n)}^{\operatorname{sgn}(n) k} C_{n-\operatorname{sgn}(n)}^{-1} C_{n-\operatorname{sgn}(n)}^{-\operatorname{sgn}(n) k} \zeta_{n-\operatorname{sgn}(n)}^{-1}(t) C_{0} \\
& =C_{0}^{-1} \zeta_{n}(t) C_{n-\operatorname{sgn}(n)} \zeta_{n}^{-1}(t) C_{0} .
\end{aligned}
$$

Therefore for a word $v=\prod_{i=1}^{l} C_{n-\operatorname{sgn}(n)}^{x_{i}} C_{n}^{y_{i}}$ in $\left\langle C_{n-\operatorname{sgn}(n)}, C_{n}\right\rangle$, the word $\mu_{t}(v)$ is plainly a conjugate of $v^{-}$,

$$
\begin{aligned}
\mu_{t}(v) & =\prod_{i=1}^{l} \mu_{t}\left(C_{n-s g n(n)}^{x_{i}} C_{n}^{y_{i}}\right) \\
& =C_{0}^{-1} \zeta_{n}(t)\left(\prod_{i=1}^{l} C_{n-s g n(n)}^{-x_{i}} C_{n}^{-y_{i}}\right) \zeta_{n}^{-1}\left(2 r_{1}\right) C_{0} \\
& =C_{0}^{-1} \zeta_{n}\left(2 r_{1}\right) v^{-} \zeta_{n}^{-1}\left(2 r_{1}\right) C_{0} .
\end{aligned}
$$

Corollary 4.2.21.1. Let $w_{2} \in H$, let $n \in \mathbb{Z}$ be a integer different from zero and let $v \in\left\langle C_{n}, C_{n-\operatorname{sgn}(n)}\right\rangle$ such that

$$
\mu_{2 r_{2}+1}(v)=w_{2}^{-1} v w_{2} .
$$

then $v$ and $v^{-}$are conjugate words. 
Proof. This follows from

$$
w_{2}^{-1} v w_{2}=\mu_{2 r_{2}+1}(v)=C_{0}^{-1} \zeta_{n}(t+1) v^{-} \zeta_{n}^{-1}(t+1) C_{0} .
$$

Proposition 4.2.22. Let $w_{2} \in H, n \in \mathbb{Z}$ be a non-zero integer and let $v \in\left\langle C_{n}, C_{n-\operatorname{sgn}(n)}\right\rangle$ a primitive, cyclically reduced word with $n \in \mathbb{Z}$, such that

$$
\mu_{2 r_{2}+1}(v)=w_{2}^{-1} v w_{2} .
$$

Then there exists a word $p \in\left\langle C_{n}, C_{n-\operatorname{sgn}(n)}\right\rangle$ and an integer $\rho_{2} \in \mathbb{Z}$ such that

$$
\begin{aligned}
v & =p p^{-}, \\
w_{2} & =\left(p p^{-}\right)^{\rho_{2}} p \zeta_{n}^{-1}\left(2 r_{2}+2\right) C_{0} .
\end{aligned}
$$

Proof. By Corollary 4.2.21.1 $v$ and $v^{-}$are conjugated and by [GG18, Lemma 33(b)] there exists $p \in\left\langle C_{n}, C_{n-\operatorname{sgn}(n)}\right\rangle$ such that $v=p p^{-}$. Since $p p^{-} \in\left\langle C_{n}, C_{n-s g n(n)}\right\rangle$ we can use Proposition 4.2.21. Also $\left(p p^{-}\right)^{-}=p^{-} p$, thus

$$
\begin{aligned}
\mu_{2 r_{2}+1}(v) & =\mu_{2 r_{2}+1}\left(p p^{-}\right) \\
& =\left[C_{0}^{-1} \zeta_{n}\left(2 r_{2}+2\right)\right]\left(p p^{-}\right)^{-}\left[\zeta_{n}^{-1}\left(2 r_{2}+2\right) C_{0}\right] \\
& =\left[C_{0}^{-1} \zeta_{n}\left(2 r_{2}+2\right)\right]\left(p^{-} p\right)\left[\zeta_{n}^{-1}\left(2 r_{2}+2\right) C_{0}\right] \\
& =\left[C_{0}^{-1} \zeta_{n}\left(2 r_{2}+2\right)\right] p^{-1}\left(p p^{-}\right) p\left[\zeta_{n}^{-1}\left(2 r_{2}+2\right) C_{0}\right] \\
& =\left[C_{0}^{-1} \zeta_{n}\left(2 r_{2}+2\right) p^{-1}\right] v\left[p \zeta_{n}^{-1}\left(2 r_{2}+2\right) C_{0}\right] .
\end{aligned}
$$

This gives us one solution for this equation. Set $q=w_{2} C_{0}^{-1} \zeta_{n}\left(2 r_{2}+2\right) p^{-1}$, then $q$ commutes with $v$. Indeed

$$
\begin{aligned}
q v q^{-1} & =w_{2}\left(C_{0}^{-1} \zeta_{n}\left(2 r_{2}+2\right) p^{-1} v p \zeta_{n}^{-1}\left(2 r_{2}+2\right) C_{0}\right) w_{2}^{-1} \\
& =w_{2} \mu_{2 r_{2}+1}(v) w_{2}^{-1} \\
& =v
\end{aligned}
$$

Since $v$ is a primitive word there is an integer $\rho_{2} \in \mathbb{Z}$ such that $q=v^{\rho_{2}}$. Hence

$$
\begin{aligned}
w_{2} & =q\left[p \zeta_{n}^{-1}\left(2 r_{2}+2\right) C_{0}\right] \\
& =(v)^{\rho_{2}} p \zeta_{n}^{-1}\left(2 r_{2}+2\right) C_{0} \\
& =\left(p p^{-}\right)^{\rho_{2}} p \zeta_{n}^{-1}\left(2 r_{2}+2\right) C_{0} .
\end{aligned}
$$

Theorem 4.2.23. Let $w_{1}, w_{2}$ be words in $H$ with $w_{2} \mu_{2 r_{2}+1}\left(w_{2}\right) \neq 1$.

If

$$
\mu_{r}\left(w_{1}\right)=\eta\left(w_{2}^{-1}\right) w_{1}^{-1} w_{2},
$$

then there exist integers $\rho \in \mathbb{Z}$, a non zero integer $n$, a word $u \in H$ and a word $p \in\left\langle C_{n-\operatorname{sngn}(n)}, C_{n}\right\rangle$ with $\mathrm{pp}^{-}$is a primitive cyclically reduced word such that

$$
\begin{aligned}
& w_{1}=u^{-1} \zeta_{n}^{-1}\left(2 r_{1}\right) \eta(u) \\
& w_{2}=u^{-1}\left(p p^{-}\right)^{\rho} p \zeta_{n}^{-1}\left(2 r_{2}+2\right) C_{0} \mu_{2 r_{2}+1}(u) .
\end{aligned}
$$


Conversely if

$$
\begin{aligned}
& w_{1}=u^{-1} \zeta_{n}^{-1}\left(2 r_{1}\right) \eta(u) \\
& w_{2}=u^{-1}\left(p p^{-}\right)^{\rho} p \zeta_{n}^{-1}\left(2 r_{2}+2\right) C_{0} \mu_{2 r_{2}+1}(u) .
\end{aligned}
$$

with $u, p, \rho, n$ as above, then

$$
\mu_{r}\left(w_{1}\right)=\eta\left(w_{2}^{-1}\right) w_{1}^{-1} w_{2} .
$$

Proof. From Lemma 4.2.3 we know that Equation (4.27) implies the following system of equations

$$
\begin{aligned}
\eta(v) & =w_{1}^{-1} v w_{1} \\
\mu_{2 r_{2}+1}(v) & =w_{2}^{-1} v w_{2},
\end{aligned}
$$

where $v$ is the non-empty word $w_{2} \mu_{2 r_{2}+1}\left(w_{2}\right)$. Then there is a primitive cyclically reduced word $\hat{v}$, an integer $k$ and a word $u \in H$ such that $v=u^{-1} \hat{v}^{k} u$. Also, set

$$
\begin{aligned}
& \hat{w}_{1}=u w_{1} \eta\left(u^{-1}\right) \\
& \hat{w}_{2}=u w_{2} \mu_{2 r_{2}+1}\left(u^{-1}\right) .
\end{aligned}
$$

Then by Observation 4.2.15 $a$ ) and $b$ ) it the following system of equations holds for the triple $\left(\hat{w}_{1}, \hat{w}_{2}, \hat{v}\right)$

$$
\begin{aligned}
\eta(\hat{v}) & =\hat{w}_{1}^{-1} \hat{v} \hat{w}_{1} \\
\mu_{2 r_{2}+1}(\hat{v}) & =\hat{w}_{2}^{-1} \hat{v} \hat{w}_{2},
\end{aligned}
$$

Then Proposition 4.2.20 implies that there exists integers $n, \rho_{1} \in \mathbb{Z}, n \neq 0$ such that $v \in\left\langle C_{n-\operatorname{sgn}(n)}, C_{n}\right\rangle$ and

$$
\hat{w}_{1}=v^{\rho_{1}} \zeta_{n}^{-1}\left(2 r_{1}\right)
$$

Then, from Proposition 4.2.22 we know that, there exists a word $p \in\left\langle C_{n}, C_{n-\operatorname{sgn}(n)}\right\rangle$ and an integer $\rho_{2} \in \mathbb{Z}$ such that

$$
\begin{aligned}
\hat{v} & =p p^{-}, \\
\hat{w}_{2} & =\left(p p^{-}\right)^{\rho_{2}} p \zeta_{n}^{-1}\left(2 r_{2}+2\right) C_{0} .
\end{aligned}
$$

Thus

$$
\hat{w}_{1}=\left(p p^{-}\right)^{\rho_{1}} \zeta_{n}^{-1}\left(2 r_{1}\right)
$$

To compute $\mu_{r}\left(\hat{w}_{1}\right)$ and $\eta\left(\hat{w}_{2}^{-1}\right) \hat{w}_{1}^{-1} \hat{w}_{2}$ we use the identities of Propositions 4.2.9, 4.2.21 and 4.2.16. Recall that $\eta \circ \mu_{2 r_{2}+1}=\eta \circ \mu_{r} \circ \eta=\mu_{r}$, (see Equation (4.9)). Considering that $p \in$ $\left\langle C_{n-\operatorname{sngn}(n)}, C_{n}\right\rangle$ it follows, by Propositions 4.2.21 and 4.2.16,

$$
\begin{aligned}
\eta\left(\hat{w}_{2}^{-1}\right) & =\eta\left(C_{0}^{-1} \zeta_{n}\left(2 r_{2}+2\right) p^{-1}\left(p p^{-}\right)^{-\rho_{2}}\right) \\
& =C_{0}^{-1}\left[\zeta_{n}(r+1) \zeta_{n}^{-1}\left(2 r_{1}\right)\right]\left[\zeta_{n}\left(2 r_{1}\right) p^{-1}\left(p p^{-}\right)^{-\rho_{2}} \zeta_{n}^{-1}\left(2 r_{1}\right)\right] \\
& =C_{0}^{-1} \zeta_{n}(r+1) p^{-1}\left(p p^{-}\right)^{-\rho_{2}} \zeta_{n}^{-1}\left(2 r_{1}\right) .
\end{aligned}
$$

Also

$$
\begin{aligned}
\hat{w}_{1}^{-1} \hat{w}_{2} & =\left[\zeta_{n}\left(2 r_{1}\right)\left(p p^{-}\right)^{-\rho_{1}}\right]\left[\left(p p^{-}\right)^{\rho_{2}} p \zeta_{n}^{-1}\left(2 r_{2}+2\right) C_{0}\right] \\
& =\zeta_{n}\left(2 r_{1}\right)\left(p p^{-}\right)^{-\rho_{1}+\rho_{2}} p \zeta_{n}^{-1}\left(2 r_{2}+2\right) C_{0} .
\end{aligned}
$$


Therefore

$$
\begin{aligned}
\eta\left(\hat{w}_{2}^{-1}\right) \hat{w}_{1}^{-1} \hat{w}_{2} & =\left[C_{0}^{-1} \zeta_{n}(r+1) p^{-1}\left(p p^{-}\right)^{-\rho_{2}} \zeta_{n}^{-1}\left(2 r_{1}\right)\right]\left[\zeta_{n}\left(2 r_{1}\right)\left(p p^{-}\right)^{-\rho_{1}+\rho_{2}} p \zeta_{n}^{-1}\left(2 r_{2}+2\right) C_{0}\right] \\
& =\left[C_{0}^{-1} \zeta_{n}(r+1)\right] p^{-1}\left(p p^{-}\right)^{-\rho_{2}-\rho_{1}+\rho_{2}} p\left[\zeta_{n}^{-1}\left(2 r_{2}+2\right) C_{0}\right] \\
& =\left[C_{0}^{-1} \zeta_{n}(r+1)\right]\left(p^{-} p\right)^{-\rho_{1}}\left[\zeta_{n}^{-1}\left(2 r_{2}+2\right) C_{0}\right] .
\end{aligned}
$$

Computing $\mu_{r}\left(\hat{w}_{1}\right)$ gives us

$$
\begin{aligned}
\mu_{r}\left(\hat{w}_{1}\right) & =\mu_{r}\left(\left(p p^{-}\right)^{\rho_{1}} \zeta_{n}^{-1}\left(2 r_{1}\right)\right) \\
& =\mu_{r}\left(p p^{-}\right)^{\rho_{1}} \mu_{r}\left(\zeta_{n}^{-1}\left(2 r_{1}\right)\right) \\
& \left.=\left[C_{0}^{-1} \zeta_{n}(r+1)\left(p^{-} p\right)_{1}^{\rho} \zeta_{n}^{-1}(r+1) C_{0}\right]\left[C_{0}^{-1} \zeta_{n}(r+1) \zeta_{n}^{-1}\left(2 r_{2}+2\right) C_{0}\right)\right] \\
& =C_{0}^{-1} \zeta_{n}(r+1)\left(p^{-} p\right)_{1}^{\rho} \zeta_{n}^{-1}\left(2 r_{2}+2\right) C_{0} .
\end{aligned}
$$

Hence, $\mu_{r}\left(\hat{w}_{1}\right)=\eta\left(\hat{w}_{2}^{-1}\right) \hat{w}_{1}^{-1} \hat{w}_{2}$ if and only if $\left(p^{-} p\right)^{\rho_{1}}=\left(p^{-} p\right)^{-\rho_{1}}$. But $p p^{-}=1$ would imply that

$$
\hat{w}_{2} \mu_{2 r_{2}+1}\left(\hat{w}_{2}\right)=\hat{v}=p p^{-}=1
$$

is the empty word, which is not possible by hypothesis. Thus $\rho_{1}=0$ and

$$
\begin{aligned}
& \hat{w}_{1}=\zeta_{n}^{-1}\left(2 r_{1}\right), \\
& \hat{w}_{2}=\left(p p^{-}\right)^{\rho_{2}} p \zeta_{n}^{-1}\left(2 r_{2}+2\right) C_{0} .
\end{aligned}
$$

and consequently

$$
\begin{aligned}
& w_{1}=u^{-1} \zeta_{n}^{-1}\left(2 r_{1}\right) \eta(u) \\
& w_{2}=u^{-1}\left(p p^{-}\right)^{\rho_{2}} p \zeta_{n}^{-1}\left(2 r_{2}+2\right) C_{0} \mu_{2 r_{2}+1}(u) .
\end{aligned}
$$

For the converse, using the same arguments it is not too difficult to show that for $w_{1}$ and $w_{2}$ of the form above the following equation is satisfied

$$
\mu_{r}\left(w_{1}\right)=\eta\left(w_{2}^{-1}\right) w_{1}^{-1} w_{2} .
$$

\subsection{Classification of based homotopy classes with Nielsen number zero}

From the last section we conclude the following.

Theorem 4.3.1. Let $\phi: K \multimap K$ be a 2-valued split map with Nielsen number zero, let $\Phi: K \rightarrow$ $D_{2}(K)$ be the correspondent map and $\hat{\Phi}: K \rightarrow F_{2}(K)$ its lift with $\hat{\Phi}\left(x_{0}\right)=\left(p_{1}, p_{2}\right)$. Then

$$
\begin{aligned}
\hat{\Phi}_{\#}: \pi_{1}(K) & \longrightarrow F_{2}\left(a_{2}, b_{2}\right) \rtimes \pi_{1}(K) \\
\alpha & \longmapsto \tilde{\alpha}=w_{1} a_{1}^{r_{1}} \\
\beta & \longmapsto \tilde{\beta}=w_{2} a_{1}^{r_{2}} b_{1},
\end{aligned}
$$

where $w_{1}$ and $w_{2} \in H$ as in one of the cases that follows:

i) There are words $u_{1}, u_{2} \in H$ and integers $s_{1}, s_{2}, n_{1}, n_{2}$ such that

$$
\begin{aligned}
& w_{1}=\left[u_{2} \xi_{n_{2}}^{s_{2}}\left(2 r_{2}+2\right) \mu_{2 r_{2}+1}\left(u_{2}^{-1}\right)\right]\left[u_{1} \xi_{n_{1}}^{s_{1}}(r+1) \mu_{r}\left(u_{1}^{-1}\right)\right] \\
& w_{2}=u_{2} \xi_{n_{2}}^{s_{2}}\left(2 r_{2}+2\right) \mu_{2 r_{2}+1}\left(u_{2}^{-1}\right) .
\end{aligned}
$$


ii) There exist an integer $\rho \in \mathbb{Z}$, a non-zero integer $n$, a word $u \in H$ and a word $p \in\left\langle C_{n-\operatorname{sgn}(n)}, C_{n}\right\rangle$ with $p^{-}$a primitive cyclically reduced word such that

$$
\begin{aligned}
& w_{1}=u^{-1} \zeta_{n}^{-1}\left(2 r_{1}\right) \eta(u), \\
& w_{2}=u^{-1}\left(p p^{-}\right)^{\rho} p \zeta_{n}^{-1}\left(2 r_{2}+2\right) C_{0} \mu_{2 r_{2}+1}(u) .
\end{aligned}
$$

Conversely, let $w_{1}$ and $w_{2}$ be either

$$
\begin{aligned}
& w_{1}=\left[u_{2} \xi_{n_{2}}^{s_{2}}\left(2 r_{2}+2\right) \mu_{2 r_{2}+1}\left(u_{2}^{-1}\right)\right]\left[u_{1} \xi_{n_{1}}^{s_{1}}(r+1) \mu_{r}\left(u_{1}^{-1}\right)\right] \\
& w_{2}=u_{2} \xi_{n_{2}}^{s_{2}}\left(2 r_{2}+2\right) \mu_{2 r_{2}+1}\left(u_{2}^{-1}\right),
\end{aligned}
$$

where $u_{1}, u_{2} \in H$ and $s_{1}, s_{2}, n_{1}, n_{2} \in \mathbb{Z}$ or

$$
\begin{aligned}
& w_{1}=u^{-1} \zeta_{n}^{-1}\left(2 r_{1}\right) \eta(u) \\
& w_{2}=u^{-1}\left(p p^{-}\right)^{\rho} p \zeta_{n}^{-1}\left(2 r_{2}+2\right) C_{0} \mu_{2 r_{2}+1}(u),
\end{aligned}
$$

with $\rho, n \in \mathbb{Z}, n \neq 0, u \in H$ and $p \in\left\langle C_{n-\operatorname{sgn}(n)}, C_{n}\right\rangle$ with $p p^{-}$a primitive cyclically reduced. Then

$$
\begin{aligned}
\pi_{1}(K) & \longrightarrow F_{2}\left(a_{2}, b_{2}\right) \rtimes \pi_{1}(K) \\
\alpha & \longmapsto \tilde{\alpha}=w_{1} a_{1}^{r_{1}} \\
\beta & \longmapsto \tilde{\beta}=w_{2} a_{1}^{r_{2}} b_{1},
\end{aligned}
$$

is a homomorphism induced by a 2-valued split map $\phi: K \multimap K$ with Nielsen number zero.

Proof. Let $\hat{\Phi}: K \rightarrow F_{2}(K)$ be a map with Nielsen number zero. Then from Theorem 4.1.8 it follows that there are words $w_{1}$ and $w_{2}$ in the free group $F_{2}$ and integers $r_{1}, r_{2} \in \mathbb{Z}$ such that

$$
\begin{aligned}
\hat{\Phi}_{\#}: \pi_{1}\left(K, p_{0}\right) & \longrightarrow F_{2} \rtimes_{\theta_{1}} \pi_{1}(K) \\
\alpha & \longmapsto \hat{\alpha}=w_{1} a_{1}^{r_{1}} \\
\beta & \longmapsto \hat{\beta}=w_{2} a_{1}^{r_{2}} b_{1},
\end{aligned}
$$

where $w_{1}, w_{2} \in H=\left\langle w \in F_{2}:|w|^{b_{2}}=0\right\rangle$ such that equation 4.4 holds, that is

$$
w_{2}=w_{1} \theta_{1}\left(a_{1}^{r_{1}}\right)\left(w_{2}\right) \theta_{1}\left(a_{1}^{r_{1}+r_{2}} b_{1}\right)\left(w_{1}\right) .
$$

Using the notation defined in (4.7) we know that equation 4.4 is equivalent to equation (4.11)

$$
\mu_{r}\left(w_{1}\right)=\eta\left(w_{2}^{-1}\right) w_{1}^{-1} w_{2},
$$

where $r=2\left(r_{1}+r_{2}\right)+1$. From Corollary 4.2.1.1 this implies that equation 4.12 holds, that is

$$
w_{1}=\left(w_{2} \mu_{2 r_{2}+1}\left(w_{2}\right)\right)^{-1} w_{1} \eta\left(w_{2} \mu_{2 r_{2}+1}\left(w_{2}\right)\right) .
$$

We divide into two cases, either $w_{2} \mu_{2 r_{2}+1}\left(w_{2}\right)=1$ or $w_{2} \mu_{2 r_{2}+1}\left(w_{2}\right)$ is not the empty word.

If $w_{2} \mu_{2 r_{2}+1}\left(w_{2}\right)=1$ then Theorem 4.2.14 implies that there are words $u_{1}, u_{2} \in H$ and integers $s_{1}, s_{2}, n_{1}, n_{2}$ such that

$$
\begin{aligned}
& w_{1}=\left[u_{2} \xi_{n_{2}}^{s_{2}}\left(2 r_{2}+2\right) \mu_{2 r_{2}+1}\left(u_{2}^{-1}\right)\right]\left[u_{1} \xi_{n_{1}}^{s_{1}}(r+1) \mu_{r}\left(u_{1}^{-1}\right)\right] \\
& w_{2}=u_{2} \xi_{n_{2}}^{s_{2}}\left(2 r_{2}+2\right) \mu_{2 r_{2}+1}\left(u_{2}^{-1}\right) .
\end{aligned}
$$

If $w_{2} \mu_{2 r_{2}+1}\left(w_{2}\right) \neq 1$, then Theorem 4.2.23 implies that then there exist integers $\rho \in \mathbb{Z}$, a non zero integer $n$, a word $u \in H$ and a word $p \in\left\langle C_{n-\operatorname{sngn}(n)}, C_{n}\right\rangle$ with $p p^{-}$is a primitive cyclically 
reduced word such that

$$
\begin{aligned}
& w_{1}=u^{-1} \zeta_{n}^{-1}\left(2 r_{1}\right) \eta(u) \\
& w_{2}=u^{-1}\left(p p^{-}\right)^{\rho} p \zeta_{n}^{-1}\left(2 r_{2}+2\right) C_{0} \mu_{2 r_{2}+1}(u) .
\end{aligned}
$$

For the converse note that if $w_{1}$ and $w_{2}$ are one of the cases stated above Theorem 4.2.14 and Theorem 4.2.23 respectively imply that the following equation holds

$$
\mu_{r}\left(w_{1}\right)=\eta\left(w_{2}^{-1}\right) w_{1}^{-1} w_{2} .
$$

Then Theorem 4.1.8 implies that there is 2-valued map $\phi$ with Nielsen number zero that induces exactly the homomorphism above. 


\section{Chapter 5}

\section{Nielsen number of 2-valued non-split maps on the Klein bottle}

In chapter 4 we compute the Nielsen number of 2-valued split maps and give a classification for those with Nielsen number zero. For non-split maps the computations are more complicated. In this chapter we give a formula for the Nielsen number of 2-valued maps that are not split.

For a non-split 2-valued map $\phi: K \multimap K$ with correspondent map $\Phi: K \rightarrow D_{2}(K)$ be the correspondent map, then the induced homomorphism is in the form

$$
\begin{aligned}
\Phi_{\#}: \pi_{1}(K) & \rightarrow B_{2}(K) \\
\alpha & \mapsto \hat{\alpha} \\
\beta & \mapsto \hat{\beta},
\end{aligned}
$$

where $\Phi$ is one of the following types
(A) $\hat{\alpha}$ pure and $\hat{\beta}$ not pure,
(B) $\hat{\alpha}$ not pure and $\hat{\beta}$ pure and
(C) both $\hat{\alpha}$ and $\hat{\beta}$ not pure.

If $\hat{\alpha}$ and $\hat{\beta}$ are pure, then $\Phi_{\#}\left(\pi_{1}(K)\right) \subset P_{2}(K)$, thus by Observation 3.1.3 the map $\phi$ is split. Recall that $\Phi^{-1}\left(P_{2}(K)\right)$, subset of $\pi_{1}(K)$ induces a double covering space $q: \tilde{K} \rightarrow K$ with a unique Deck-transformation $\delta$ (see 3.2.2). We see that there is a map $\hat{\Phi}=\left(\hat{f}_{1}, \hat{f}_{2}\right)$ which is a lifting of $\Phi \circ q$. We can consider a map $\Omega:[K, K]_{0} \rightarrow[\tilde{K}, K]_{0} \times[\tilde{K}, K]_{0}$, that takes a (based) homotopy class of a 2 -valued non-split map $[\phi]$ to a pair $\left(\left[\hat{f}_{1}\right]\left[\hat{f}_{2}\right]\right)$, where $\left(\hat{f}_{1}, \hat{f}_{2}\right)$ is the lifting of $\Phi \circ q$. We are interested in determining the Image of this map and manage to do so for maps of type $(B)$ and $(C)$. Proposition 3.2.5 proves that the homotopy class $\left[\hat{f}_{1}\right]$ does not have the Borsuk-Ulam property and $\hat{f}_{2}=\hat{f}_{1} \circ \delta$. For maps of type $(B)$ and $(C)$ the covering space is the Klein bottle and there is a characterisation for maps that have not the Borsuk-Ulam property in respect to $\delta$. We use this characterisation to describe the image of $\Omega$. Also there is a one-to-one correspondence between maps of type $(B)$ and maps of type $(C)$, using a homeomorphism from $K$ to $K$. The covering space induced by maps of type $(A)$ is the torus. There is no classification (yet) for maps from the torus to the Klein bottle that have not the Borsuk-Ulam property in respect to the involution that does not preserves orientation. Therefore we use that the Nielsen coincidence number $N\left(f_{1}, f_{2}\right)$ is zero to give some necessary conditions for $\hat{\alpha}$ and $\hat{\beta}$. Also we compute the Nielsen number for map of each type in terms of $\hat{\alpha}$ and $\hat{\beta}$. 


\subsection{Maps of type (A)}

Let $\phi: K \multimap K$ be a 2-valued map of type $(A)$ and consider the correspondent map $\Phi: K \rightarrow$ $D_{2}(K)$ with $\Phi\left(x_{0}\right)=\left\{p_{1}, p_{2}\right\}$. Then the fundamental group induced homomorphism is given by

$$
\begin{aligned}
\Phi_{\#}: \pi_{1}(K) & \longrightarrow B_{2}(K) \\
\alpha & \longmapsto \hat{\alpha}=w_{1} a_{1}^{r_{1}} b_{1}^{s_{1}} \\
\beta & \longmapsto \hat{\beta}=w_{2} a_{1}^{r_{2}} b_{1}^{s_{2}} \sigma,
\end{aligned}
$$

where the pure braid $\hat{\alpha}$ and the not pure braid $\hat{\beta}$ are in their normal forms in the presentation of $B_{2}(K)$ given by Theorem 2.2.2, i.e. $w_{1}, w_{2}$ are words in $F_{2}$ and $r_{1}, s_{1}, r_{2}, s_{2} \in \mathbb{Z}$. To better determine $w_{1}$ and $w_{2}$ consider the projection

$$
p r: F_{2}(a, b) \rightarrow F_{2}(a, b) /\left\langle a b a b^{-1}\right\rangle
$$

and let $m_{i}, n_{i} \in \mathbb{Z}$ be the integers such that

$$
\operatorname{pr}\left(w_{i}\right)=a^{m_{i}} b^{n_{i}},
$$

where $i \in\{1,2\}$.

Since $\Phi_{\#}$ is a homomorphism the following equation holds

$$
\hat{\alpha} \hat{\beta} \hat{\alpha}=\hat{\beta} \text {. }
$$

The next lemma explores some of the more direct facts that can be derived from this equation.

Lemma 5.1.1. Let $\hat{\alpha}=w_{1} a_{1}^{r_{1}} b_{1}^{s_{1}}$ and $\hat{\beta}=w_{2} a_{1}^{r_{2}} b_{1}^{s_{2}} \sigma$ with $\operatorname{pr}\left(w_{i}\right)=a^{m_{i}} b^{n_{i}}$, then Equation (5.1) implies $n_{1}=-2 s_{1}$ and

$$
m_{1}=\left((-1)^{s_{1}+s_{2}}-(-1)^{s_{2}}\right) r_{2}-\left(1+(-1)^{s_{1} s_{2}}\right) r_{1} .
$$

Proof. From 2.2.5 it follows that

$$
\hat{\beta} \hat{\alpha}=w a_{1}^{r_{2}+(-1)^{s_{2}} m_{1}+(-1)^{s_{2}+n_{1}} r_{1}} b_{1}^{s_{2}+n_{1}+s_{1}} \sigma,
$$

where $w \in F_{2}$ with $\operatorname{pr}(w)=a_{2}^{m_{2}-(-1)^{s_{2}+m_{2}} m_{1}}$. Hence

$$
\begin{aligned}
\hat{\alpha} \hat{\beta} \hat{\alpha} & =w_{1} a_{1}^{r_{1}} b_{1}^{s_{1}} w a_{1}^{r_{2}+(-1)^{s_{2}} m_{1}+(-1)^{s_{2}+n_{1}} r_{1}} b_{1}^{s_{2}+n_{1}+s_{1}} \sigma, \\
& =w_{1} \theta\left(a_{1}^{r_{1}} b_{1}^{s_{1}}\right)(w) a_{1}^{(-1)^{s_{1}} r_{2}+(-1)^{s_{1}+s_{2}} m_{1}+\left(1+(-1)^{s_{1} s_{2}+n_{1}}\right) r_{1}} b_{1}^{s_{2}+n_{1}+2 s_{1}} \sigma .
\end{aligned}
$$

Now this element in $F_{2} \rtimes_{\theta} \pi_{1}(K)$ is equal to $\hat{\beta}$, hence we compare the components and it follows

$$
\begin{aligned}
w_{2} & =w_{1} \theta\left(a_{1}^{r_{1}} b_{1}^{s_{1}}\right)(w) \\
\text { and } & \\
a_{1}^{r_{2}} b_{1}^{s_{2}} & =a_{1}^{(-1)^{s_{1}} r_{2}+(-1)^{s_{1}+s_{2}} m_{1}+\left(1+(-1)^{s_{1}+s_{2}+n_{1}}\right) r_{1}} b_{1}^{s_{2}+n_{1}+2 s_{1}} .
\end{aligned}
$$

Thus $n_{1}=-2 s_{1}$ is even and

$$
r_{2}=(-1)^{s_{1}} r_{2}+(-1)^{s_{1}+s_{2}} m_{1}+\left(1+(-1)^{s_{1}+s_{2}+n_{1}}\right) r_{1},
$$


hence

$$
\begin{aligned}
m_{1} & \left.=(-1)^{s_{1}+s_{2}}\left(1-(-1)^{s_{1}}\right) r_{2}-\left(1+(-1)^{s_{1}+s_{2}+n_{1}}\right) r_{1}\right) \\
& =\left((-1)^{s_{1}+s_{2}}-(-1)^{s_{2}}\right) r_{2}-\left(1+(-1)^{s_{1} s_{2}}\right) r_{1} .
\end{aligned}
$$

The subgroup $H=\Phi_{\#}^{-1}\left(P_{2}(K)\right)$ of $\pi_{1}(K)$ is the kernel of the map $\tau \circ \Phi_{\#}$. Using the ReidermeisterSchreier method we find a presentation

$$
H=\left\langle\alpha, \beta^{2}: \alpha \beta^{2}=\beta^{2} \alpha\right\rangle,
$$

which is isomorphic to the fundamental group of the torus $\pi_{1}(T)=\langle a, b: a b=b a\rangle$, identifying $\alpha$ with $a$ and $\beta^{2}$ with $b$. Then the finite regular double covering $q$ that corresponds to $H$ is given by

$$
\begin{aligned}
q_{\#}: \pi_{1}\left(T, \tilde{x}_{0}\right) & \longrightarrow \pi_{1}\left(K, x_{0}\right) \\
a & \longmapsto \alpha \\
b & \longmapsto \beta^{2},
\end{aligned}
$$

where $\tilde{x}_{0} \in q^{-1}\left(x_{0}\right)$. The non-trivial Deck-transformation $\delta: T \rightarrow T$ of the covering $q: T \rightarrow K$ is a free involution and admits a lifting to the plane given by

$$
\hat{\delta}(x, y)=\left(1-x, y+\frac{1}{2}\right) .
$$

Since $(\Phi \circ q)_{\#}\left(\pi_{1}(T)\right) \subset P_{2}(K)$ there is a lifting $\Phi_{1}=\left(f_{1}, f_{2}\right): T \rightarrow F_{2}(K)$ of $\Phi \circ q$, that is the following diagram commutes,

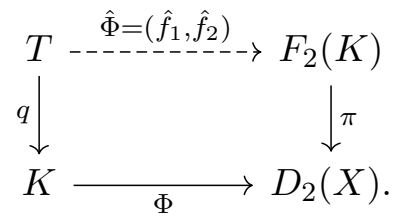

Lemma 5.1.2. Let $\phi: K \multimap K$ be a 2-valued map of type $(A)$, with $\Phi_{\#}$ given as above, and $\hat{\Phi}: T \rightarrow F_{2}(K)$ be a lifting of $\Phi \circ q$ with $\hat{\Phi}\left(\tilde{x}_{0}\right)=\left(p_{1}, p_{2}\right)$, where $\tilde{x}_{0} \in q^{-1}\left(x_{0}\right)$. Then $\hat{\Phi}=\left(\hat{f}_{1}, \hat{f}_{2}\right)$, where $\hat{f}_{1}, \hat{f}_{2}: T \rightarrow K$ are given by

$$
\begin{aligned}
& \left(\hat{f}_{1}\right)_{\#}: \pi_{1}\left(T, \tilde{x}_{0}\right) \longrightarrow \pi_{1}\left(K, p_{1}\right) \\
& a \longmapsto a_{1}^{r_{1}} b_{1}^{s_{1}} \\
& b \longmapsto a_{1}^{r_{2}+(-1)^{s_{2}} m_{2}+(-1)^{n_{2}+s_{2}} r_{2}} b_{1}^{2 s_{2}+n_{2}}, \\
& \left(\hat{f}_{2}\right)_{\#}: \pi_{1}\left(T, \tilde{x}_{0}\right) \longrightarrow \pi_{1}\left(K, p_{2}\right) \\
& a \longmapsto a_{2}^{m_{1}+(-1)^{n_{1}} r_{1}} b_{2}^{-s_{1}} \\
& b \longmapsto a_{2}^{m_{2}+(-1)^{n_{2}} r_{2}+(-1)^{n_{2}+s_{2}} r_{2}} b_{2}^{2 s_{2}+n_{2}} \text {. }
\end{aligned}
$$

Proof. We know that $(\Phi \circ q)_{\#}(a)$ and $(\Phi \circ q)_{\#}(b)$ are pure braids that using the presentation given by Theorem 2.2.2 are given as elements in $F_{2} \rtimes_{\theta_{1}} \pi_{1}(K)$. Consider the isomorphism $\Gamma: F_{2} \rtimes_{\theta_{1}} \pi_{1}(K) \rightarrow$ $P_{2}(K)$ given in Corollary 2.1.3.1 and $p r_{i}: F_{2}(K) \rightarrow K$, the projection to the $i$-th component. Then $\left(p r_{i} \circ \Gamma \circ \Phi \circ q\right)\left(\tilde{x}_{0}\right)=p_{i}$ for $i \in\{1,2\}$. Set $f_{i}=p r_{i} \circ \Gamma \circ \Phi \circ q: T \rightarrow K$, then from the uniqueness of the lifting, $\Phi_{1}$ is equal to $\left(f_{1}, f_{2}\right)$. 
Consider

$$
\begin{aligned}
(\Gamma \circ \Phi \circ q)_{\#}(a) & =(\Gamma \circ \Phi)_{\#}(\alpha) \\
& =(\Gamma \circ)_{\#}\left(w_{1} a_{1}^{r_{1}} b_{1}^{s_{1}}\right) \\
& =w_{1}\left(a_{2} a_{1}\right)^{r_{1}}\left(b_{2} b_{1}\right)^{s_{1}} .
\end{aligned}
$$

Therefore

$$
\begin{aligned}
& \left(f_{1}\right)_{\#}(a)=\operatorname{pr}_{1}\left(w_{1}\left(a_{2} a_{1}\right)^{r_{1}}\left(b_{2} b_{1}\right)^{s_{1}}\right)=a_{1}^{r_{1}} b_{1}^{s_{1}}, \\
& \left(f_{2}\right)_{\#}(a)=\operatorname{pr}_{2}\left(w_{1}\left(a_{2} a_{1}\right)^{r_{1}}\left(b_{2} b_{1}\right)^{s_{1}}\right)=a_{2}^{m_{1}} b_{2}^{n_{1}} a_{2}^{r_{1}} b_{2}^{s_{1}}=a_{2}^{m_{1}+(-1)^{n_{1}} r_{1}} b_{2}^{n_{1}+s_{1}} .
\end{aligned}
$$

From Lemma 5.1.1 we know that $n_{1}=-2 s_{1}$, hence

$$
\left(f_{2}\right)_{\#}(a)=a_{2}^{m_{1}+(-1)^{n_{1}} r_{1}} b_{2}^{-s_{1}} .
$$

Since $(q)_{\#}(b)=\beta^{2}$ the normal form of $(\Phi \circ q)_{\#}(b)$ is more difficult to compute. Using the relations in Theorem 2.2.2 and the identities of Lemma 2.2.4 it follows

$$
\begin{aligned}
(\Gamma \circ \Phi \circ q)_{\#}(b) & =(\Gamma \circ \Phi)_{\#}\left(\beta^{2}\right) \\
& =(\Gamma)_{\#}\left(\hat{\beta}^{2}\right) \\
& =(\Gamma)_{\#}\left(w_{2} a_{1}^{r_{2}} b_{1}^{s_{2}} \sigma\right)^{2} \\
& =(\Gamma)_{\#}\left(w_{2} a_{1}^{r_{2}} b_{1}^{s_{2}} \sigma w_{2} a_{1}^{r_{2}} b_{1}^{s_{2}} \sigma\right) \\
& =(\Gamma)_{\#}\left(w_{2} a_{1}^{r_{2}} b_{1}^{s_{2}}\left(\sigma w_{2} \sigma^{-1}\right) a_{1}^{r_{2}}\left(b_{2}^{-1} a_{2} b_{2} a_{2} b_{1}\right)^{s_{2}} \sigma^{2}\right) \\
& \left.=w_{2}\left(a_{2} a_{1}\right)^{r_{2}}\left(b_{2} b_{1}\right)^{s_{2}}(\Gamma)_{\#}\left(\sigma w_{2} \sigma^{-1}\right)\left(a_{2} a_{1}\right)^{r_{2}}\left(b_{2}^{-1} a_{2} b_{2} a_{2} b_{2} b_{1}\right)^{s_{2}}\left(b_{2}^{-1} a_{2} b_{2} a_{2}\right)\right) .
\end{aligned}
$$

For the word $w_{2}\left(a_{2}, b_{2}\right) \in F_{2}\left(a_{2}, b_{2}\right)$ it holds

$$
\begin{aligned}
(\Gamma)_{\#}\left(\sigma w_{2}\left(a_{2}, b_{2}\right) \sigma^{-1}\right) & =(\Gamma)_{\#}\left(w_{2}\left(b_{2}^{-1} a_{2} b_{2} a_{1}, b_{2}^{-2} a_{2} b_{2} a_{2} b_{1}\right)\right) \\
& =(\Gamma)_{\#}\left(w_{2}\left(b_{2}^{-1} a_{2} b_{2} a_{1}, b_{2}^{-2} a_{2} b_{2} a_{2} b_{1}\right)\right) \\
& \left.=w_{2}\left(b_{2}^{-1} a_{2} b_{2} a_{2} a_{1}, b_{2}^{-2} a_{2} b_{2} a_{2} b_{2} b_{1}\right)\right) .
\end{aligned}
$$

It holds that

$$
\begin{aligned}
\left(p r_{1} \circ \Gamma\right)_{\#}\left(\sigma w_{2}\left(a_{2}, b_{2}\right) \sigma^{-1}\right) & =\left(p r_{1}\right)_{\#}\left(w_{2}\left(b_{2}^{-1} a_{2} b_{2} a_{2} a_{1}, b_{2}^{-2} a_{2} b_{2} a_{2} b_{2} b_{1}\right)\right) \\
& =\left(p r_{1}\right)_{\#}\left(w_{2}\left(a_{1}, b_{1}\right)\right) \\
& =a_{1}^{m_{2}} b_{1}^{n_{2}},
\end{aligned}
$$

i.e.

$$
\left(p r_{1} \circ \Gamma\right)_{\#}\left(\sigma w_{2} \sigma^{-1}\right)=a_{1}^{m_{2}} b_{1}^{n_{2}} .
$$

Hence

$$
\begin{aligned}
\left(f_{1}\right)_{\#}(b) & =\left(p r_{1} \circ \Gamma \circ \Phi \circ q\right)_{\#}\left(\beta^{2}\right) \\
& =\left(p r_{1}\right)_{\#}\left(w_{2}\left(a_{2} a_{1}\right)^{r_{2}}\left(b_{2} b_{1}\right)^{s_{2}}(\Gamma)_{\#}\left(\sigma w_{2} \sigma^{-1}\right)\left(a_{2} a_{1}\right)^{r_{2}}\left(b_{2}^{-1} a_{2} b_{2} a_{2} b_{2} b_{1}\right)^{s_{2}}\left(b_{2}^{-1} a_{2} b_{2} a_{2}\right)\right) \\
& =a_{1}^{r_{2}} b_{1}^{s_{2}} a_{1}^{m_{2}} b_{1}^{n_{2}} a_{1}^{r_{2}} b_{1}^{s_{2}} \\
& =a_{1}^{r_{2}+(-1)^{s_{2}} m_{2}+(-1)^{s_{2}+n_{2}} r_{2}} b_{1}^{2 s_{2}+n_{2}} .
\end{aligned}
$$

To do the same procedure with $p r_{2}$ note that $b_{2}^{-1} a_{2} b_{2} a_{2}$ is in the kernel of $\left(p r_{2}\right)_{\#}: P_{2}(K) \rightarrow$ 
$\pi_{1}(K)$, thus

$$
\begin{aligned}
\left(p r_{2} \circ \Gamma\right)_{\#}\left(\sigma w_{2}\left(a_{2}, b_{2}\right) \sigma^{-1}\right) & =\left(p r_{2}\right)_{\#}\left(w_{2}\left(b_{2}^{-1} a_{2} b_{2} a_{2} a_{1}, b_{2}^{-2} a_{2} b_{2} a_{2} b_{2} b_{1}\right)\right) \\
& =\left(p r_{2}\right)_{\#}\left(w_{2}\left(a_{1}, b_{2}^{-1} b_{2} b_{1}\right)\right) \\
& =1,
\end{aligned}
$$

that is

$$
\left(p r_{2} \circ \Gamma\right)_{\#}\left(\sigma w_{2} \sigma^{-1}\right)=1
$$

Therefore

$$
\begin{aligned}
\left(f_{2}\right)_{\#}(b) & =\left(p r_{2} \circ \Gamma \circ \Phi \circ q\right)_{\#}\left(\beta^{2}\right) \\
& =\left(p r_{2}\right)_{\#}\left(w_{2}\left(a_{2} a_{1}\right)^{r_{2}}\left(b_{2} b_{1}\right)^{s_{2}}(\Gamma)_{\#}\left(\sigma w_{2} \sigma^{-1}\right)\left(a_{2} a_{1}\right)^{r_{2}}\left(b_{2}^{-1} a_{2} b_{2} a_{2} b_{2} b_{1}\right)^{s_{2}}\left(b_{2}^{-1} a_{2} b_{2} a_{2}\right)\right) \\
& =a_{2}^{m_{2}} b_{2}^{n_{2}} a_{2}^{r_{2}} b_{2}^{s_{2}} a_{2}^{r_{2}} b_{2}^{s_{2}} \\
& =a_{2}^{m_{2}+(-1)^{n_{2}} r_{2}+(-1)^{n_{2}+s_{2}} r_{2}} b_{2}^{n_{2}+2 s_{2}} .
\end{aligned}
$$

Lemma 5.1.3. Let $\hat{f}_{1}, \hat{f}_{2}: T \rightarrow F_{2}(K)$ be given as in Lemma 5.1.2 and suppose that the Nielsen coincidence number $N\left(\hat{f}_{1}, \hat{f}_{2}\right)$ is zero. Then, if $s_{1}$ and $n_{2}$ are even, it holds that either $s_{1}=0$ or $m_{2}=(-1)^{s_{2}} m_{2}=-(1+(-1))^{s_{2}} r_{2}$.

Proof. At first consider the base points, obviously $p_{1}$ is not equal to $p_{2}$, but there is an open disc in $K$ such that $p_{1}$ and $p_{2}$ are contained. Then there exists a homeomorphism $\varsigma: K \rightarrow K$ homotopic to the identity that maps $p_{2}$ to $p_{1}$ and fixes every point not contained in the open disc. Then

$$
\begin{aligned}
\left(\varsigma \circ \hat{f}_{2}\right)_{\#}: \pi_{1}\left(T, \tilde{x}_{0}\right) & \longrightarrow \pi_{1}\left(K, p_{1}\right) \\
a & \longmapsto a_{1}^{m_{1}+(-1)^{n_{1}} r_{1}} b_{1}^{-s_{1}} \\
b & \longmapsto a_{1}^{m_{2}+\left((-1)^{n_{2}}+(-1)^{n_{2}+s_{2}}\right) r_{2}} b_{1}^{2 s_{2}+n_{2}} .
\end{aligned}
$$

Since $\varsigma \circ \hat{f}_{2}$ is homotopic to $\hat{f}_{2}$ the $N\left(\hat{f}_{1}, \hat{f}_{2}\right)=N\left(\hat{f}_{1}, \varsigma \circ \hat{f}_{2}\right)$.

Recall that

$$
\begin{aligned}
\left(\hat{f}_{1}\right)_{\#}: \pi_{1}\left(T, \tilde{x}_{0}\right) & \longrightarrow \pi_{1}\left(K, p_{1}\right) \\
a & \longmapsto a_{1}^{r_{1}} b_{1}^{s_{1}} \\
b & \longmapsto a_{1}^{(-1)^{s_{2}} m_{2}+\left(1+(-1)^{n_{2}+s_{2}}\right) r_{2}} b_{1}^{2 s_{2}+n_{2}},
\end{aligned}
$$

Note that $\hat{f}_{1}$ and $\varsigma \circ \hat{f}_{2}$ are of the same type (see notation of Proposition 3.3.2).

By Proposition 3.3.3N $N\left(\hat{f}_{1}, \varsigma \circ \hat{f}_{2}\right)=0$ if both maps are of type $\left.I I\right)$. Also, if both $f_{1}$ and $\varsigma \circ \hat{f}_{2}$ are of type $I$ ), then $s_{1}$ and $n_{2}$ both are even. Hence

$$
\begin{aligned}
N\left(\hat{f}_{1}, \varsigma \circ \hat{f}_{2}\right) & \left.=\frac{1}{2} \mid 0-\left((-1)^{s_{2}}-1\right) m_{2}\right)\left(2 s_{1}\right)\left|+\frac{1}{2}\right| 0-\left(\left(1+(-1)^{s_{2}}\right) m_{2}+\left(1+(-1)^{s_{2}}\right) r_{2}\right)\left(2 s_{1}\right) \mid \\
& \left.=\mid\left(1-(-1)^{s_{2}}\right) m_{2}\right) s_{1}|+|\left(1+(-1)^{s_{2}}\right)\left(m_{2}+2 r_{2}\right) s_{1} \mid .
\end{aligned}
$$

Suppose that $s_{1} \neq 0$, then $(-1)^{s_{2}} m_{2}=m_{2}$ and

$$
\left(1+(-1)^{s_{2}}\right) 2 r_{2}=-\left(1+(-1)^{s_{2}}\right) m_{2}=-2 m_{2} .
$$


Thus if $s_{1} \neq 0$ then

$$
m_{2}=(-1)^{s_{2}} m_{2}=-\left(1+(-1)^{s_{2}}\right) r_{2} .
$$

Therefore, if $\phi$ is a 2-valued map of type $(A)$, by Lemma 5.1.2 $\hat{f}_{1}$ and $\hat{f}_{2}$ are given in a certain way and the last lemma gives some information about $s_{1}, n_{2}, m_{2}$ and $r_{2}$. These conditions are necessary, but not necessarily sufficient.

Proposition 5.1.4. Let $\phi: K \multimap K$ of type $(A)$, i.e.

$$
\begin{aligned}
\Phi_{\#}: \pi_{1}\left(K, x_{0}\right) & \longrightarrow B_{2}(K) \\
\alpha & \longmapsto \hat{\alpha}=w_{1} a_{1}^{r_{1}} b_{1}^{s_{1}} \\
\beta & \longmapsto \hat{\beta}=w_{2} a_{1}^{r_{2}} b_{1}^{s_{2}} \sigma .
\end{aligned}
$$

and let $\hat{\Phi}=\left(\hat{f}_{1}, \hat{f}_{2}\right)$ be a lifting of $\Phi \circ q$ such that $\hat{\Phi}\left(\tilde{x}_{0}\right)=\left(p_{1}, p_{2}\right)$. Then

$$
N(\phi)=N\left(q, \hat{f}_{1}\right)=\left\{\begin{array}{ll}
\left|r_{1}\right|\left|2 s_{2}+n_{2}-2\right| & \text { if } s_{2}, n_{2} \text { even and } r_{1} \neq 0 \\
2\left|2 s_{2}+n_{2}-2\right| & \text { if } s_{2}, n_{2} \text { odd } \\
\left|2 s_{2}+n_{2}-2\right| & \text { otherwise. }
\end{array}\right\}
$$

Proof. From Proposition 3.2.7 it follows that $N(\hat{\phi})=N\left(q, f_{1}\right)=N\left(q, f_{2}\right)$. The covering map $q: T \rightarrow K$ satisfies $q_{\#}(a)=\alpha$ and $q_{\#}(b)=\beta^{2}$. Lemma 5.1.2 implies that $\hat{f}_{1}: T \rightarrow K$ is the homomorphism defined by

$$
\begin{aligned}
\left(f_{1}\right)_{\#}=\pi_{1}\left(T, \tilde{x}_{0}\right) & \longrightarrow \pi_{1}\left(K, x_{0}\right) \\
a & \longmapsto a_{1}^{r_{1}} b_{1}^{s_{1}} \\
b & \longmapsto a_{1}^{(-1)^{s_{2}} m_{2}+\left(1+(-1)^{n_{2}+s_{2}}\right) r_{2}} b_{1}^{2 s_{2}+n_{2}} .
\end{aligned}
$$

To compute the Nielsen coincidence number $N\left(q, \hat{f}_{1}\right)$ we use the formula given in Proposition 3.3.3. Note that according to the classification in Lemma 3.3.2 $q$ is of type $(I)$. The map $\hat{f}_{1}$ can be any type, $I), I I a), I I b)$ or $I I c)$.

I) If $\hat{f}_{1}$ is of type $I$ ), then $s_{1}$ and $2 s_{2}+n_{2}$ and consequently $n_{2}$ are even, hence

$$
\begin{aligned}
N\left(q, \hat{f}_{1}\right)= & \frac{1}{2}\left|\left(r_{1}-1\right)\left(2 s_{2}+n_{2}-2\right)-\left((-1)^{s_{2}} m_{2}+\left(1+(-1)^{s_{2}}\right) r_{2}\right) s_{1}\right| \\
& +\frac{1}{2}\left|\left(r_{1}+1\right)\left(2 s_{2}+n_{2}-2\right)-\left((-1)^{s_{2}} m_{2}+\left(1+(-1)^{s_{2}}\right) r_{2}\right) s_{1}\right| .
\end{aligned}
$$

Since $\left(\hat{f}_{1}, \hat{f}_{2}\right)$ is a map into $F_{2}(K)$ they don't coincide and thus have Nielsen coincidence number $N\left(\hat{f}_{1}, \hat{f}_{2}\right)$ equal to zero. Therefore by Lemma 5.1 .3 it holds that either $s_{1}=0$ or $m_{2}=(-1)^{s_{2}} m_{2}=-(1+(-1))^{s_{2}} r_{2}$. Thus $\left.(-1)^{s_{2}} m_{2}+\left(1+(-1)^{s_{2}}\right) r_{2}\right) s_{1}=0$ and it follows

$$
\begin{aligned}
N\left(q, \hat{f}_{1}\right) & =\frac{1}{2}\left|\left(r_{1}-1\right)\left(2 s_{2}+n_{2}-2\right)\right|+\frac{1}{2}\left|\left(r_{1}+1\right)\left(2 s_{2}+n_{2}-2\right)\right| \\
& =\frac{1}{2}\left|\left(2 s_{2}+n_{2}-2\right)\right|\left(\left|\left(r_{1}-1\right)\right|+\left|\left(r_{1}+1\right)\right|\right) .
\end{aligned}
$$

It is not to difficult to verify that

$$
\left|\left(r_{1}-1\right)\right|+\left|\left(r_{1}+1\right)\right|=\left\{\begin{array}{ll}
2\left|r_{1}\right| & \text { if } r_{1} \neq 0 \\
2 & \text { otherwise. }
\end{array}\right\}
$$


Therefore

$$
N\left(q, \hat{f}_{1}\right)=\left\{\begin{array}{ll}
\left|\left(2 s_{2}+n_{2}-2\right) r_{1}\right| & \text { if } r_{1} \neq 0 \\
\left|\left(2 s_{2}+n_{2}-2\right)\right| & \text { otherwise. }
\end{array}\right\}
$$

II) If $\hat{f}_{1}$ is of type $\left.I I a\right)$ or $\left.I I b\right)$, then either $s_{1}$ or $n_{2}$ is odd, while the other is even and

$$
\left.N\left(q, \hat{f}_{1}\right)=\mid\left(2-2 s_{2}-n_{2}\right)\right) \mid,
$$

III) If $\hat{f}_{1}$ is of type $\left.I I c\right)$, then both $s_{1}$ and $n_{2}$ are odd and

$$
N\left(q, \hat{f}_{1}\right)=2\left|\left(2-2 s_{2}-n_{2}\right)\right| \text {. }
$$

\subsection{Maps of type (B)}

Let $\phi_{2}: K \multimap K$ be a 2-valued map of type $(B)$ and consider the correspondent map $\Phi_{2}: K \rightarrow$ $D_{2}(K)$, with $\Phi_{2}\left(x_{0}\right)=\left\{p_{1}, p_{2}\right\}$. Then the fundamental group induced homomorphism is given by

$$
\begin{aligned}
\left(\Phi_{2}\right)_{\#}: \pi_{1}\left(K, x_{0}\right) & \longrightarrow B_{2}(K) \\
\alpha & \longmapsto \hat{\alpha}=w_{1} a_{1}^{r_{1}} b_{1}^{s_{1}} \sigma \\
\beta & \longmapsto \hat{\beta}=w_{2} a_{1}^{r_{2}} b_{1}^{s_{2}},
\end{aligned}
$$

where the pure braid $\hat{\beta}$ and the not pure braid $\hat{\alpha}$ are in their normal forms in the presentation of $B_{2}(K)$ given by Theorem 2.2 .2 , i.e. $w_{1}, w_{2}$ are words in $F_{2}$ and $r_{1}, s_{1}, r_{2}, s_{2} \in \mathbb{Z}$.

As in for maps of type $(A)$ let $m_{i}, n_{i} \in \mathbb{Z}$ be the integers such that

$$
\operatorname{pr}\left(w_{i}\right)=a^{m_{i}} b^{n_{i}},
$$

where $i \in\{1,2\}$. Of course, since $\left(\Phi_{2}\right)_{\#}$ is a homomorphism it holds that $\hat{\alpha} \hat{\beta} \hat{\alpha}=\hat{\beta}$.

The subgroup $H=\Phi_{\#}^{-1}\left(P_{2}(K)\right)$ of $\pi_{1}\left(K, x_{0}\right)$ is the kernel of the map $\tau \circ \Phi_{\#}$, where $\tau: B_{2}(K) \rightarrow$ $\mathcal{S}_{2}$ is the map as in (2.1). Using the Reidermeister-Schreier method we find a presentation

$$
H=\left\langle\alpha^{2}, \alpha \beta: \alpha^{2}(\alpha \beta) \alpha^{2}=\alpha \beta\right\rangle,
$$

which is isomorphic to the fundamental group of the Klein bottle $\pi_{1}\left(K_{1}\right)=\langle a, b: a b=b a\rangle$, identifying $\alpha^{2}$ with $a$ and $\beta$ with $b$. Then the finite regular double covering $q_{2}$ that corresponds to $H$ is given by

$$
\begin{aligned}
\left(q_{2}\right)_{\#}: \pi_{1}\left(K_{1}, x_{1}\right) & \longrightarrow \pi_{1}\left(K, x_{0}\right) \\
a & \longmapsto \alpha^{2} \\
b & \longmapsto \beta .
\end{aligned}
$$

Let $\delta_{2}$ be the unique Deck-transformation of the covering space. Since $\left(\Phi_{2} \circ q_{2}\right)_{\#}\left(K_{1}\right) \subseteq P_{2}(K)$, there is a unique lifting $\hat{\Phi}_{2}=\left(\hat{f}_{1}, \hat{f}_{2}\right): K_{1} \rightarrow F_{2}(K)$ of $\Phi_{2} \circ q_{2}$ with $\hat{\Phi}_{2}\left(x_{1}\right)=\left(p_{1}, p_{2}\right)$, where $x_{1} \in q_{2}^{-1}\left(x_{0}\right)$. Hence the following diagram commutes

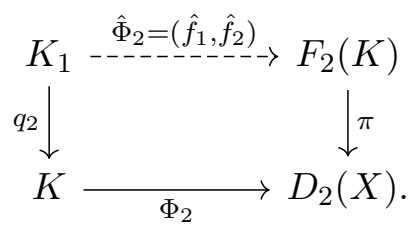


As for maps of type $(A)$ we can compute the (based) homotopy classes of $\hat{f}_{1}$ and $\hat{f}_{2}$, which is done in the next Lemma. 
Lemma 5.2.1. Let $\phi_{2}: K \multimap K$ be a 2-valued map of type $(B)$, with $\hat{\Phi}_{2}=\left(\hat{f}_{1}, \hat{f}_{2}\right)$ lifting of $\Phi_{2} \circ q_{2}$, with $\Phi_{1}\left(x_{1}\right)=\left(p_{1}, p_{2}\right)$, where $x_{1} \in q^{-1}\left(x_{0}\right)$. Then $\hat{f}_{1}, \hat{f}_{2}: K_{1} \rightarrow K$ are given by

$$
\begin{aligned}
\left(\hat{f}_{1}\right)_{\#}: \pi_{1}\left(K_{1}, x_{1}\right) & \longrightarrow \pi_{1}\left(K, p_{1}\right) \\
a & \longmapsto a_{1}^{\left(1+(-1)^{n_{1}+s_{1}}\right) r_{1}+(-1)^{s_{1}} m_{1}} b_{1}^{2 s_{1}+n_{1}} \\
b & \longmapsto a_{1}^{r_{2}} b_{1}^{s_{2}} \\
\left(\hat{f}_{2}\right)_{\#}: \pi_{1}\left(K_{1}, x_{1}\right) & \longrightarrow \pi_{1}\left(K, p_{2}\right) \\
a & \longmapsto a_{2}^{\left((-1)^{n_{1}}+(-1)^{n_{1}+s_{1}}\right) r_{1}+m_{1}} b_{2}^{2 s_{1}+n_{1}} \\
b & \longmapsto a_{2}^{m_{2}+(-1)^{n_{2}} r_{2}} b_{2}^{n_{2}+s_{2}} .
\end{aligned}
$$

Proof. As in proof of Proposition 5.1.2 consider the isomorphism $\Gamma: F_{2} \rtimes_{\theta_{1}} \pi_{1}(K) \rightarrow P_{2}(K)$ given in Corollary 2.1.3.1 and $p_{i}: F_{2}(K) \rightarrow K$, the projection to the $i$-th component. Then $\left(p r_{i} \circ \Gamma \circ \Phi \circ q_{2}\right)\left(\tilde{x}_{0}\right)=p_{i}$ for $i \in\{1,2\}$. Set $f_{i}=p r_{i} \circ \Gamma \circ \Phi \circ q_{2}: K_{1} \rightarrow K$, then from the uniqueness of the lifting, $\Phi_{1}$ is equal to $\left(f_{1}, f_{2}\right)$.

Consider

$$
\begin{aligned}
\left(\Gamma \circ \Phi \circ q_{2}\right)_{\#}(b) & =(\Gamma \circ \Phi)_{\#}(\beta) \\
& =(\Gamma \circ)_{\#}\left(w_{2} a_{1}^{r_{2}} b_{1}^{s_{2}}\right) \\
& =w_{2}\left(a_{2} a_{1}\right)^{r_{2}}\left(b_{2} b_{1}\right)^{s_{2}} .
\end{aligned}
$$

Therefore

$$
\begin{aligned}
& \left(f_{1}\right)_{\#}(b)=p r_{1}\left(w_{2}\left(a_{2} a_{1}\right)^{r_{2}}\left(b_{2} b_{1}\right)^{s_{2}}\right)=a_{1}^{r_{2}} b_{1}^{s_{2}}, \\
& \left(f_{2}\right)_{\#}(b)=\operatorname{pr}_{2}\left(w_{2}\left(a_{2} a_{1}\right)^{r_{2}}\left(b_{2} b_{1}\right)^{s_{2}}\right)=a_{2}^{m_{2}} b_{2}^{n_{2}} a_{2}^{r_{2}} b_{2}^{s_{2}}=a_{2}^{m_{2}+(-1)^{n_{2}} r_{2}} b_{2}^{n_{2}+s_{2}} .
\end{aligned}
$$

For the next step we use the relations in Theorem 2.2.2 and the identities of Lemma 2.2.4. It follows

$$
\begin{aligned}
(\Gamma \circ \Phi \circ q)_{\#}(a) & =(\Gamma \circ \Phi)_{\#}\left(\alpha^{2}\right) \\
& =(\Gamma)_{\#}\left(\hat{\alpha}^{2}\right) \\
& =(\Gamma)_{\#}\left(w_{1} a_{1}^{r_{1}} b_{1}^{s_{1}} \sigma\right)^{2} \\
& =(\Gamma)_{\#}\left(w_{1} a_{1}^{r_{1}} b_{1}^{s_{1}} \sigma w_{1} a_{1}^{r_{1}} b_{1}^{s_{1}} \sigma\right) \\
& =(\Gamma)_{\#}\left(w_{1} a_{1}^{r_{1}} b_{1}^{s_{1}}\left(\sigma w_{1} \sigma^{-1}\right) a_{1}^{r_{1}}\left(b_{2}^{-1} a_{2} b_{2} a_{2} b_{1}\right)^{s_{1}} \sigma^{2}\right) \\
& \left.=w_{1}\left(a_{2} a_{1}\right)^{r_{1}}\left(b_{2} b_{1}\right)^{s_{1}}(\Gamma)_{\#}\left(\sigma w_{1} \sigma^{-1}\right)\left(a_{2} a_{1}\right)^{r_{1}}\left(b_{2}^{-1} a_{2} b_{2} a_{2} b_{2} b_{1}\right)^{s_{1}}\left(b_{2}^{-1} a_{2} b_{2} a_{2}\right)\right) .
\end{aligned}
$$

As in Equations (5.4) and (5.5) it holds

$$
\begin{aligned}
& \left(p r_{1} \circ \Gamma\right)_{\#}\left(\sigma w_{1} \sigma^{-1}\right)=a_{1}^{m_{1}} b_{1}^{n_{1}} . \\
& \left(p r_{2} \circ \Gamma\right)_{\#}\left(\sigma w_{1} \sigma^{-1}\right)=1 .
\end{aligned}
$$

Hence

$$
\begin{aligned}
\left(f_{1}\right)_{\#}(a) & =\left(p r_{1} \circ \Gamma \circ \Phi \circ q\right)_{\#}\left(\alpha^{2}\right) \\
& =\left(p r_{1}\right)_{\#}\left(w_{1}\left(a_{2} a_{1}\right)^{r_{1}}\left(b_{2} b_{1}\right)^{s_{1}}(\Gamma)_{\#}\left(\sigma w_{1} \sigma^{-1}\right)\left(a_{2} a_{1}\right)^{r_{1}}\left(b_{2}^{-1} a_{2} b_{2} a_{2} b_{2} b_{1}\right)^{s_{1}}\left(b_{2}^{-1} a_{2} b_{2} a_{2}\right)\right) \\
& =a_{1}^{r_{1}} b_{1}^{s_{1}} a_{1}^{m_{1}} b_{1}^{n_{1}} a_{1}^{r_{1}} b_{1}^{s_{1}} \\
& =a_{1}^{r_{1}+(-1)^{s_{1}} m_{2}+(-1)^{s_{1}+n_{1}} r_{1}} b_{1}^{2 s_{1}+n_{1}} .
\end{aligned}
$$


Also

$$
\begin{aligned}
\left(f_{2}\right)_{\#}(a) & =\left(p r_{2} \circ \Gamma \circ \Phi \circ q\right)_{\#}\left(\alpha^{2}\right) \\
& =\left(p r_{2}\right)_{\#}\left(w_{1}\left(a_{2} a_{1}\right)^{r_{1}}\left(b_{2} b_{1}\right)^{s_{1}}(\Gamma)_{\#}\left(\sigma w_{1} \sigma^{-1}\right)\left(a_{2} a_{1}\right)^{r_{1}}\left(b_{2}^{-1} a_{2} b_{2} a_{2} b_{2} b_{1}\right)^{s_{1}}\left(b_{2}^{-1} a_{2} b_{2} a_{2}\right)\right) \\
& =a_{2}^{m_{2}} b_{2}^{n_{2}} a_{2}^{r_{1}} b_{2}^{s_{1}} a_{2}^{r_{1}} b_{2}^{s_{1}} \\
& =a_{2}^{m_{1}+(-1)^{n_{1}} r_{2}+(-1)^{n_{1}+s_{1}} r_{1}} b_{2}^{n_{1}+2 s_{1}} .
\end{aligned}
$$

From Proposition 3.2.5 we know that $\left[f_{1}\right]$ does not have the Borsuk-Ulam Property in respect to $\delta_{2}$. The following proposition is a classification by D. Gonçalves, J. Guaschi and V. C. Laass of all homotopy classes in $[K, K]$ that do not have the Borsuk-Ulam property in respect to the free involution $\delta_{2}$ that lifts to a homeomorphism $\hat{\delta}_{2}: \mathbb{R}^{2} \rightarrow \mathbb{R}^{2}$, where $\hat{\delta}_{2}(x, y)=\left(x+\frac{1}{2}, y\right)$.

Proposition 5.2.2. [GGL19, Propositions 38, 39, 35 and 7] A based homotopy class $[f] \in[K, K]_{0}$ does not have the Borsuk-Ulam Property in respect to $\delta_{2}$ if and only if $[f]$ is of type $I$ ) of Lemma 3.2.2, i.e

$$
\begin{aligned}
f_{\#}: \pi_{1}(K) & \longrightarrow \pi_{1}(K) \\
\alpha & \longmapsto \alpha^{r} \\
\beta & \longmapsto \alpha^{s} \beta^{2 t+1},
\end{aligned}
$$

where $r, s, t \in \mathbb{Z}$.

Lemma 5.2.3. Let $\phi_{2}: K \multimap K$ be a 2-valued map of type $(B)$ with $\hat{\Phi}_{2}=\left(\hat{f}_{1}, \hat{f}_{2}\right): K_{1} \rightarrow F_{2}(K)$ lifting of $\Phi_{2} \circ q_{2}$. Then $\hat{f}_{2}=\hat{f}_{1} \circ \delta_{2}$ and $\hat{f}_{1}: K_{1} \rightarrow K$ is given by

$$
\begin{aligned}
\left(\hat{f}_{1}\right)_{\#}: \pi_{1}\left(K_{1}, x_{1}\right) & \longrightarrow \pi_{1}\left(K, p_{1}\right) \\
a & \longmapsto a_{1}^{\left(1+(-1)^{s_{1}}\right) r_{1}+(-1)^{s_{1} m_{1}}} \\
b & \longmapsto a_{1}^{r_{2}} b_{1}^{s_{2}},
\end{aligned}
$$

where $s_{2}$ is odd.

Proof. Lemma 5.2.1 implies that $\hat{f}_{1}: K_{1} \rightarrow K$ is given by

$$
\begin{aligned}
\left(\hat{f}_{1}\right)_{\#}: \pi_{1}\left(K_{1}, x_{1}\right) & \longrightarrow \pi_{1}\left(K, p_{1}\right) \\
a & \longmapsto a_{1}^{\left(1+(-1)^{n_{1}+s_{1}}\right) r_{1}+(-1)^{s_{1}} m_{1}} b_{1}^{2 s_{1}+n_{1}} \\
b & \longmapsto a_{1}^{r_{2}} b_{1}^{s_{2}}
\end{aligned}
$$

From Proposition 3.2.5 it follows that $\hat{f}_{2}=\hat{f}_{1} \circ \delta_{2}$ and $\left[f_{1}\right]$ does not have the Borsuk-Ulam Property in respect to $\delta_{2}$. Hence Proposition 5.2.2 implies that $f_{1}$ is of type $I$ ) of Proposition 3.2.2. Therefore $s_{2}$ is odd and $n_{1}=-2 s_{1}$ is even.

Consider the map $\Omega_{2}$, that takes $\left[\Phi_{2}\right] \in\left[K, D_{2}(K)\right]_{0}$, a homotopy class of a map, where the correspondent map $\phi_{2}$ is of type $(B)$ to the pair $\left(\left[\hat{g}_{1}\right],\left[\hat{g}_{2}\right]\right) \in\left[K_{1}, K\right]_{0} \times\left[K_{1}, K\right]_{0}$ of classes of functions determined by the lifting $\hat{\Phi}_{2}=\left(\hat{g}_{1}, \hat{g}_{2}\right)$ of $\Phi_{2} \circ q_{2}: K_{1} \rightarrow D_{2}(K)$.

Proposition 5.2.4. The Image of $\Omega_{2}$ is set of pairs of classes $\left(\left[\hat{g}_{1}\right],\left[\hat{g}_{2}\right]\right)$, where $\hat{g}_{1}$ is of type $I$ ) of Lemma 3.2.2. Additionally $\hat{g}_{2} \in\left[\hat{g}_{1} \circ \delta_{2}\right]$.

Proof. Let $\hat{g}_{1}, \hat{g}_{2}: K_{1} \rightarrow K$ be as above then, by Proposition 5.2.2, [ $\left.\hat{g}_{1}\right]$ does not have the BorsukUlam Property in respect to $\delta_{2}$. Therefore we know from Proposition 3.2.5 that there is a map 
$\hat{f}_{1} \in\left[\hat{g}_{1}\right]$ and a map $\Phi_{2}: K \rightarrow D_{2}(K)$ such that $\left(\hat{f}_{1}, \hat{f}_{1} \circ \delta_{2}\right): \tilde{X} \rightarrow F_{2}(K)$ is a lifting of $\hat{\Phi} \circ q_{2}$. By construction it holds that $\Phi_{2}$ is of type $(B)$. Since $\hat{g}_{2} \in\left[\hat{g}_{1} \circ \delta_{2}\right]$ It holds that

$$
\left[\hat{g}_{2}\right]=\left[\hat{g}_{1} \circ \delta_{2}\right]=\left[\hat{f}_{1} \circ \delta_{2}\right] .
$$

Hence $\left(\left[g_{1}\right],\left[g_{2}\right]\right) \in \operatorname{Im}\left(\Omega_{2}\right)$.

That the image of $\Omega_{2}$ is as above follows from Proposition 5.2.3.

In the next proposition we compute the Nielsen number of a map of type $(B)$.

Proposition 5.2.5. Let $\phi_{2}: K \multimap K$ of type $(B)$, i.e.

$$
\begin{aligned}
\left(\Phi_{2}\right)_{\#}: \pi_{1}(K) & \longrightarrow B_{2}(K) \\
\alpha & \longmapsto \hat{\alpha}=w_{1} a_{1}^{r_{1}} b_{1}^{s_{1}} \sigma \\
\beta & \longmapsto \hat{\beta}=w_{2} a_{1}^{r_{2}} b_{1}^{s_{2}},
\end{aligned}
$$

with $\hat{\alpha} \hat{\beta} \hat{\alpha}=\hat{\beta}$. Then

$$
N\left(\phi_{2}\right)=\left|1-s_{2}\right| \max \left\{\left|\left(1+(-1)^{s_{1}}\right) r_{1}+(-1)^{s_{1}} m_{1}\right|, 2\right\} .
$$

Proof. From Proposition 3.2.7 it follows that $N\left(\phi_{2}\right)=N\left(\hat{f}_{1}, q_{2}\right)=N\left(\hat{f}_{2}, q_{2}\right)$. The covering map $q_{2}: K_{1} \rightarrow K$ is given by the homomorphism

$$
\begin{aligned}
\left(q_{2}\right)_{\#}: \pi_{1}\left(K_{1}, x_{1}\right) & \longrightarrow \pi_{1}\left(K, x_{0}\right) \\
a & \longrightarrow \alpha^{2} \\
b & \longrightarrow \beta .
\end{aligned}
$$

Lemma 5.2.3 implies that $\hat{f}_{1}: K_{1} \rightarrow K$ is given by the homomorphism

$$
\begin{aligned}
\left(\hat{f}_{1}\right)_{\#}: \pi_{1}\left(K_{1}, x_{1}\right) & \longrightarrow \pi_{1}\left(K, p_{1}\right) \\
a & \longmapsto a_{1}^{\left(1+(-1)^{s_{1}}\right) r_{1}+(-1)^{s_{1}} m_{1}} \\
b & \longmapsto a_{1}^{r_{2}} b_{1}^{s_{2}},
\end{aligned}
$$

where $s_{2}$ is odd. By Proposition 3.3.1 the Nielsen coincidence number is given by

$$
N\left(f_{1}, q_{2}\right)=\left|1-s_{2}\right| \max \left\{\left|\left(1+(-1)^{s_{1}}\right) r_{1}+(-1)^{s_{1}} m_{1}\right|,|2|\right\} .
$$

\subsection{Maps of type (C)}

Let $\phi_{3}: K \multimap K$ be a 2-valued map of type $(C)$ and consider the correspondent map $\Phi_{3}: K \rightarrow$ $D_{2}(K)$. Then the fundamental group induced homomorphism is given by

$$
\begin{aligned}
\left(\Phi_{3}\right)_{\#}: \pi_{1}(K) & \longrightarrow B_{2}(K) \\
\alpha & \longmapsto \hat{\alpha}=\tilde{w}_{1} a_{1}^{\tilde{r}_{1}} b_{1}^{\tilde{s}_{1}} \sigma \\
\beta & \longmapsto \hat{\beta}=\tilde{w}_{2} a_{1}^{\tilde{r}_{2}} b_{1}^{\tilde{s}_{2}} \sigma
\end{aligned}
$$

Here both $\hat{\alpha}$ and $\hat{\beta}$ are not pure braids and it holds $\hat{\alpha} \hat{\beta} \hat{\alpha}=\hat{\beta}$

Again we set $\tilde{m}_{i}, \tilde{n}_{i} \in \mathbb{Z}$ as the integers such that, for $i \in\{1,2\}$,

$$
\operatorname{pr}\left(\tilde{w}_{i}\right)=a^{\tilde{m}_{i}} b^{\tilde{n}_{i}} .
$$


The subgroup $H_{3}=\left(\Phi_{3}\right)_{\#}^{-1}\left(P_{2}(K)\right)$ of $\pi_{1}(K)$ is the kernel of the map $\tau \circ \Phi_{\#}$ and using the Reidermeister-Schreier method we find a presentation

$$
H_{3}=\left\langle\alpha^{2}, \beta: \alpha^{2} \beta \alpha^{2}=\beta\right\rangle,
$$

which is isomorphic to the fundamental group of the Klein bottle $\pi_{1}\left(K_{2}\right)=\langle\tilde{a}, \tilde{b}: \tilde{a} \tilde{b} \tilde{a}=\tilde{b}\rangle$, identifying $\tilde{\alpha}^{2}$ with $\tilde{a}$ and $\tilde{\alpha} \tilde{\beta}$ with $\tilde{b}$. Then the finite regular double covering $q_{3}$ that corresponds to $H_{3}$ is given by the homomorphism

$$
\begin{aligned}
\left(q_{3}\right)_{\#}: \pi_{1}\left(K_{2}, x_{2}\right) & , \longrightarrow \pi_{1}\left(K, x_{0}\right) \\
\tilde{a} & \longmapsto \alpha^{2} \\
\tilde{b} & \longmapsto \alpha \beta .
\end{aligned}
$$

It holds that $\Phi_{3} \circ q_{3}: K_{2} \rightarrow F_{2}(K)$ lifts to a map $\hat{\Phi}_{3}=\left(\hat{g}_{1}, \hat{g}_{2}\right): K_{2} \rightarrow F_{2}(K)$. Consider the map $\Psi: K \rightarrow K$ that induces a homomorphism

$$
\begin{aligned}
\Psi_{\#}: \pi_{1}\left(K, x_{0}\right) & \rightarrow \pi_{1}\left(K, x_{0}\right) \\
\alpha & \mapsto \alpha \\
\beta & \mapsto \alpha \beta .
\end{aligned}
$$

Then there is a lifting $\hat{\Psi}: K_{1} \rightarrow K_{2}$ of $\Psi \circ q_{2}$,

$$
\begin{aligned}
\hat{\Psi}_{\#}: \pi_{1}\left(K_{1}, x_{1}\right) & \rightarrow \pi_{1}\left(K_{2}, x_{2}\right) \\
a & \mapsto \tilde{a} \\
b & \mapsto \tilde{b} .
\end{aligned}
$$

Note that $\Phi_{3} \circ \Psi: K \rightarrow D_{2}(K)$ is a map of type $(B)$ and by Lemma 2.2 .5

$$
\begin{aligned}
\left(\Phi_{3} \circ \Psi\right)_{\#}: \pi_{1}(K) & \longrightarrow B_{2}(K) \\
\alpha & \longmapsto \hat{\alpha}=\tilde{w}_{1} a_{1}^{\tilde{r}_{1}} b_{1}^{\tilde{s}_{1}} \sigma \\
\beta & \longmapsto \tilde{w} a_{1}^{r_{1}+(-1)^{\tilde{s}_{1}} \tilde{m}_{2}+(-1)^{\tilde{s}_{1}+\tilde{n}_{1}} \tilde{r}_{2}} b_{1}^{\tilde{n}_{1}+\tilde{s}_{1}+\tilde{s}_{2}},
\end{aligned}
$$

where $w \in F_{2}$ such that $\operatorname{pr}(\tilde{w})=a_{2}^{\tilde{m}_{1}-(-1)^{\tilde{s}_{1}+\tilde{m}_{1}} \tilde{m}_{2}} b_{2}^{\tilde{m}_{1}}$. Set $\Phi_{2}=\Phi_{3} \circ \Psi$ and suppose that $\hat{\Phi}_{2}=$ $\left(\hat{f}_{1}, \hat{f}_{2}\right): K \rightarrow F_{2}(K)$ is the lifting of $\Phi_{2}$ and we haver the folowing commutative diagram.

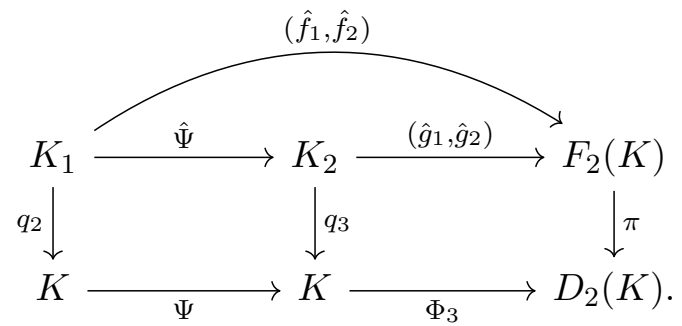

Proposition 5.3.1. Let $\phi_{3}: K \multimap K$ be a 2-valued map of type $(C)$. Then the lifting $\hat{\Phi}_{3}$ of $\Phi_{3} \circ q_{3}$ is equal to $\left(\hat{g}_{1}, \hat{g}_{1} \circ \delta_{3}\right)$, where $\hat{g}_{1}: K \rightarrow K$ is given by

$$
\begin{aligned}
\left(\hat{g}_{1}\right)_{\#}: \pi_{1}\left(K_{2}, x_{2}\right) & \longrightarrow \pi_{1}\left(K, p_{1}\right) \\
\tilde{a} & \longmapsto a_{1}^{\left(1+(-1)^{\tilde{s}_{1}}\right) \tilde{r}_{1}+(-1)^{\tilde{s}_{1}} \tilde{m}_{1}} \\
\tilde{b} & \longmapsto a_{1}^{\tilde{r}_{1}+(-1)^{\tilde{s}_{1}} \tilde{m}_{2}+(-1)^{\tilde{s}_{1}+\tilde{n}_{1}} \tilde{r}_{2}} b_{1}^{\tilde{n}_{1}+\tilde{s}_{1}+\tilde{s}_{2}},
\end{aligned}
$$

where $s_{2}$ is odd. 
Proof. Let $\phi_{3}: K \multimap K$ be a 2-valued map of type $(C)$ with $\hat{\Phi}_{3}=\left(\hat{g}_{1}, \hat{g}_{2}\right): K_{2} \rightarrow F_{2}(K)$ lifting of $\Phi_{3} \circ q_{3}$. Consider the map $\Phi_{2}=\Psi \circ \Phi_{3}$ and $\hat{\Phi}_{2}=\left(\hat{f}_{1}, \hat{f}_{2}\right)$ lifting of $\Phi_{2} \circ q_{2}$. Then

$$
\left(\hat{g}_{1}, \hat{g}_{2}\right) \circ \hat{\Psi}=\left(\hat{f}_{1}, \hat{f}_{2}\right)
$$

Lemma 5.2.3 implies that $\hat{f}_{2}=\hat{f}_{1} \circ \delta_{2}$ and $\hat{f}_{1}: K_{1} \rightarrow K$ is given by

$$
\begin{aligned}
& \left(\hat{f}_{1}\right)_{\#}: \pi_{1}\left(K_{1}, x_{1}\right) \longrightarrow \pi_{1}\left(K, p_{1}\right)
\end{aligned}
$$

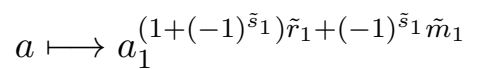

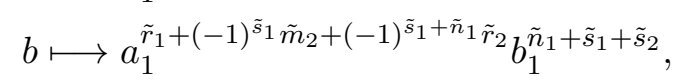

where $\tilde{n}_{1}+\tilde{s}_{1}+\tilde{s}_{2}$ is odd. From $\hat{g}_{1}=\hat{f}_{1} \circ \hat{\Psi}^{-1}$ it directly follows the form of $\hat{g}_{1}$. Now it holds $\delta_{2} \circ \hat{\Psi}=\hat{\Psi} \circ \delta_{3}$, hence

$$
\hat{g}_{2}=\hat{f}_{2} \circ \hat{\Psi}^{-1}=\hat{f}_{1} \circ \delta_{2} \circ \hat{\Psi}^{-1}=\hat{f}_{1} \circ \hat{\Psi}^{-1} \circ \delta_{3}=\hat{g}_{1} \circ \delta_{3} .
$$

Consider the map $\Omega_{3}$ that takes a based homotopy class $\left[\hat{\Phi}_{3}\right] \in\left[K, D_{2}(K)\right]_{0}$ of a map of type $(C)$ to the pair $\left(\left[g_{1}\right],\left[g_{2}\right]\right) \in\left[K_{2}, K\right]_{0} \times\left[K_{2}, K\right]_{0}$ of classes of maps determined by the lifting $\hat{\Phi}_{3}=\left(\hat{g}_{1}, \hat{g}_{2}\right)$ of $\Phi_{3}: K \rightarrow D_{2}(K)$.

Proposition 5.3.2. It holds that the image of $\Omega_{3}$ is equal to the set of pairs of classes $\left(\left[\hat{g}_{1}\right],\left[\hat{g}_{2}\right]\right)$, where $g_{1}$ is of type I) of Lemma 3.2.2. Additionally $g_{2} \in\left[g_{1} \circ \delta_{3}\right]$.

Proof. Let $\hat{g}_{1}, \hat{g}_{2}: K_{2} \rightarrow K$ be as above then composing with $\hat{\Psi}$ gives us a map of type $I$ ) By Proposition 5.2.2, $\left[\hat{g}_{1} \circ \hat{\Psi}\right]$ does not have the Borsuk-Ulam Property in respect to $\delta_{2}$. Therefore we know from Proposition 3.2.5 that there is a map $\hat{f}_{1} \in\left[\hat{g}_{1} \circ \hat{\Psi}\right]$ and a map $\Phi_{2}: K \rightarrow D_{2}(K)$ of type $I I)$ such that $\left(\hat{f}_{1}, \hat{f}_{1} \circ \delta_{2}\right): K_{1} \rightarrow F_{2}(K)$ is a lifting of $\Phi_{2} \circ q_{2}$.

Consider $\Phi_{3}=\Phi_{2} \circ \Psi^{-1}$, which is a map of type $(C)$. Then

$$
\hat{f}_{1} \circ \delta_{2} \circ \hat{\Psi}^{-1}=\hat{f}_{1} \circ \hat{\Psi}^{-1} \circ \delta_{3}=g_{1} \circ \delta_{3},
$$

hence $\left(\hat{g}_{1}, \hat{g}_{1} \circ \delta_{3}\right)$ is a lifting of $\Phi_{3} \circ q_{3}$. Hence $\left(\left[\hat{g}_{1}\right],\left[\hat{g}_{2}\right]\right) \in \operatorname{Im}(\Omega)$.

From Proposition 5.3.1 it follows that every map $\Phi_{3}$ of type $(C)$ has a lifting $\left(\hat{g}_{1}, \hat{g}_{1} \circ \delta_{3}\right)$ that behaves in the wished way.

Proposition 5.3.3. Let $\phi_{3}: K \multimap K$ of type $(C)$ with lifting $\hat{\Phi}_{3}=\left(\hat{f}_{1}, \hat{f}_{2}\right)$ of $\Phi_{3} \circ q_{3}$. Then

$$
N\left(\phi_{3}\right)=\left|1-\tilde{s}_{2}\right| \max \left\{\left|\left(1+(-1)^{\tilde{s}_{1}}\right) \tilde{r}_{1}+(-1)^{\tilde{s}_{1}} \tilde{m}_{1}\right|,|2|\right\}
$$

Proof. From Proposition 3.2.7 it follows that $N(\phi)=N\left(f_{1}, q_{2}\right)=N\left(f_{2}, q_{2}\right)$. The covering map $q_{3}: K_{2} \rightarrow K$ satisfies $\left(q_{3}\right)_{\#}(a)=\alpha^{2}$ and $\left(q_{2}\right)_{\#}(b)=\alpha \beta$.

Proposition 5.3.1 implies that $\hat{g}_{1}: K_{2} \rightarrow K$ is given by the homomorphism

$$
\begin{aligned}
\left(\hat{g}_{1}\right)_{\#}: \pi_{1}\left(K_{2}, x_{2}\right) & \longrightarrow \pi_{1}\left(K, x_{0}\right) \\
a & \longmapsto a_{1}^{\left(1+(-1)^{\tilde{s}_{1}}\right) \tilde{r}_{1}+(-1)^{\tilde{s}_{1}} \tilde{m}_{1}} \\
b & \longmapsto a_{1}^{\tilde{r}_{2}} b_{1}^{\tilde{s}_{2}}
\end{aligned}
$$

where $\tilde{s}_{2}$ is odd. By Proposition 3.3.1 the Nielsen coincidence number is given by

$$
N\left(f_{1}, q_{2}\right)=\left|1-\tilde{s}_{2}\right| \max \left\{\left|\left(1+(-1)^{\tilde{s}_{1}}\right) \tilde{r}_{1}+(-1)^{\tilde{s}_{1}} \tilde{m}_{1}\right|,|2|\right\} .
$$




\section{Bibliography}

[BCES19] Robert F Brown, Michael Crabb, Adam Ericksen e Matthew Stimpson. The 2-sphere is wecken for n-valued maps. Journal of Fixed Point Theory and Applications, 21(2):55, 2019. 1

[Ber97] Claude Berge. Topological Spaces: including a treatment of multi-valued functions, vector spaces, and convexity. Courier Corporation, 1997. 15

[BFGJ05] Robert F Brown, Massimo Furi, Lech Górniewicz e Boju Jiang. Handbook of topological fixed point theory. Springer, 2005. 19

[BG18] Robert F Brown e Daciberg Lima Goncalves. On the topology of n-valued maps. Advances in Fixed Point Theory, 8(2):205-220, 2018. 16

[Bro20] LEJ Brouwer. Über die minimalzahl der fixpunkte bei den klassen von eindeutigen stetigen transformationen der ringlfächen. Mathematische Annalen, 82(1):94-96, 1920. 1

[DMeP15] Carolina De Miranda e Pereiro. Les groupes de tresses du tore et de la bouteille de Klein. Tese de Doutorado, Caen, 2015. 6, 7, 10

[FN62] Edward Fadell e Lee Neuwirth. Configuration spaces. Mathematica Scandinavica, 10:111-118, 1962. 5

[GG17] Daciberg L Gonçalves e John Guaschi. Fixed points of n-valued maps on surfaces and the wecken property - a configuration space approach. Science China Mathematics, 60(9):1561-1574, 2017. 1, 2, 15, 17, 19, 20

[GG18] Daciberg L Gonçalves e John Guaschi. Fixed points of n-valued maps, the fixed point property and the case of surfaces - a braid approach. Indagationes Mathematicae, 29(1):91-124, 2018. 1, 2, 3, 16, 18, 19, 23, 55

[GGL19] Daciberg Lima Gonçalves, John Guaschi e Vinicius Casteluber Laass. The borsuk-ulam property for homotopy classes of self-maps of surfaces of euler characteristic zero. Journal of Fixed Point Theory and Applications, 21(2):65, 2019. 70

[GJ97] Daciberg Gonçalves e Jerzy Jezierski. Lefschetz coincidence formula on non-orientable manifolds. Fundamenta Mathematicae, 153(1):1-23, 1997. 2, 19

[GK06] Daciberg L Gonçalves e Micheal R Kelly. Wecken type problems for self-maps of the klein bottle. Fixed Point Theory and Applications, 2006(1):75848, 2006. 17, 20

[GK08] Daciberg L Gonçalves e Michael R Kelly. Coincidence properties for maps from the torus to the klein bottle. Chinese Annals of Mathematics, Series B, 29(4):425-440, 2008. 20

[Hat02] Allen Hatcher. Algebraic Topology. Cambridge University Press, 2002. 23

[Hop27] Heinz Hopf. Über mindestzahlen von fixpunkten. Mathematische Zeitschrift, 26(1):762774, 1927. 1 
[Joh97] David L Johnson. Presentations of groups. Number 15. Cambridge university press, 1997. 6

[KLY08] Hyun Jung Kim, Jong Bum Lee e Won Sok Yoo. Computation of the nielsen type numbers for maps on the klein bottle. J. Korean Math. Soc, 45(5):1483-1503, 2008. 17

[LS15] Roger C Lyndon e Paul E Schupp. Combinatorial group theory. Springer, 2015. 33, 48

[Mas67] William S Massey. Algebraic topology: an introduction. Springer New York, 1967. 16

[MKS04] Wilhelm Magnus, Abraham Karrass e Donald Solitar. Combinatorial group theory: Presentations of groups in terms of generators and relations. Courier Corporation, 2004. 33

[Sch84a] Helga Schirmer. Fix-finite approximation of n-valued multifunctions. Fundamenta Mathematicae, 1(121):73-80, 1984. 15, 16

[Sch84b] Helga Schirmer. An index and a nielsen number for n-valued multifunctions. Fundamenta Mathematicae, 124(3):207-219, 1984. 16, 17

[Sch85] Helga Schirmer. A minimum theorem for n-valued multifunctions. Fund. Math, 126(1):83-92, 1985. 1, 16

[Wec41] Franz Wecken. Fixpunktklassen. teil iii. mindestzahlen von fixpunkten. Mathematische Annalen, 118:544-577, 1941. 1 


\section{GRI Index}

Braid group pure braid group, 6 total braid group, 10

Classifying functions, 62 split, 24 